accepted by AJ April 23, 2006

\title{
Galaxies in the Hubble Ultra Deep Field: I. Detection, Multiband Photometry, Photometric Redshifts, and Morphology
}

\author{
Dan Coe ${ }^{1,2}$, Narciso Benítez ${ }^{1,2}$, Sebastián F. Sánchez ${ }^{3}$, Myungkook Jee ${ }^{1}$, Rychard \\ Bouwens $^{4}$, Holland Ford ${ }^{1}$ \\ coe@iaa.es, benitez@iaa.es, sanchez@caha.es, mkjee@pha.jhu.edu, \\ bouwens@ucolick.org, ford@pha.jhu.edu
}

\begin{abstract}
We present aperture-matched PSF-corrected $B V i^{\prime} z^{\prime} J H$ photometry and Bayesian photometric redshifts (BPZ) for objects detected in the Hubble Ultra Deep Field (UDF), 8,042 of which are detected at the 10- $\sigma$ level (e.g., $i^{\prime}<29.01$ or $z^{\prime}<28.43$ ). Most of our objects are defined identically to those in the public STScI catalogs, enabling straightforward object-by-object comparison. We have combined detections from $i^{\prime}, z^{\prime}, J+H$, and $B+V+i^{\prime}+z^{\prime}$ images into a single comprehensive segmentation map. Using a new program called SExSeg we are able to force this segmentation map into SExtractor for photometric analysis. The resulting photometry is corrected for the wider NIC3 PSFs using our ColorPro software. We also correct for the ACS $z^{\prime}$-band PSF halo. Offsets are applied to our NIC3 magnitudes, which are found to be too faint relative to the ACS fluxes. Based on BPZ SED fits to objects of known spectroscopic redshift, we derived corrections of $-0.30 \pm 0.03 \mathrm{mag}$ in $J$ and $-0.18 \pm 0.04 \mathrm{mag}$ in $H$. Our offsets appear to be supported by a recent recalibration of the UDF NIC3 images combined with non-linearity measured in NICMOS itself.

The UDF reveals a large population of faint blue galaxies (presumably young starbursts), bluer than those observed in the original Hubble Deep Fields (HDF).
\end{abstract}

\footnotetext{
${ }^{1}$ Johns Hopkins University, Dept. of Physics \& Astronomy, 3400 N. Charles St., Baltimore, MD 21218, USA

${ }^{2}$ Instituto de Astrofísica de Andalucía (CSIC), Camino Bajo de Huétor 50, Granada 18008, Spain

${ }^{3}$ Calar Alto Observatory, Almería E-040004, Spain

${ }^{4}$ University of California, Astronomy Dept., Santa Cruz, CA 95064
} 
To accommodate these galaxies, we have added two new starburst templates to the SED library used in previous BPZ papers. The resulting photometric redshifts are accurate to within $0.04\left(1+z_{\text {spec }}\right)$ out to $z<6$. Our BPZ results include a full redshift probability distribution for each galaxy. By adding these distributions, we obtain the redshift probability histogram for galaxies in the UDF. Median redshifts are also provided for different magnitude limited samples.

Finally, we measure galaxy morphology, including Sérsic index and asymmetry. Simulations allow us to quantify the reliability of our morphological results. Our full catalog along with our software packages SExSeg and ColorPro are available at http://adcam.pha.jhu.edu/ ${ }^{\text {coe/UDF/. }}$

Subject headings: cosmology: observations - galaxies: distances and redshifts - galaxies: evolution — galaxies: photometry — galaxies: statistics — galaxies: structure

\section{Introduction}

The Hubble Ultra Deep Field (UDF) provides us with our deepest view to date of the visible universe. It is located within one of the best studied areas of the sky: the Chandra Deep Field South (CDF-S). With a total of 544 orbits, it is one of the largest time allocations with HST, and indeed the filter coverage, depth, and exquisite quality of the UDF ACS and NICMOS images provide an unprecedented data set for galaxy evolution studies.

A comprehensive picture of galaxy formation and evolution must match the observed population statistics of integrated galaxy properties. These include the galaxy luminosity function, size distribution, and star formation rates all as functions of both redshift and environment. We must also be able to explain observed internal galactic structure, including bulge-to-disk ratio, asymmetry, and nuclear properties.

Large-area HST/ACS multiband surveys such as GEMS (Rix et al. 2004), GOODS (Giavalisco et al. 2004), and COSMOS (Scoville et al., in prep.) have contributed significantly to our understanding of galaxy evolution. These studies demonstrate the utility of high resolution multiband imaging. Multiband photometry allows robust determinations of photometric redshifts and even star formation rates, while high resolution imaging enables morphological classifications out to distant redshifts. The unparalleled depth and spatial resolution of the UDF dataset allow astronomers to extend studies like these to higher redshift.

To date, 76 spectroscopic redshifts have been obtained for galaxies within the UDF (see $\S 4.2 .1$ ), and more will surely be forthcoming. But, as was the case with the original Hubble 
Deep Fields (HDF-N Williams et al. 1996; HDF-S Williams et al. 1998), most of the objects detected in this field will elude spectroscopy for years to come. (We detect over 8,000 galaxies at $10-\sigma$ in the UDF.)

The original Hubble Deep Field (HDF-N) gave impetus to photometric redshifts, transforming the method from "A Poor Person's Z Machine" (Koo 1985) to the cosmological workhorse it is today. Spectroscopic redshifts are simply unattainable for about $95 \%$ of the objects in the HDF-N; these objects are too faint $(I \gtrsim 25)$, beyond the spectroscopic limits of today's telescopes. Steidel \& Hamilton (1992) had already demonstrated the powerful "dropout technique" for identifying high redshift galaxies based on rest frame Lyman- $\alpha$ absorption. And with the public availability of extremely high quality multi-band WFPC2 photometry (and subsequent near-IR observations from the ground), astronomers quickly refined the photometric redshift technique (from Gwyn \& Hartwick (1996) to Fernández-Soto et al. (1999, hereafter FLY99) and Benítez (2000)). Today, photometric redshifts are an essential tool for measuring galactic distances when spectroscopic redshifts are unavailable. In fact, high quality photometric redshifts based on multi-band photometry may be more robust than spectroscopic redshifts of low confidence (Fernández-Soto et al. 2001).

High quality photometry is the key to obtaining robust photometric redshifts. The UDF images are somewhat of a challenge in that respect, as the NICMOS images have wider PSF widths than the ACS images. If not handled properly, the measured NICMOS fluxes will be understated, by as much as 1 magnitude or more for small, faint objects. Our ColorPro software package enables us to obtain consistent aperture-matched and PSFcorrected photometry across all filters. The ACS $z^{\prime}$-band also sports a PSF halo which typically loses 0.1 magnitudes or more for faint objects. When properly accounted for, this extra $z^{\prime}$-band flux may provide a slight boost to measurements of star formation rate density at $z \sim 6$ (Paper II).

After obtaining robust $B V i^{\prime} z^{\prime} J H$ photometry, we use BPZ (Benítez et al. 2004) to obtain Bayesian photometric redshifts of the UDF galaxies. Spectral energy classifications are also obtained (e.g., elliptical, spiral, starburst). The Bayesian method not only yields more reliable photo-z's than traditional $\chi^{2}$ methods but also provides a measure of that reliability for each photo-z. In fact, BPZ returns an entire probability distribution $P(z)$ for each galaxy, which can then be summarized in terms of a most likely redshift and a confidence level and confidence interval for that redshift. The new version of BPZ takes the summary of $P(z)$ a step further by providing up to three high probability redshifts (the three highest peaks of $P(z)$ ) along with confidence levels and intervals for each. By adding the full redshift distributions $P(z)$, we obtain the redshift probability histogram for galaxies in the UDF. A markedly different (and less accurate) histogram emerges if one simply bins the single value 
best fit redshifts.

The main purpose of this paper is to present our method and catalog to the astronomical community. In $\S 2$ we describe the UDF observations. $\S 3$ describes our method for obtaining the photometric catalog. Our morphological measurements are described in $\S 3.5$. $\S 4$ presents our Bayesian photometric redshifts. And finally, we give a summary in $\S 5$. Our catalog and software are available at http://adcam.pha.jhu.edu/ coe/UDF/. In Paper II (Coe et al., in prep.) we examine the role of different galaxy types in the star formation history of the universe, as observed within the UDF.

\section{Observations}

The UDF (RA $=03^{h} 32^{m} 39$ s.0, Dec $=-27^{\circ} 47^{\prime} 29^{\prime}$. 1 (J2000)) was observed by the Wide Field Camera (WFC) of Hubble's Advanced Camera for Surveys (ACS, Ford et al. 2002) for a total of 400 orbits: 56 orbits each in the $B \& V$-bands (F435W \& F606W) and 144 orbits each in $i^{\prime} \& z^{\prime}$ (F775W \& F850LP) (P.I. Steven Beckwith $\left.{ }^{1}\right)$. These images cover $12.80 \operatorname{arcmin}^{2}$, over twice the area of each of the previous Hubble Deep Fields (HDF-N Williams et al. 1996; HDF-S Williams et al. 1998). We prune our catalog to the central $11.97 \mathrm{arcmin}^{2}$ of the ACS images, which has at least half the average depth of the whole image. The $B, V, \& i^{\prime} \mathrm{UDF}$ images are also $\sim 1.0,0.9$, \& 1.4 mags deeper than the respective HDF images. A filter similar to $z^{\prime}$ was not available to image the HDF, and its presence allows us to probe the UDF for $i^{\prime}$-band dropout galaxies at $5.7 \lesssim z \lesssim 7$.

For still higher redshift study, NICMOS's camera C3 "NIC3" was trained on this same patch of sky for an additional 144 orbits (P.I. Rodger Thompson ${ }^{2}$ ). While only covering $5.76 \operatorname{arcmin}^{2}$, or about half the ACS FOV, the NIC3 observations, split equally between the $J$ \& $H$-bands (F110W \& F160W), have the potential to reveal $z^{\prime}$-dropouts with redshifts $>$ 7. Transmission curves of the filters are shown in Fig. 1. Note that the filter we refer to as $J$ (or $J_{110}$ ) is actually much bluer than traditional ground-based $J$-band filters, fully overlapping the $z^{\prime}{ }_{850}$ filter and extending to $\lambda \sim 8000 \AA$.

See Tables 1 and 2 for a summary of the observations. The extinction corrections in Table 2 are derived from the dust maps of Schlegel et al. (1998), for which we obtained $E(B-V)=0.0079$.

\footnotetext{
${ }^{1}$ Director's Discretionary Cycle 12 Programs 9978 \& 10086: 9/24/03 - 1/16/04

${ }^{2}$ Cycle 12 Treasury Program 9803: 8/31/03 - 11/27/03
} 


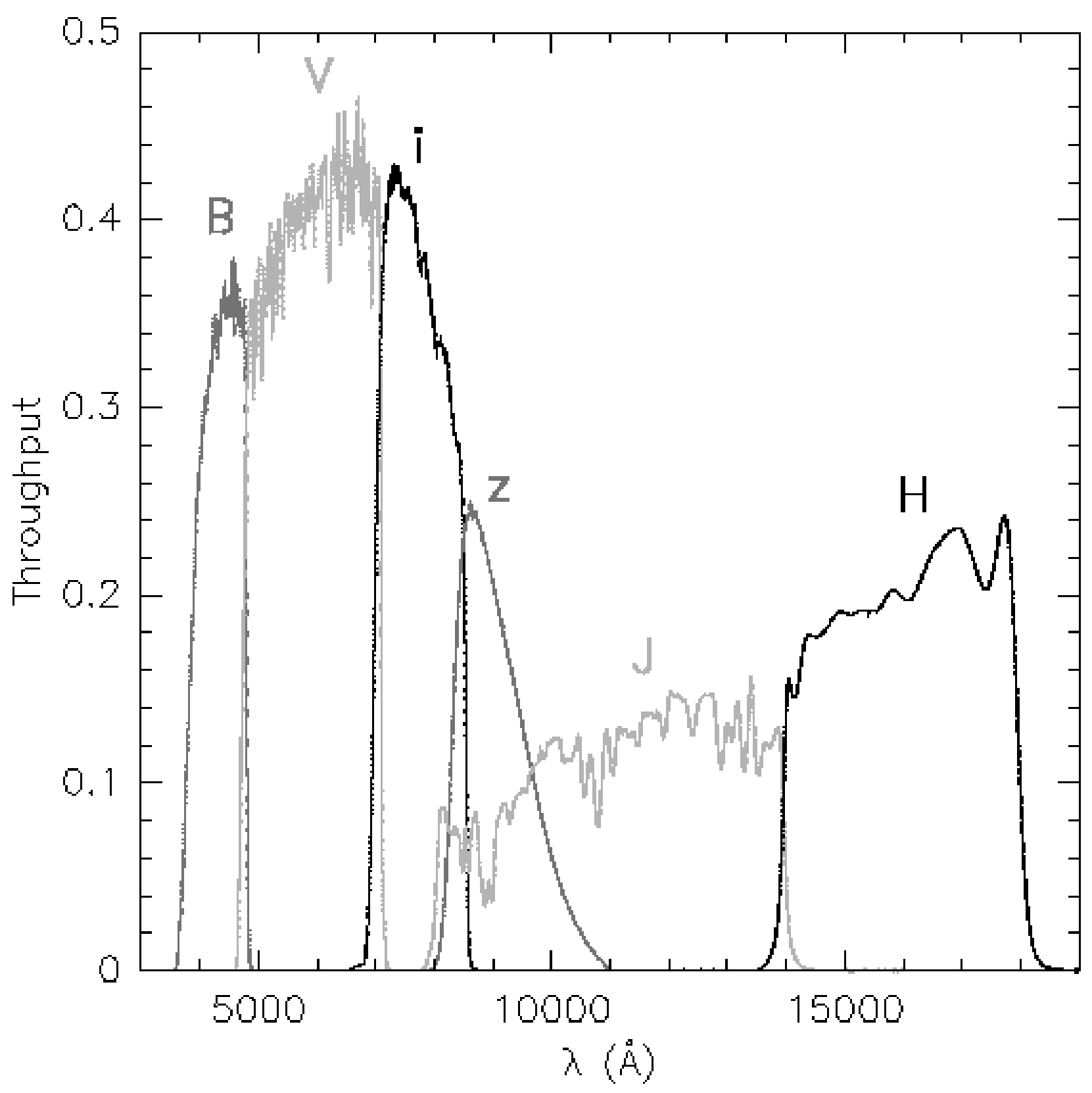

Fig. 1. - Transmission curves for the ACS $B V i^{\prime} z^{\prime} \&$ NIC3 $J H$ filters. The $i^{\prime} \& z^{\prime}$ filters are identical to those used on SDSS. The $J$ filter extends much further blueward than traditional ground-based $J$ filters. 
Table 1. UDF Imaging: Cameras

\begin{tabular}{cccc}
\hline \hline Camera/Detector & Resolution & Drizzled & Area \\
\hline ACS/WFC & $.05^{\prime \prime} / \mathrm{pix}$ & $.03^{\prime \prime} / \mathrm{pix}$ & $12.80 \mathrm{sq}^{\prime}$ \\
NICMOS/C3 ("NIC3") & $.20^{\prime \prime} / \mathrm{pix}$ & $.09^{\prime \prime} / \mathrm{pix}$ & $5.76 \mathrm{sq}^{\prime}$ \\
\hline
\end{tabular}

Table 2. UDF Imaging: Filters

\begin{tabular}{ccccccc}
\hline \hline Camera & Filter & Orbits & $\begin{array}{c}\text { Zeropoint }^{\mathrm{a}} \\
(\mathrm{AB})\end{array}$ & $\begin{array}{c}\text { Galactic }^{\mathrm{b}} \\
\text { Extinction }\end{array}$ & $\begin{array}{c}\text { Offset }^{\mathrm{c}} \\
\text { Depth }\end{array}$ \\
\hline ACS & $B(\mathrm{~F} 435 \mathrm{~W})$ & 56 & 25.673 & 0.0326 & $\ldots$ & 28.71 \\
$\mathrm{ACS}$ & $V(\mathrm{~F} 606 \mathrm{~W})$ & 56 & 26.486 & 0.0232 & $\ldots$ & 29.13 \\
ACS & $i^{\prime}(\mathrm{F} 775 \mathrm{~W})$ & 144 & 25.654 & 0.0160 & $\ldots$ & 29.01 \\
ACS & $z^{\prime}(\mathrm{F} 850 \mathrm{LP})$ & 144 & 24.862 & 0.0117 & $\ldots$ & 28.43 \\
NIC3 & $J(\mathrm{~F} 110 \mathrm{~W})$ & 72 & 23.4034 & 0.0071 & 0.30 & 28.30 \\
NIC3 & $H(\mathrm{~F} 160 \mathrm{~W})$ & 72 & 23.2146 & 0.0046 & 0.18 & 28.22 \\
\hline
\end{tabular}

aProvided in B04's wfc_README.txt and T04's NICMOS image headers.

${ }^{b}$ Subtracted from the zeropoints.

${ }^{\mathrm{c}}$ Empirically derived in $\S 4.2 .2$; subtracted from the NIC3 magnitudes.

${ }^{\mathrm{d}} 10-\sigma$ limiting $\mathrm{AB}$ magnitude within a $0.2 \mathrm{sq}^{\prime \prime}\left(0.5^{\prime \prime}\right.$ diameter $)$ aperture, after subtracting extinction and offsets. 
The ACS images were reduced at STScI by Beckwith et al. (2003, hereafter B04, where 2004 refers to the release date). The original images of $0.05^{\prime \prime}$ resolution were combined and drizzled Fruchter \& Hook (2002) to an even finer resolution of $0.03^{\prime \prime} /$ pixel. Pixel integrity was maintained by setting pixfrac $=0$. Meanwhile, the reduction of the NIC3 images was performed by Thompson et al. (2005). The original $0.20^{\prime \prime} /$ pixel images have been drizzled to $0.09^{\prime \prime}$ / pixel resolution. pixfrac was set to 0.6, which (as Thompson et al. point out) introduces correlation between neighboring pixels, and therefore artificially reduces the measured noise in the final NIC3 images. We use the method of Casertano et al. (2000) to restore the NIC3 noise maps to their true levels (see §3.3.2). The reduced images and noise maps are available to the public at http://www.stsci.edu/hst/udf.

Throughout this paper we use Thompson et al.'s version 1 NIC3 image reductions and catalog (hereafter T04). We have compared version 1 to two other reductions. Thompson et al. (2005) present version 2 featuring improved masking of bad pixels and slightly better alignment to the ACS images. To keep pace, we visually inspect objects detected in the version 1 NIC3 images and remove any obviously spurious sources. We also correct the slight version 1 alignment offset (§3.3). Otherwise, there are no magnitude offsets or other significant differences between version 1 and version 2. Meanwhile, Louis Bergeron has performed an independent reduction of the UDF NIC3 images (priv. comm.). This version yields objects between 0.04 and 0.08 magnitudes brighter in the $J$-band (based on analyses performed both by us and by Bahram Mobasher, priv. comm.). This issue appears to have been settled by a recent recalibration of the zeropoints of the Thompson et al. UDF images (Thompson et al. 2006). In $\S 3.4$ we discuss this recalibration as well as a count-rate dependent non-linearity that affects the calibration of all NICMOS images.

\section{Catalogs}

Along with the reduced images, B04 and T04 also released photometric catalogs at http://www.stsci.edu/hst/udf. The two catalogs were generated independently, one being based on the ACS images and the other being based on the NIC3 images. Thus, object detections and aperture definitions in each filter are in general inconsistent, and accurate ACS-NIC3 colors cannot be obtained from these catalogs (except perhaps for the brightest objects).

We have built our work upon the object detections performed by the two previous teams, in an effort to avoid an unnecessary proliferation of different catalogs with small differences among themselves. For most objects, our isophotal aperture definitions are identical to those used in the B04 catalog (given their "segmentation maps" (§3.1)). This allows direct 
comparison of our results on an object-by-object basis. To these objects we have added those detected in the T04 NIC3 segmentation map. And finally, we perform our own ACS and NIC3 detections, adding any "new" objects to complete our segmentation map.

Using a new program we have developed called SExSeg, we are able to force all of these object definitions into SExtractor (version 2.2.2; Bertin \& Arnouts 1996) for photometric analysis (§3.2). The resulting ACS \& NIC3 photometry has been obtained within consistent isophotal apertures in every filter. Isophotal apertures have been shown to produce the most robust colors, performing slightly better than circular apertures and much better than SExtractor's MAG_AUTO for faint objects (Benítez et al. 2004).

Our NIC3 photometry is also corrected to match the ACS PSF, yielding robust ACSNIC3 colors (§3.3). All photometry is performed on images in the highest resolution frame (the NIC3 images are remapped to the ACS frame). And photometry is performed on undegraded images whenever possible. Rather than degrade every image to the worst PSF, we only degrade our detection image enough to match the PSF of each individual filter.

Based on our BPZ fits to objects with known spectroscopic redshifts, we find disagreement between the ACS and NIC3 calibrations (§4.2.2). To correct for this, we apply simple offsets of $-0.30 \pm 0.03$ and $-0.18 \pm 0.04$ mag to the NIC3 $J$ and $H$-bands, respectively. The latest recalibration efforts (of the NIC3 images and of NICMOS itself) appear to support our derived offsets (§3.4).

Our detection and photometric catalogs are presented in Tables $3 \& 4$, respectively. These are also available as a single catalog which also includes the BPZ results. This catalog may be downloaded from http://adcam. pha. jhu.edu/ ${ }^{\sim}$ coe/UDF. Our ColorPro photometric software and SExSeg package are also available via this website.

Finally, our measurements of galaxy morphology are described in $\S 3.5$, and our morphological catalog is presented in Table 5. This catalog contains only those objects detected in B04's i'-band catalog. 
Table 3. Catalog: Detection

\begin{tabular}{lcrrrrrrrrrr}
\hline \hline ID $^{\mathrm{a}}$ altered $^{\mathrm{a}}$ & $\begin{array}{c}\Delta i_{S T}{ }^{\mathrm{b}} \\
(\mathrm{mag})\end{array}$ & $\begin{array}{r}\text { area } \\
(\mathrm{pix})\end{array}$ & \multicolumn{2}{c}{$\begin{array}{c}\text { RA \& DEC }(\mathrm{J} 2000) \\
(\text { degrees })\end{array}$} & $\begin{array}{c}x^{\mathrm{c}} \\
(\mathrm{pix})\end{array}$ & $\begin{array}{c}y^{\mathrm{c}} \\
(\mathrm{pix})\end{array}$ & wfcexp $^{\mathrm{d}}$ & sig $^{\mathrm{e}}$ & stel $_{\mathrm{i}^{\prime} \mathrm{f}}$ \\
\hline 1 & 0 & 0.0003 & 5693 & 53.16551208 & -27.82847977 & 4932.80 & 802.88 & 2.01 & 551.4 & 0.03 \\
$2^{*}$ & 1 & -0.3040 & 103 & 53.16449738 & -27.82928467 & 5040.27 & 706.25 & 1.84 & 13.4 & 0.00 \\
$3^{*}$ & 1 & -0.8914 & 76 & 53.16319275 & -27.82922173 & 5178.82 & 713.79 & 2.05 & 10.9 & 0.00 \\
4 & 0 & -0.0164 & 77 & 53.16295624 & -27.82913971 & 5203.87 & 723.62 & 2.06 & 10.1 & 0.17 \\
5 & 0 & 0.0010 & 269 & 53.16403580 & -27.82889175 & 5089.33 & 753.53 & 1.86 & 55.5 & 0.03 \\
\hline
\end{tabular}

Note. - Table 3 is published in its entirety in the electronic version of the Astronomical Journal. A portion is shown here for guidance regarding its form and content.

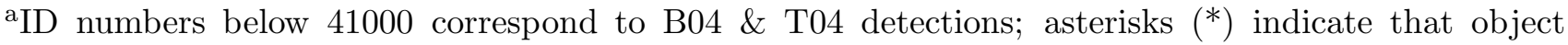
definitions have been altered (§3.1).

${ }^{\mathrm{b}}$ Rough guide to the degree of alteration: difference between our $i^{\prime}$-band magnitude and that from the B04 catalog.

${ }^{\circ}$ Coordinates in the B04 ACS images $\left(0.03^{\prime \prime} / \mathrm{pix}\right)$.

${ }^{\mathrm{d}}$ Exposure time in the ACS detection image $d$ normalized to the average depth of the whole image. For our analyses, we prune wf cexp $>0.5$.

${ }^{\mathrm{e}}$ Maximum detection significance from our 5 detections.

${ }^{\mathrm{f}}$ SExtractor stellarity measured in the $i^{\prime}$-band image. 
Table 4. Catalog: Photometry

\begin{tabular}{lrccccc}
\hline \hline ID & \multicolumn{1}{c}{$B_{435}$} & $V_{606}$ & $i_{775}^{\prime}$ & $z^{\prime}$ & $J_{110}$ & $H_{160}$ \\
\hline 1 & $24.10 \pm 0.01$ & $23.32 \pm 0.00$ & $22.80 \pm 0.00$ & $22.68 \pm 0.00$ & $-99.00 \pm 0.00$ & $-99.00 \pm 0.00$ \\
$2^{*}$ & $29.70 \pm 0.20$ & $29.26 \pm 0.10$ & $29.43 \pm 0.12$ & $29.12 \pm 0.17$ & $-99.00 \pm 0.00$ & $-99.00 \pm 0.00$ \\
$3^{*}$ & $29.60 \pm 0.17$ & $29.79 \pm 0.14$ & $30.14 \pm 0.20$ & $29.73 \pm 0.25$ & $-99.00 \pm 0.00$ & $-99.00 \pm 0.00$ \\
4 & $99.00 \pm 31.55$ & $29.56 \pm 0.12$ & $29.33 \pm 0.10$ & $29.34 \pm 0.18$ & $-99.00 \pm 0.00$ & $-99.00 \pm 0.00$ \\
5 & $28.04 \pm 0.07$ & $27.35 \pm 0.03$ & $26.93 \pm 0.02$ & $26.96 \pm 0.04$ & $-99.00 \pm 0.00$ & $-99.00 \pm 0.00$ \\
\hline
\end{tabular}

Note. - Table 4 is published in its entirety in the electronic version of the Astronomical Journal. A portion is shown here for guidance regarding its form and content. Magnitudes are "total" AB magnitudes with isophotal colors: NIC3 magnitudes are corrected to the PSF of the ACS images (§3.3). We have also applied offsets of $(J:-0.30 \pm 0.03, H:-0.18 \pm 0.04)$ to the NIC3 magnitudes (§4.2.2). Non-detections (listed, for example, as $99.00 \pm 31.55$ ) quote the 1- $\sigma$ detection limit of the aperture used on the given object. A value of -99.00 is entered for unobserved magnitudes: outside the NIC3 FOV or containing saturated or other bad pixels.

Table 5. Catalog: Morphology in the UDF $i^{\prime}$-band Image

\begin{tabular}{rccccrrrrr}
\hline \hline ID & $\chi^{2} / \nu$ & $\begin{array}{c}i^{\prime} 775 \\
(\mathrm{mag})\end{array}$ & $\begin{array}{c}R_{e} \\
(\text { pixels })\end{array}$ & \multicolumn{1}{c}{$a / b$} & \multicolumn{1}{c}{$\begin{array}{c}\theta \\
\text { (degrees) }\end{array}$} & $\begin{array}{c}n \\
\text { (Sérsic) }\end{array}$ & $\begin{array}{c}\text { dist } \\
\text { (pixels) }\end{array}$ & $\begin{array}{c}\text { Asym. } \\
\text { Index }\end{array}$ & $\begin{array}{c}\text { Number } \\
\text { Companions }\end{array}$ \\
\hline 1 & 1.835 & $22.72 \pm 0.01$ & $41.43 \pm 0.18$ & $0.13 \pm 0.00$ & $94.98 \pm 0.03$ & $1.28 \pm 0.01$ & 0.07 & 0.120 & 0 \\
2 & 1.098 & $29.34 \pm 0.10$ & $2.28 \pm 0.50$ & $0.71 \pm 0.21$ & $39.13 \pm 32.81$ & $0.5 \pm 0.75$ & 0.11 & 0.109 & 0 \\
3 & 1.143 & $30.22 \pm 0.37$ & $1.89 \pm 1.51$ & $1.99 \pm 1.94$ & $28.53 \pm 37.99$ & $0.80 \pm 1.98$ & 1.07 & 0.081 & 0 \\
4 & 1.198 & $29.37 \pm 0.33$ & $0.53 \pm 0.73$ & $0.12 \pm 1.13$ & $2.43 \pm 70.24$ & $3.98 \pm 11.78$ & 1.10 & 0.234 & 0 \\
5 & 1.220 & $26.88 \pm 0.02$ & $3.17 \pm 0.07$ & $0.55 \pm 0.02$ & $7.64 \pm 2.11$ & $0.67 \pm 0.07$ & 0.30 & 0.129 & 0 \\
\hline
\end{tabular}

Note. - Table 5 is published in its entirety in the electronic version of the Astronomical Journal. A portion is shown here for guidance regarding its form and content. Only galaxies in the B04 catalog are analyzed. ID numbers correspond to that catalog. The magnitude $i_{775}^{\prime}$, effective radius $R_{e}$, semiaxis ratio $a / b$, position angle $\theta$, Sérsic index $n$, and badness of fit $\chi^{2} / \nu$ are all derived from galfit. The distance between galfit's best fit centroid and that from B04 is given here as "dist". This distance is restricted to fewer than 2 pixels; dist $>1000$ indicates a misfit. The asymmetry index and number of companions are measured as described in §3.5. Additional columns in the electronic version are RA \& Dec (based on the B04 catalog) and galfit's best fit centroid $(x, y)$. 


\subsection{Synthesized $B V i^{\prime} z^{\prime} J H$ Detection}

Our catalog combines the results of five independent detections: two performed by B04 on the ACS image $\left(i^{\prime}, z^{\prime}\right)^{3}$, the T04 NIC3 detection $(J+H)$ and two performed by us $\left(B+V+i^{\prime}+z^{\prime}, J+H\right)$ (Table 6 and Fig. 2). Segmentation maps for the B04 and T04 detections were obtained from http://www.stsci.edu/hst/udf. Using their object definitions allows us to compare our photometry, photometric redshifts, etc. on an object-by-object basis, knowing that we have used identical apertures. ${ }^{4}$ Future groups may also wish to use these object definitions to facilitate comparison.

Our $B+V+i^{\prime}+z^{\prime}$ ACS detection image " $d$ " was created by dividing each image by the RMS of a "blank" region and then adding the four images. This allows the deepest possible detection in the ACS images for objects detected in all the filters. Similarly, we create a NIC3 $J+H$ detection image (like the one used by T04). We run SExtractor on these two images using the same parameters used by the UDF teams (to the best of our knowledge ${ }^{5}$, including the use of the ACS and NIC3 detection weight maps) producing our final two segmentation maps. Our NIC3 detection is slightly more aggressive than that performed by T04, yielding extra detections and larger isophotal apertures.

For each detection, SExtractor produces (upon request) a segmentation map. A segmentation map defines the pixels belonging to each object. It is an integer FITS image on the same scale as the detection image. Each pixel contains the ID number of the object it belongs to. If a pixel doesn't belong to an object, then it is set to zero. The segmentation map thus defines the location and extent of objects in the detection image (see Fig. 2).

\footnotetext{
${ }^{3}$ We neglect B04's "supplemental" $i$-band detection as neither the SExtractor parameters nor a segmentation map was readily available for this detection. However, we do serendipitously "re-discover" 5 of those 100 objects with our other detections. We reassign B04's IDs (in the 20000 range) to these objects. B04's 95 other "supplemental" objects are not found in our catalog; they remain blended with other segments.

${ }^{4}$ Using SExtractor alone, we were able to emulate B04's main i'-band catalog fairly well, but not exactly. Any attempt to reproduce another's catalog quickly becomes a lesson in SExtractor's sensitivity to input parameters. Beckwith et al. plan to publish their full set of input parameters in an upcoming paper. But we skirt the issue by applying their segmentation maps directly.

${ }^{5}$ For SExtractor detection of an object, the UDF teams require 9 contiguous pixels $0.61-\sigma$ above the background. The deblending parameters are DEBLEND_NTHRESH $=32$ and DEBLEND_MINCONT $=0.03$. And (at least for the NIC3 images), no global background is subtracted, but a local background is subtracted from each object, using an annulus of width BACKPHOTO_THICK $=24$.
} 


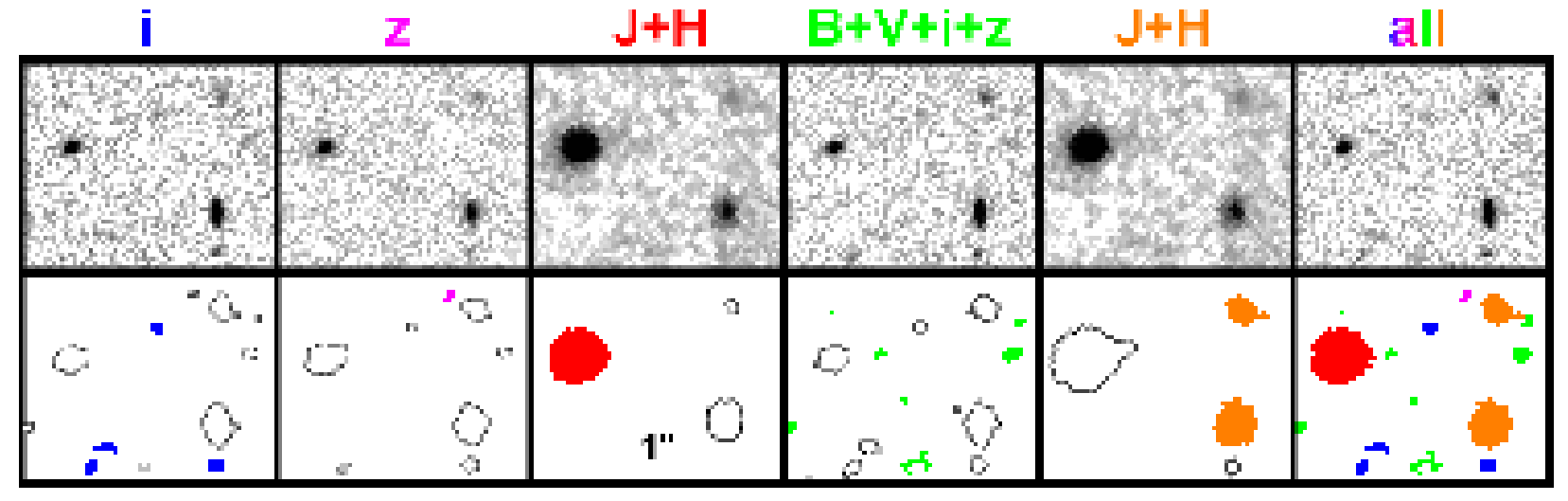

Fig. 2.- Comprehensive detection of faint objects demonstrated in a small region of the UDF. We begin with the $i^{\prime}$-band image (top left) and B04's corresponding segmentation map (bottom left) which defines their detections in that image. We then add "new" segments from four other detections. (The first three detections were performed by the B04 and T04 teams, and the final two (ACS $B+V+i^{\prime}+z^{\prime}$ and NIC3 $J+H$ ) are our own.) Some of these "new" objects are completely new, while others are simply re-definitions of objects previously detected to allow for larger apertures (see §3.1). The colored (filled) segments are the new segments in each detection that "survive" to the final comprehensive segmentation map (bottom right). This final segmentation map defines the photometric apertures that will be applied to all images. 
Table 6. Comprehensive Object Detection

\begin{tabular}{|c|c|c|c|c|c|}
\hline \multicolumn{2}{|c|}{ Detection } & \multirow{2}{*}{$\begin{array}{l}\text { Starting } \\
\text { ID }\end{array}$} & \multirow{2}{*}{$\begin{array}{c}\text { Objects } \\
\text { Detected }\end{array}$} & \multirow{2}{*}{$\begin{array}{c}\text { Objects in } \\
\text { Final Catalog }\end{array}$} & \multirow{2}{*}{$\begin{array}{c}\geq 10-\sigma \text { in } \\
\text { Final Catalog }\end{array}$} \\
\hline Filter & Author & & & & \\
\hline$i^{\prime}$ & B04 & $1^{a}$ & 10045 & 9989 & 6968 \\
\hline$z^{\prime}$ & B04 & $30001^{\mathrm{a}}$ & 7016 & 451 & 42 \\
\hline$J+H$ & T04 & $40001^{\mathrm{b}}$ & 926 & 6 & 6 \\
\hline$J+H$ & this paper & $41001^{\mathrm{c}}$ & 1414 & 71 & 28 \\
\hline$B+V+i^{\prime}+z^{\prime}$ & this paper & $50001^{\mathrm{c}}$ & 17692 & 8184 & 993 \\
\hline TOTAL & $\cdots$ & $\cdots$ & 37093 & $18706^{\mathrm{e}}$ & $8042^{\mathrm{e}}$ \\
\hline
\end{tabular}

${ }^{a}$ ID numbers below 40000 correspond to the B04 catalog. Segments that have been altered are flagged in our catalog and their ID numbers marked with an asterisk $(*)$ in this paper. However, most of the B04 objects (6,955 of their $i^{\prime}$-band detections) do retain their original definitions (segments).

${ }^{\mathrm{b}}$ We have added 40000 to the T04 ID numbers.

${ }^{c}$ The order of our final two detections is swapped in the catalog (cf. Fig. 2).

${ }^{d}$ Number of segments that survive more or less intact to our final catalog.

${ }^{\text {e}}$ The astute reader will have noticed that there are 5 extra objects in the total numbers. These correspond to objects in B04's supplemental $i^{\prime}$-band catalog that were serendipitously "discovered" and defined by our other detections. These objects retain their ID numbers (in the 20000 range) from B04's catalog. 
After remapping the NIC3 segmentation maps to the ACS frame, the five segmentation maps are combined using an automated procedure. ST's "main" $i^{\prime}$-band segmentation map serves as the starting point, and the other segmentation maps are compared to it: new segments are added and some old segments are enlarged (Fig. 2). To be more precise, a given segment is added if at least some fraction (we used $\frac{1}{3}$ ) of its pixels are "new" (don't already belong to an object). So not only are entirely new segments added, but we also add some segments that overlap with existing segments. "Disputed" pixels are always reassigned to the new segment. We are able to add any segment that overlaps just slightly with an existing segment. We also add any segment that is over 50\% larger than its predecessor. The old object is discarded whenever $\frac{2}{3}$ of its pixels have been consumed by the new object.

Replacing apertures with larger versions aids in obtaining robust photometry of dropout galaxies. If an object detected in the $i^{\prime}$-band image is brighter in $J+H$ and has a $(>50 \%)$ larger isophotal area in that image, then its larger $J+H$ segment will replace the "original" smaller $i^{\prime}$ segment. The larger segment takes advantage of the full $J+H$ signal. (Capturing the full signal is one of the reasons isophotal apertures outperform circular apertures, as mentioned in the introduction to this section $\S 3$. The smaller segment would not do the dropout galaxy justice, capturing only a fraction of its light in $J \& H$ and requiring a larger (and more uncertain) PSF correction (see Fig. 3).) Perhaps an even better strategy would be to enlarge apertures every time, regardless of how much larger the "new" segment is. Thus, a "maximal isophotal aperture" would be used for every object. We may explore this strategy in future work, but one of our goals for this paper was to maintain the integrity of objects defined in the catalogs released by STScI. ${ }^{6}$

The only drawback to enlarging objects in this way is that deblended objects are occasionally recombined. For example, if a $J+H$ aperture is $\geq \frac{1}{3}$ new it will be added to the segmentation map, regardless of the current segmentation in its footprint. Usually just one object (if any) will be supplanted. But occasionally multiple segments will be consumed (and thus united) by the new segment. (In the latest version of our software we do provide the option to forgo aperture enlargements in the event that multiple objects would be re-blended into one.) In the case of our catalog, 56 B04 $i^{\prime}$-band detections and $1 z^{\prime}$-band detection are thus consumed by neighboring objects. Of course perfect deblending was never the goal of this paper. Instead we are satisfied to base our catalog on the B04 and T04 detections, maintaining the majority of those definitions, while enlarging apertures and adding objects where deemed appropriate.

\footnotetext{
${ }^{6}$ SExSeg also gives us the ability to "correct" SExtractor's segmentation. We can actually redraw segments (to deblend objects, eliminate star spikes, etc.) and force SExtractor to analyze objects in the new corrected segments. We did not take advantage of this ability in this paper.
} 


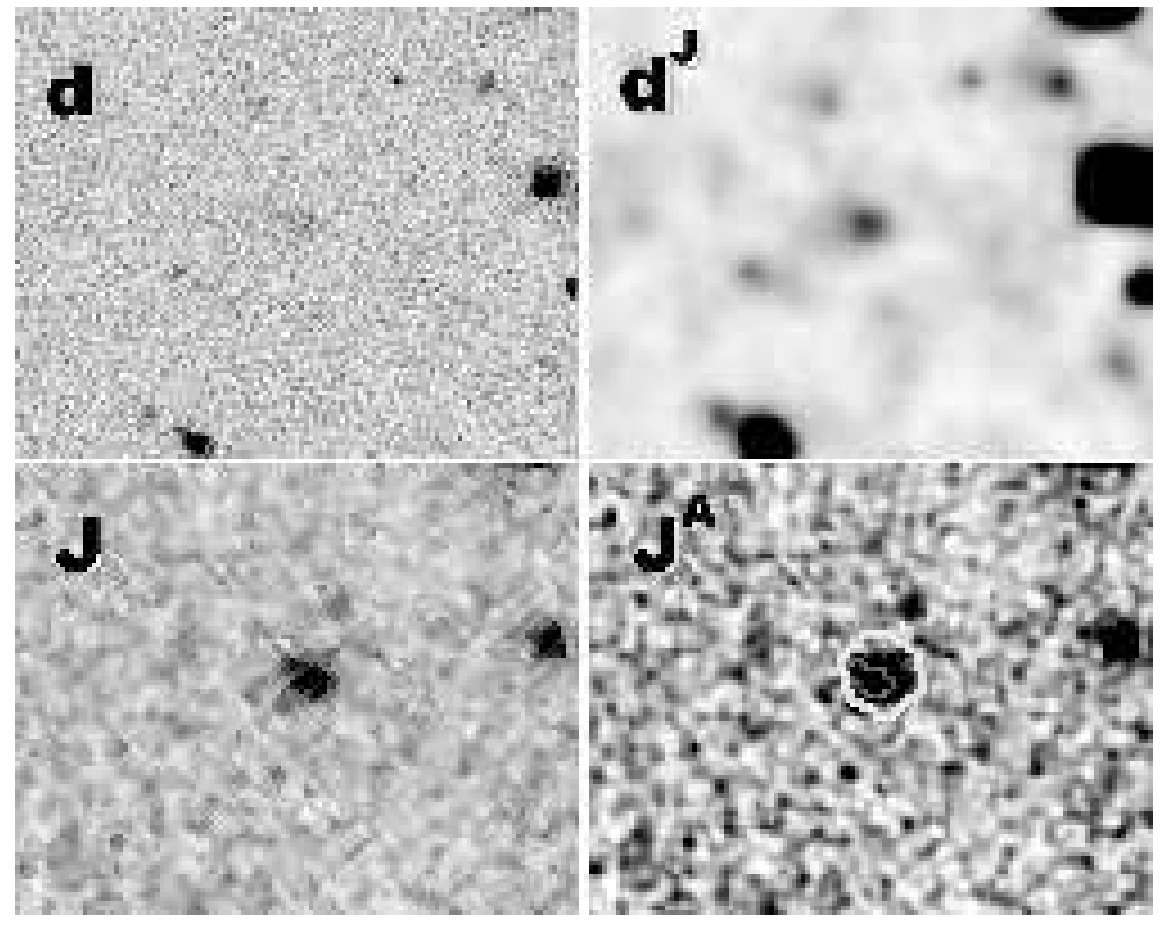

Fig. 3.- Four image stamps of the same region, centered on object \#1820*. This object was a faint detection in $d$, but it is much brighter in the NIC3 images. Top-left: the ACS detection image $\left(B+V+i^{\prime}+z^{\prime}\right)$. Top-right: the same image degraded to match the PSF of the NIC3 $J$-band image (bottom-left). Bottom-right: the $J$-band image re-mapped to the ACS frame and pixel scale; isophotal apertures are overlaid: the pink (inner) aperture was defined in $d$ while the yellow (outer) aperture was defined in the NIC3 detection image $J+H$ (and then re-mapped to the ACS frame). This object is significantly brighter in $J+H$ than in $d$. Thus its $J+H$ isophotal aperture is significantly larger than its isophotal aperture in $d$. The $d$ aperture is much smaller than the size of the object, requiring an unnecessarily large PSF correction. Our automated procedure replaces it with the larger $J+H$ aperture, taking advantage of the full signal for a more secure measurement of $d-J$. The asterisk $\left(^{*}\right)$ after the ID number indicates that the B04 segment for \#1820 has been altered, or replaced as in this case $(\S 3.1)$. (Despite the large color decrement $\left(z^{\prime}-J=1.55\right)$, object $\# 1820^{*}$ is probably not at high redshift. Its photometry is well fit by the SED of an elliptical galaxy at $z=1.98 \pm 0.35$.) It should be emphasized that this figure illustrates a rare occurrence. For most objects, the isophotal aperture is larger in $d$ than in $J$ because the ACS images are deeper than the NIC3 images. 
The final segmentation map is comprehensive, being formed by segments from the five independent detections (see Table 6). It defines the (isophotal) apertures that will be used for our photometric analysis of the ACS and NIC3 images, which we describe in the next subsection (§3.2). ID numbers in the segmentation map correspond to those from the B04 catalog, except in the cases of "new" objects (undetected by B04). These ID numbers are carried through to our catalog. Each B04 object is also flagged as to whether any alterations were made to the segment (whether any pixels were lost or the segment was replaced by a larger version). This flag takes the form of an asterisk $(*)$ appended to ID numbers in the text of this paper.

\subsubsection{New Galaxies}

We pause from describing our technique to consider what we have gained from our comprehensive object detection. Our automated procedure began with B04's i'-band segmentation map and added new objects from each of four other detections (§3.1, Table 6). Here we describe these new objects and the value they add to our investigation.

B04's $z^{\prime}$-band segmentation map adds 42 new objects detected at the 10- $\sigma$ level (Table 6 , last column). Upon visual inspection, most of these do appear to be legitimate $i^{\prime}$-band dropouts. And BPZ verifies that they probably lie beyond $z \gtrsim 6(\S 4.5)$. 4 of these objects appear to be spurious, while another 2 appear to be legitimate new objects now "de-blended" from larger B04 $i^{\prime}$-band segments.

T04's NIC3 $J+H$ segmentation map yields 6 "new objects" at 10- $\sigma$, including \#40819, the famous massive old $z \sim 6.5$ candidate galaxy, also known as HUDF-JD2 (Mobasher et al. 2005). It is for objects such as this that incorporation of T04's segmentation map is essential. Another potentially interesting object \#40925 fills in a very red patch amongst at least three other small galaxies. But \#40925 and its neighbors all appear to be at a redshift (or redshifts) of 2 or so. The other 4 "new objects" in this detection appear to be spurious: either spurious detections (from the glare of neighbors) or spurious re-segmentations. By "spurious re-segmentation", we mean that the object was previously detected, and now it is being re-detected slightly offset from the original. The new detection covers enough "new"

pixels to be added to our final segmentation map, but leaves enough $\left(>\frac{1}{3}\right)$ of the "old" segment uncovered that it survives as well (although missing a good chunk). These spurious re-segmentations could have perhaps been avoided with a tweaking of the $\frac{1}{3}$ parameter, or with a more sophisticated algorithm for combining segmentation maps. This proves to be a tricky business, akin to SExtractor's object de-blending. Our algorithm has room for improvement. But for now we allow for a handful of objects with poor segmentation out of 
a catalog of thousands.

In our own $J+H$ detection, we add 28 "objects" at 10- $\sigma$. Three of these (also featured in Table 10) don't correspond to optical detections, and if their NIC3 detections are confirmed could turn out to have very high redshifts indeed: \#41107 $\left(z_{b}=8.57_{-.83}^{+1.08}\right)$, \#41092 $\left(z_{b}=\right.$ $\left.7.73_{-.60}^{+1.31}\right)$, and \#41066 $\left(z_{b}=7.13_{-.54}^{+1.13}\right.$; with a faint $z^{\prime}$-band detection). The rest of our 28 detections appear to be spurious: a few new false detections, but mostly "spurious resegmentations", as discussed above. This occurs when the NIC3 segment is slightly offset from the ACS segment. The most likely explanation for this is that part of the galaxy appears brighter in the near-IR than the rest, which could be interesting in its own right. More exciting possibilities are that these are supernovae or other activity (between the time the ACS and NIC3 images were taken), or even chance alignments of galaxies slightly offset from more distant ones at very high redshift. But we will not be pursuing those possibilities here.

Finally, we discuss our $d=B+V+i^{\prime}+z^{\prime}$ detection, which is supposed to allow the deepest possible detection in the ACS images for objects detected in all the filters. Most of the 993 $10-\sigma$ objects in this detection are simply outside the field of view studied by B04. ${ }^{7}$ But the interesting ones are the 127 objects that we find inside B04's search area. Some are spurious re-segmentations, and there are a few wispy detections that are almost undoubtedly false. But many of these objects are faint blue galaxies, with $i^{\prime}$ and $z^{\prime}$-band fluxes too faint to be detected in these bands. Given the large population of faint blue galaxies visible in the UDF (see $§ 4.1$ ), it is important to include a detection such as this based (at least in part) on the bluer bands $B$ and $V$.

\subsection{SExSeg}

Armed with our single comprehensive segmentation map (the definition of objects and their extents), we need the ability to obtain multicolor photometry given these object definitions. To this end, we have developed a new program called SExSeg (part of the ColorPro package; Coe et al., in prep.), which forces SExtractor to run using a pre-defined segmentation map. We have chosen not to modify the SExtractor code itself, which although perhaps more straightforward, would involve changing a software which has become a de

\footnotetext{
${ }^{7}$ Those authors trimmed the edges of the ACS field to avoid regions of low signal to noise. Our catalog contains objects detected all the way out to the edge of the image. We only trim the edges as part of our analysis, and then we trim less area than B04. After trimming this detection, we're still left with 708 "new objects".
} 
facto standard and is well understood by many astronomers. Instead, SExSeg alters the input detection image based on the input segmentation map. When SExtractor is run on this new detection image it is forced to acknowledge the desired segments. SExtractor is then run in double-image mode with this new detection image and the desired photometric analysis image.

The input segmentation map is altered slightly by inserting gaps between neighboring objects. This ensures SExtractor's accurate and stable reproduction of the segmentation. Gaps are always created by discarding pixels from the larger of the two neighbors. And the number of pixels lost (if any) by each object is recorded in the catalog. But it must be emphasized that these slight segment alterations do not adversely affect our color measurements (Coe et al., in prep.), as we discuss below.

To demonstrate SExSeg's accuracy, we ran SExSeg on the original NIC3 images using the segmentation map provided by T04 (http://www.stsci.edu/hst/udf). We compare the resulting magnitudes to those given in the T04 catalog. For the majority of the objects, our magnitudes match T04's magnitudes exactly (Fig. 4). The only significant variations in magnitude arise from objects whose segments have been altered (where gaps were inserted between neighboring objects). These objects do get flagged in the catalog, but their colors should not be considered wrong or "off". The inserted gaps make our isophotal apertures slightly smaller than those used by T04 for these objects. But by consistently applying our apertures to all images (here $J \& H$ ), we ensure accurate color measurements. All of our $J-H$ color measurements match T04's measurements to within 0.1 mags (most match to within 0.01 mags). But where our color measurements disagree, we cannot say which method obtained the more accurate measurement. In other words, Thompson et al. can't say our method is "off" any more than we can say their method is "off". Simulations verify that SExSeg colors are just as accurate SExtractor colors (given the limits of photometric noise) when the segment has been altered (Coe et al., in prep.). Of course when the segment has not been altered (as is the case for the majority of objects in most images) the SExSeg colors are (almost always) identical to the SExtractor colors.

\subsection{Robust Aperture-Matched, PSF-corrected $B V i^{\prime} z^{\prime} J H$ Photometry}

Aperture-matched PSF-corrected photometry is essential to obtaining robust colors across images with varied PSF (see e.g. Benítez et al. 1999, Vanzella et al. 2001). Galaxy images blur as the PSF is degraded. The photometry of bright galaxies is not significantly affected, as we use large "maximal isophotal apertures" (§3.1). But for faint objects (with small isophotal apertures), the scant flux gets spread too thin, much of it getting swept 

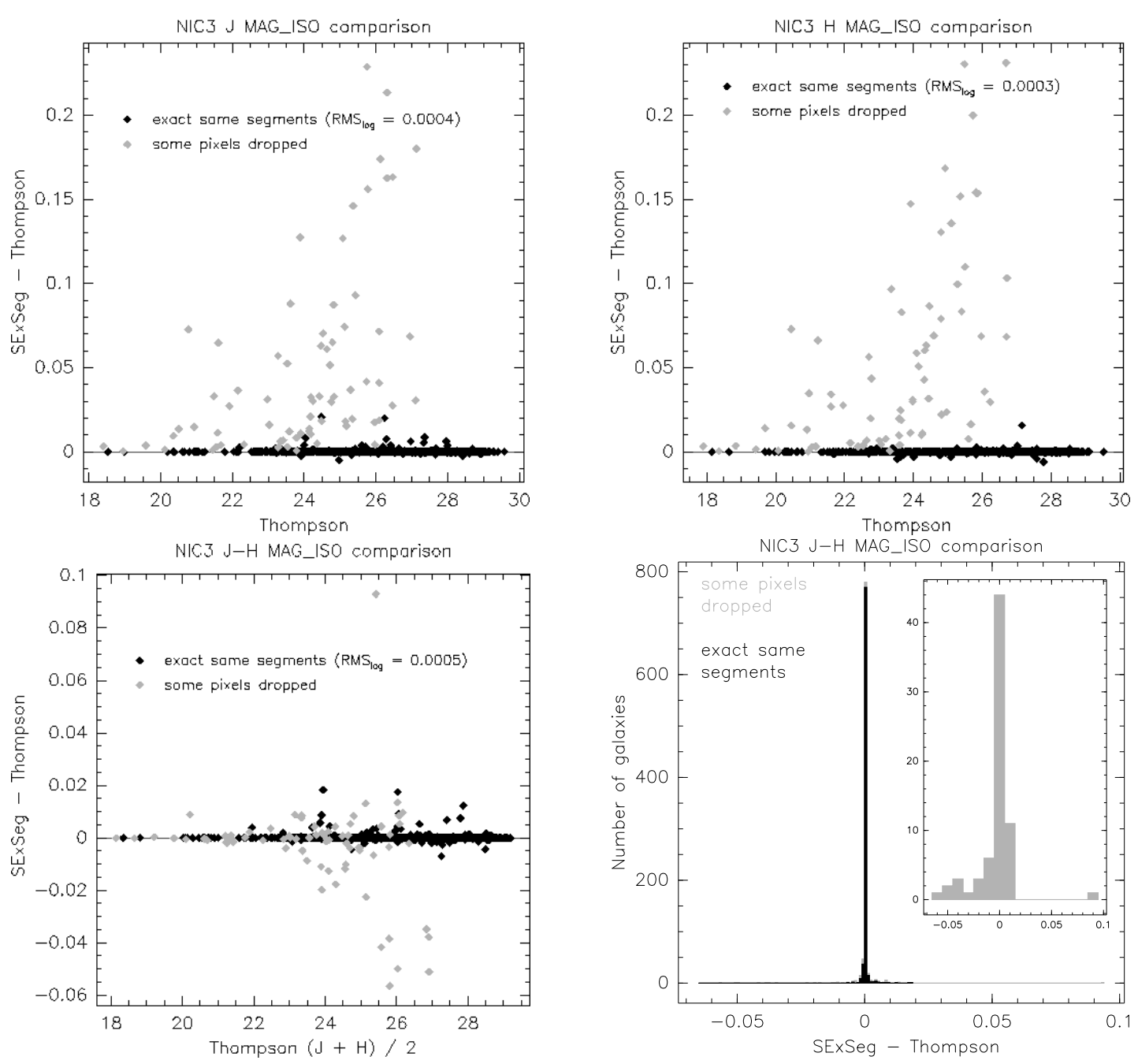

Fig. 4.- NIC3 SExSeg isophotal magnitudes and colors are compared to those derived directly from SExtractor by T04. SExSeg inserts gaps to separate neighboring objects; these altered segments are plotted in green. The lost pixels normally result in lost flux (higher magnitudes). However the main purpose of SExSeg is to measure accurate colors, and when apertures are slightly altered, they are still used consistently across filters. The resulting colors may be slightly different, but they are no less accurate given the effects of photometric noise (as verified by simulations, Coe et al., in prep.) Meanwhile, unaltered segments (black) usually yield identical magnitudes and colors (with occasional slight variations: logarithmic RMS values are on the order of $10^{-4}$ ). The histogram on the bottom right emphasizes that most objects have SExSeg $J-H$ colors identical to those measured by T04. 
under the "rug" that is the noise floor.

To estimate the flux loss, we degrade our best (ACS detection) image of the galaxy to the poor (NIC3) PSF and observe how much flux is lost. We then correct our observed NIC3 flux by the same amount (Fig. 5).

This procedure relies on the assumption that the ACS detection image is a good model for the NIC3 images. But what if a galaxy has a large internal color gradient? The ACS detection image is a stacked $B+V+i^{\prime}+z^{\prime}$ image. The resulting galaxy light profiles are the average of those in the four ACS filters. Thus they are less sensitive to internal color gradients. Also note that this is a non-issue for bright galaxies, for which the PSF corrections are small, regardless of internal color gradients.

We will now describe our process in more detail, as it is implemented in our ColorPro software.

The NIC3 $J$ image is mapped to the higher resolution ACS frame using IRAF's wregister ${ }^{8}$, taking care to preserve each object's flux by setting fluxconserve=yes and interp=spline3. The resulting image is referred to as $J^{A}$ (see Fig. 3). Next, we degrade the ACS detection image $d$ (the $B+V+i^{\prime}+z^{\prime}$ image) to the PSF of $J^{A}$, the result being $d^{J} .{ }^{9}$ For a given object, an identical aperture is used in $J^{A}, d$, and $d^{J}$, namely the isophotal aperture defined by the segmentation map via SExSeg (§3.2). Thus we measure magnitudes $J_{I S O}^{A}, d_{I S O}$, and $d_{I S O}^{J}$. The PSF correction is $d_{I S O}-d_{I S O}^{J}$, i.e. the difference in magnitudes resulting from the object being observed with the PSF of the NIC3 $J$-band as opposed to the PSF of ACS. This correction is applied to the $J$ magnitude yielding $J=J_{I S O}^{A}+\left(d_{I S O}-d_{I S O}^{J}\right)$. This PSF-corrected magnitude is the magnitude that would have been measured in the NIC3 image if it had the sharper ACS PSF. Thus this magnitude can be compared with magnitudes measured in the ACS filters, yielding robust colors $B-J, V-J, i^{\prime}-J$, and $z^{\prime}-J .{ }^{10}$ This process is repeated for the $H$-band image, which has a slightly worse PSF than $J$. It is important to note that the PSF corrections are different for every object. (This would be the case even if the same aperture size was used for every object.) And faint objects can have large PSF corrections of 2 magnitudes or more (see Fig. 6).

\footnotetext{
${ }^{8}$ The fits images released by ST contain accurate WCS information in their headers and thus aligned almost perfectly after wregister re-mapping. Perfect alignment was achieved by shifting the NIC3 WCS headers by a half pixel in both $\mathrm{x} \& \mathrm{y}$.

${ }^{9}$ This degradation must be performed carefully to avoid significant errors (of a magnitude or more) for faint objects. We discuss our robust procedure in the appendix.

${ }^{10}$ The $z^{\prime}$-band also requires a small PSF correction which we discuss in $§ 3.3 .1$.
} 


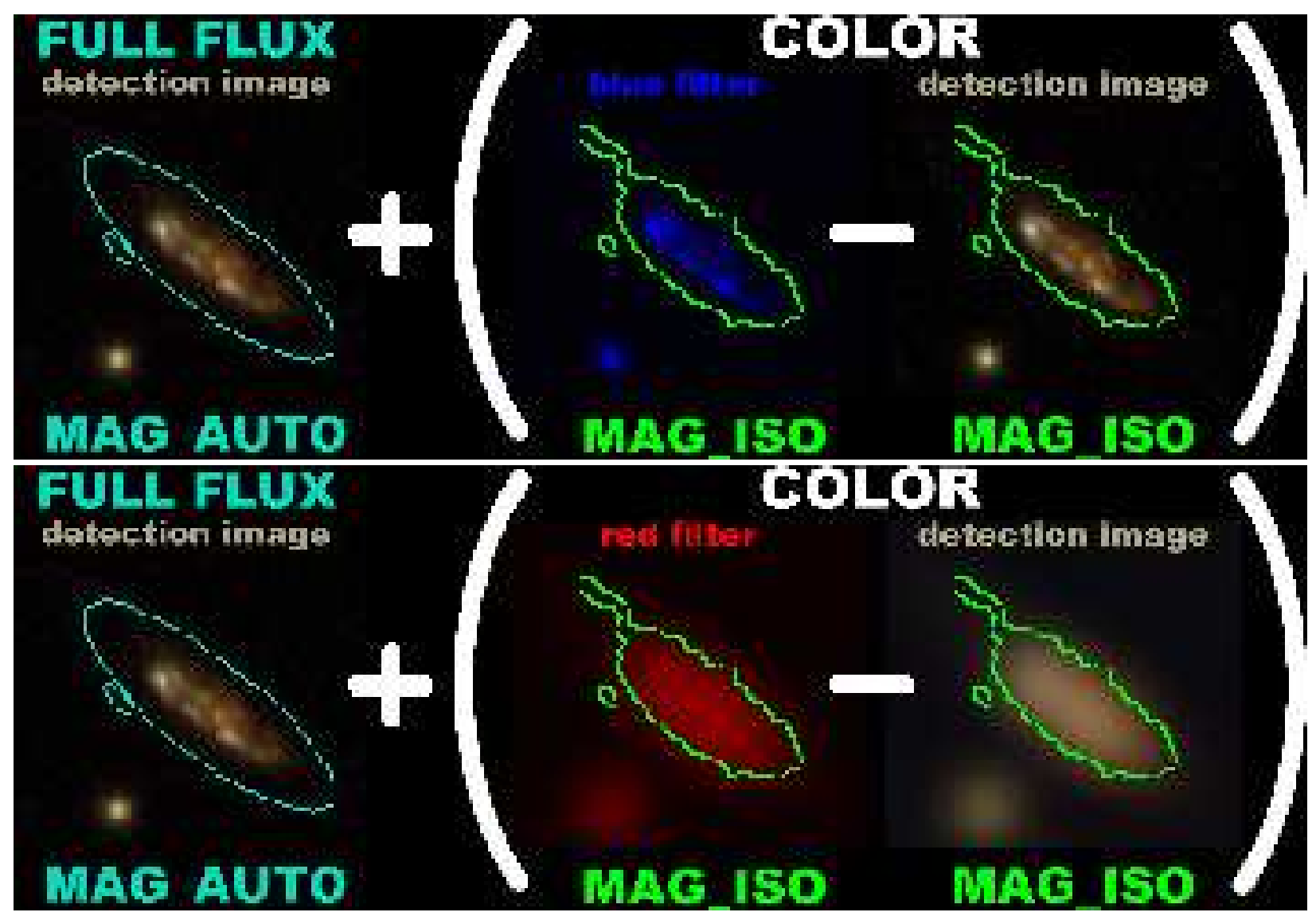

Fig. 5.- PSF-corrected isophotal aperture matched photometry. In the top frame, the blue magnitude $B=d_{A U T O}+\left(B_{I S O}-d_{I S O}\right)$, i.e. the total (MAG_AUTO) flux in the $B V i^{\prime} z^{\prime}$ detection image $d$ plus a color term measured within the object's isophotal aperture. In the bottom frame, we encounter a blurry red image. The red magnitude $J=d_{A U T O}+\left(J_{I S O}-d_{I S O}^{J}\right)$, where we have degraded our detection image to match the PSF of the blurry image. This PSF-corrected magnitude is the magnitude that would have been measured in the $J$-band image if it had the sharper $B$-band PSF. The resulting $B-J$ color measurement is robust. 
This procedure ensures consistent isophotal colors across all filters. But it is well known that isophotal magnitudes lose some flux; SExtractor's MAG_AUTO is a better measure of a galaxy's total flux (Bertin \& Arnouts 1996). So we obtain our final "total" magnitudes by applying a correction of $d_{A U T O}-d_{I S O}$ to each isophotal magnitude defined above. Rearranging terms, we have:

$$
\begin{aligned}
B & =\left(B_{I S O}-d_{I S O}\right)+d_{A U T O} \\
V & =\left(V_{I S O}-d_{I S O}\right)+d_{A U T O} \\
i^{\prime} & =\left(i_{I S O}^{\prime}-d_{I S O}\right)+d_{A U T O} \\
z^{\prime} & =\left(z^{\prime}{ }_{I S O}-d_{I S O}\right)+d_{A U T O}+z^{\prime} \text { apcor } \\
J & =\left(J_{I S O}^{A}-d_{I S O}^{J}\right)+d_{A U T O} \\
H & =\left(H_{I S O}^{A}-d_{I S O}^{H}\right)+d_{A U T O}
\end{aligned}
$$

Note that a given color across ACS filters is simply the isophotal color, e.g. $B-V=$ $B_{I S O}-V_{I S O}$ (except for the $z^{\prime}$-band, which requires its own PSF correction $z_{\text {apcor }}^{\prime}(\S 3.3 .1)$ ). But a color between ACS \& NIC3 filters contains the PSF correction term described above, e.g. $B-J=B_{I S O}-J_{I S O}^{A}+\left(d_{I S O}-d_{I S O}^{J}\right)$.

The above magnitude equations may look more familiar when reformulated as aperture corrections, for example:

$$
B=B_{I S O}-\left(d_{I S O}-d_{A U T O}\right)
$$

where we restore the flux lost as a result of using an isophotal aperture (assuming MAG_AUTO is our best measure of the total flux). But we prefer the previous set of equations, as they emphasize that every color is measured relative to the detection image $d$ in a consistent aperture, and that for each galaxy, $d_{A U T O}$ is just a constant added to each color.

Some objects lack measurements for $d_{A U T O}, d_{I S O}, d_{I S O}^{J}$, and/or $d_{I S O}^{H}$, either due to a total non-detection $(<1-\sigma)$ or perhaps saturation or other bad pixels. In these cases, we apply the average magnitude corrections successfully applied to other objects with those aperture areas (Fig. 6).

\subsection{1. $z^{\prime}$-band PSF Corrections}

ACS $z^{\prime}$-band images sport a slightly wider PSF than images in the bluer bands. Sirianni et al. (2003) have meticulously quantified the resulting PSF corrections as a function of both wavelength and aperture size. We use their results rather than relying on the degradation technique described above. 

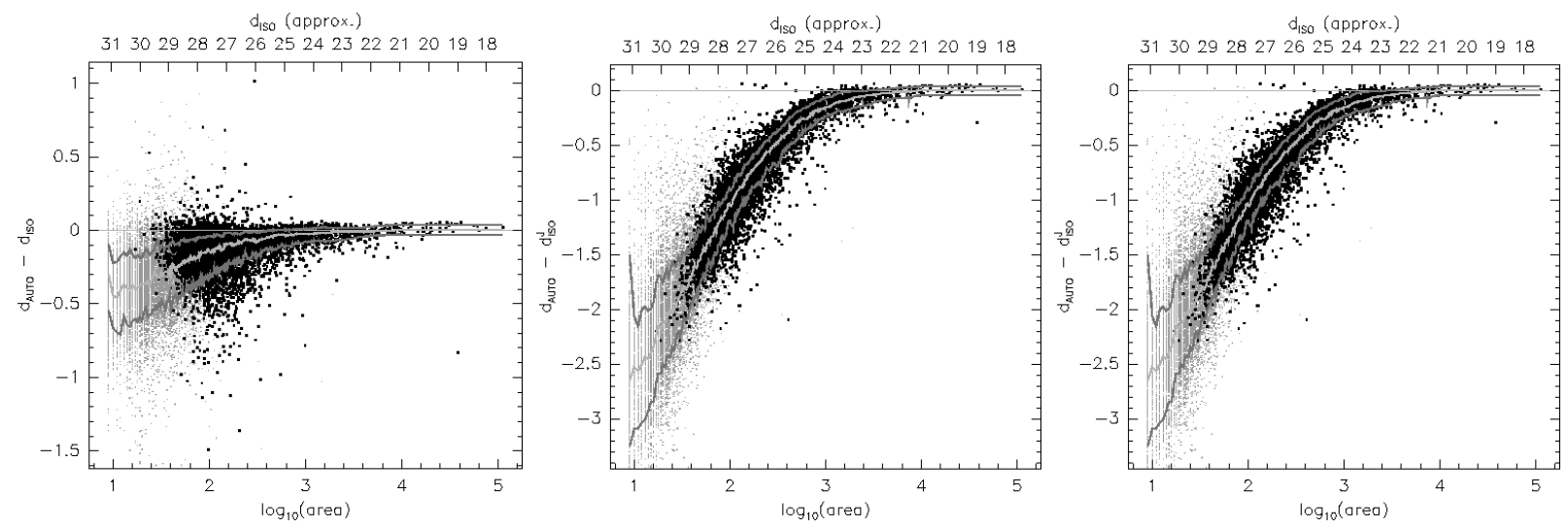

Fig. 6. - Left: Aperture corrections (MAG_AUTO - MAG_ISO) in the detection image $d$ plotted vs. isophotal aperture area in pixels. We take the liberty of labeling the top axis with approximate values for $d_{I S O}$, as isophotal magnitude and isophotal area are tightly correlated. The cyan line gives the median correction of each data point's 250 closest neighbors along the $\mathrm{x}$-axis. (Near the extrema in area, the number of neighbors is relaxed to as low as 100.) The magenta lines give the scatter $(1-\sigma)$ of these neighbors. All galaxies are included in this plot, but only those detected at 10- $\sigma$ are plotted in black. Lesser detections are plotted in grey, if at all (the y-axis does not extend to accommodate all of them). These $<10-\sigma$ detections do not significantly affect the average corrections, except to add more data points at low area. Center: aperture corrections in $d^{J}$ ( $d$ degraded to the PSF of $J$ ). Right: aperture corrections in $d^{H}$, to the same scale as the center plot. 
All ACS CCD detectors scatter light longward of $\sim 7500 \AA$ into a halo. The degree of scatter increases with wavelength. For a given galaxy observed in a given filter, we define the effective wavelength $\lambda_{\text {eff }}=\int d \lambda \lambda^{2} F_{\lambda}(\lambda) R(\lambda) / \int d \lambda \lambda F_{\lambda}(\lambda) R(\lambda)$, where $F_{\lambda}(\lambda)$ is the object's observed flux per unit wavelength, and $R(\lambda)$ is the response curve of the given filter (Fig. 1). Table 8 of Sirianni et al. (2003) provides aperture corrections (to infinite aperture size) as a function of aperture radius and effective wavelength $\lambda_{\text {eff }}$. These corrections are roughly independent of $\lambda_{\text {eff }}$ for observations in the $B, V$, and $i^{\prime}$ filters, but are much greater in the $z^{\prime}$-band. We subtract the $z^{\prime}$-band corrections from the $i^{\prime}$-band corrections (using a nominal value of $\lambda_{\text {eff }}=7750 \AA$ for the $i^{\prime}$-band), yielding the aperture corrections $z^{\prime}$ apcor that will bring our $z^{\prime}$-band magnitudes back in line with the other ACS filters. We plot these corrections in Fig. 7a for the expected range of $z^{\prime}$-band $\lambda_{\text {eff }}$ (Fig. 7b). Note that the aperture corrections are much smaller than those for the NIC3 filters.

Since we do not know a galaxy's $\lambda_{\text {eff }}$ until we assign an SED and redshift, we use the middle $9000 \AA$ curve as an initial guess, including an appropriate uncertainty: taking the top and bottom curves as our 95\% (2- $\sigma)$ confidence interval. Using this photometry, we run BPZ. Then, given each galaxy's SED and redshift, we re-calculate $\lambda_{\text {eff }}$ and thus $i^{\prime}-z^{\prime}$ for each galaxy. ${ }^{11}$ Finally, with our updated photometry, we re-run BPZ.

\subsubsection{Magnitude Uncertainties and Significance}

SExtractor calculates magnitude uncertainties using the weight maps released with the ACS images and the noise (RMS) maps released with the NIC3 images. The NIC3 noise maps were corrected for drizzling following Casertano et al. (2000). ${ }^{12}$ No such correction was necessary for the ACS images which were drizzled with pixfrac $=0$.

The NIC3 magnitude uncertainties must also account for the uncertainty of the PSF corrections. This uncertainty is difficult to measure directly, so we estimate it as the (1-

\footnotetext{
${ }^{11}$ This time, the uncertainties for $z^{\prime}$ apcor are the result of a Monte Carlo simulation: we reassign galaxy redshifts and SEDs given their BPZ probability distributions $P(z, t)$. Each realization yields values for $\lambda_{e f f}$ and thus $i^{\prime}-z^{\prime}$. The $1-\sigma$ scatter of these $i^{\prime}-z^{\prime}$ values (for each galaxy) give us our aperture correction uncertainty, which is added (in quadrature) to the $z^{\prime}$ magnitude uncertainty. These simulations were not carried out for galaxies detected at $<10-\sigma$. For these galaxies, we use the mean aperture correction uncertainties of 0.0086 for $z<5.7$ galaxies and 0.04 for $z>5.7$ galaxies.

${ }^{12}$ The NIC3 flux uncertainties are divided by $\sqrt{F_{A}}$ from Equation (A13) of Casertano et al. (2000). For $l>p, \sqrt{F_{A}}=1-p / 3 l$. For $p>l, \sqrt{F_{A}}=(l / p) \cdot(1-l / 3 p)$. For the NIC3 images, $p=$ pixfrac $=0.6$. For the object's linear size, we use $l=\sqrt{\text { area }}$, where area is the aperture size measured in input pixels (pre-drizzling: $0.20^{\prime \prime} /$ pixel).
} 

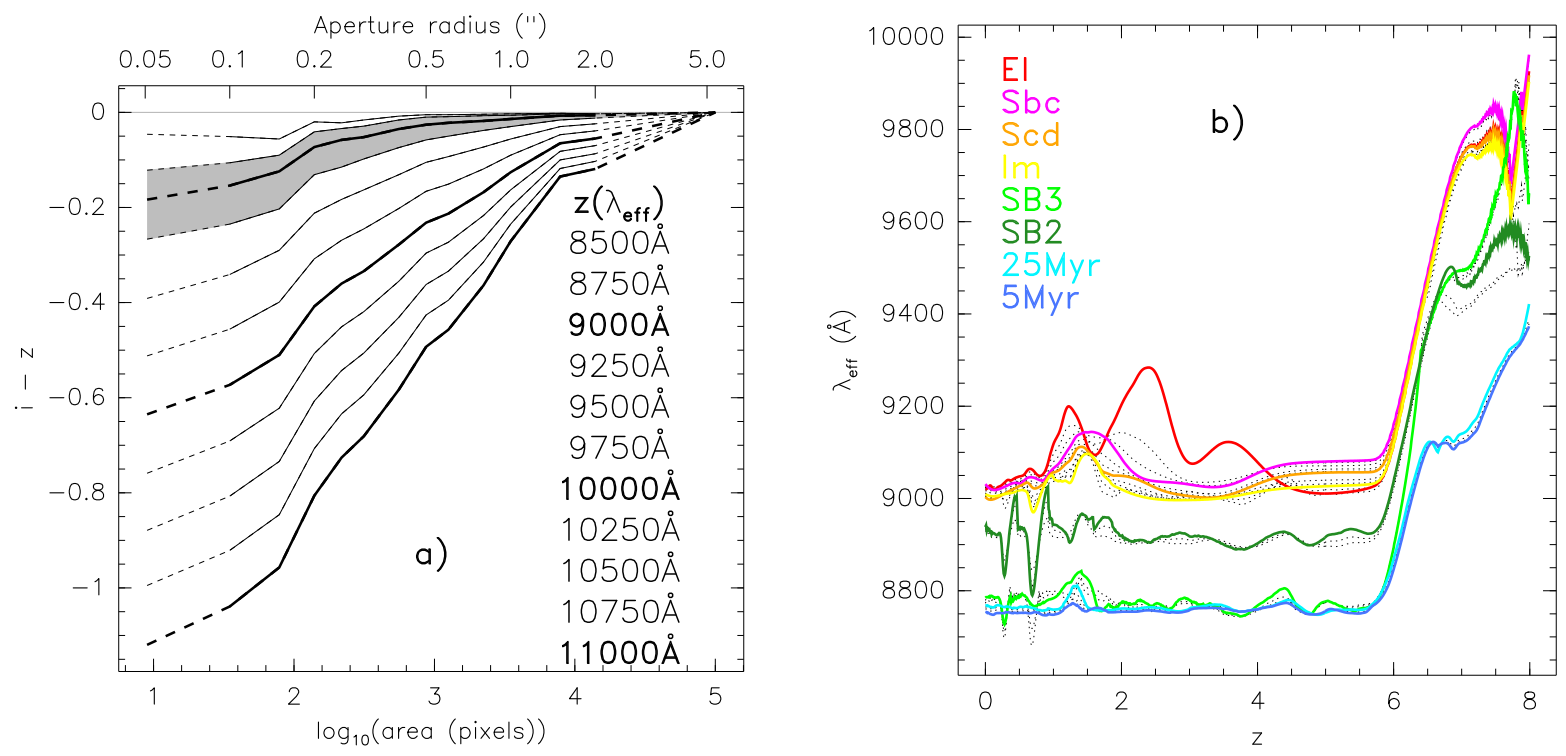

Fig. 7.- Left: Aperture corrections applied to the $z^{\prime}$-band photometry. The solid lines are taken from Table 8 of Sirianni et al. (2003). For a given object, the aperture correction depends on both the aperture radius (labeled across the top axis, with the corresponding area in the ACS images labeled across the bottom) and the effective wavelength $\lambda_{\text {eff }}$ of that object in the $z^{\prime}$-band (§3.3.1). Redder objects require larger aperture corrections. The dashed lines are extrapolations to smaller and larger radii. (To avoid negative aperture corrections, we simply assign zero aperture correction to $r=5.0^{\prime \prime}$.) The thicker lines merely indicate $\lambda_{\text {eff }}$ multiples of $1000 \AA$, while the shaded region is where most galaxies fall, as we see in our next plot. Right: Effective wavelength $\lambda_{\text {eff }}$ as a function of SED type $(\S 4.1)$ and redshift. The colors represent SED type, as in Fig. 11. Intermediate SED types are plotted as dotted lines. At $z \sim 5.7$, objects begin to drop out of the $z^{\prime}$-band, yielding significantly higher $\lambda_{\text {eff }}$. We assign no aperture correction to $z>8$ galaxies, as these have all but dropped out of the $z^{\prime}$-band, yielding meaningless $\lambda_{\text {eff }}$ and $i^{\prime}-z^{\prime}$. 
$\sigma)$ scatter of PSF corrections for a given aperture size (see Fig. 6). We then add this uncertainty in quadrature to the magnitude uncertainty reported by SExtractor. Also added in quadrature are uncertainties $(J: 0.025, H: 0.042)$ from our NIC3 magnitude offsets $(\S 4.2 .2)$.

As we are using isophotal apertures, we generally report isophotal magnitude uncertainties. However, some isophotal apertures are actually smaller than the PSF of the image (that is, a circle with a diameter of twice the FWHM of the PSF). Thus we also measure magnitude uncertainties within a circular aperture of each image's PSF size. We use FLUXERR_APER in place of FLUXERR_ISO whenever the isophotal aperture is smaller than the PSF. These area thresholds are 28 and 355 pixels $\left(0.03^{\prime \prime} /\right.$ pix), respectively for the ACS and NIC3 images.

We measure the significance of each detection in each filter as FLUX_ISO / FLUXERR (FLUXERR_ISO or FLUXERR_APER, depending on the aperture size). Most of our published results in $\S 4$ and Paper II employ a conservatively pruned catalog: any object without a $10-\sigma$ detection in any filter or detection image is discarded. Analysis of the inverted ACS detection image ( $d$ multiplied by -1$)$ yields 36 objects detected at the $10-\sigma$ level or higher. These are negative noise peaks, and we can expect to find a similar number of positive noise peaks (spurious objects) in our detection catalog. This is an insignificant level of contamination: $36 / 7,565=0.5 \%$. Even among the faintest of our pruned detections, between 10- and 11- $\sigma$, we only expect $3.5 \%$ to be spurious (599 objects vs. 21 found in the negative image, see also Fig. 8). Those interested may comb our full catalog for fainter sources. For example, the majority $(57 \%)$ of sources detected at 6 - to $7-\sigma$ will still be real.

A non-detection in any filter $(<1-\sigma$; FLUX_ISO $>$ FLUXERR $)$ is assigned a flux of zero and a flux uncertainty (upper limit) equal to the $1-\sigma$ detection limit. In table 2 , we quote $10-\sigma$ detection limits within a $0.5 \mathrm{sq}^{\prime \prime}$ aperture. The $1-\sigma$ limits are 2.5 magnitudes fainter. But our isophotal apertures vary greatly in size, and each aperture has a different detection limit. Fortunately, SExtractor custom-calculates a detection limit for each non-detection. This is given simply as FLUXERR (_ISO or _APER).

The upper flux limits assigned to NIC3 non-detections must incorporate PSF corrections. For example, FLUXERR may yield an upper limit corresponding to $J_{I S O}>29$ for a given aperture. But suppose this aperture has a $J$-band PSF correction of $d_{I S O}-d_{I S O}^{J}=-1$. Then an object just barely detectable in this aperture would see its magnitude corrected from $J_{I S O}=29$ to $J=28$. So a non-detection should be treated as $J>28$ when fitting SEDs to this object.

Finally, objects unobserved in a given filter (outside the NIC3 FOV or containing saturated or other bad pixels) are assigned infinite uncertainties. 


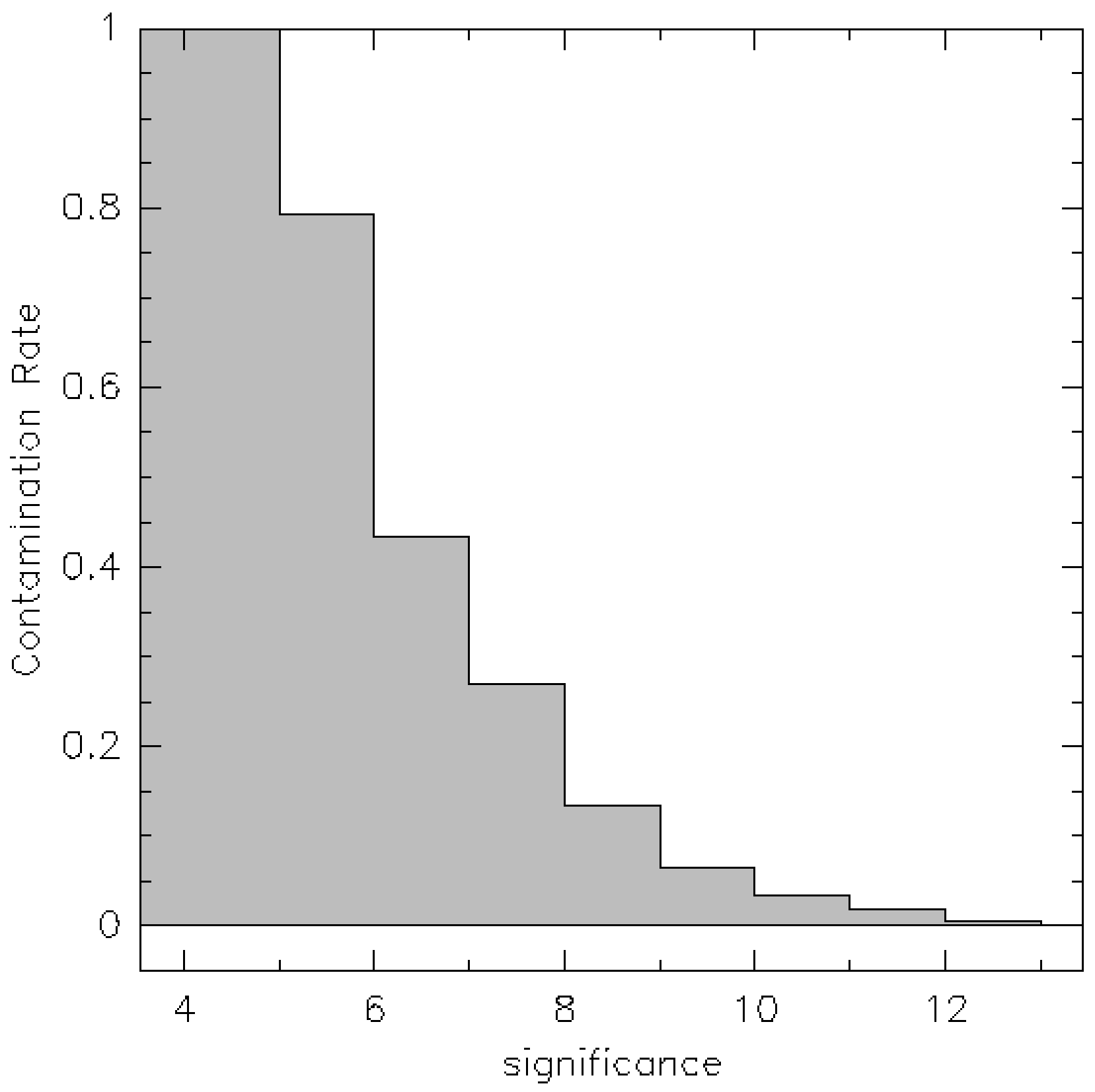

Fig. 8. - Spurious detection fraction in $d$ as a function of significance. For much of our analysis that follows, we prune our catalog at 10- $\sigma .599$ objects have been detected between 10 - and 11- $\sigma$ vs. 21 objects found in the negative image of $d$, yielding a $3.5 \%$ rate of contamination in that significance bin. Those interested in fainter sources may probe our catalog to as low as 6- $\sigma$. The majority (57\%) of sources detected at 6- to 7- $\sigma$ will still be real. 


\subsection{UDF NIC3 Recalibration}

Based on BPZ SED fits to objects of known spectroscopic redshift, we derived corrections of $-0.30 \pm 0.03 \mathrm{mag}$ in $J$ and $-0.18 \pm 0.04 \mathrm{mag}$ in $H$ (§4.2.2). Our derived corrections appear to be supported by two recent recalibrations: the first pertaining solely to the Thompson et al. UDF image reductions (both versions 1 \& 2) and the second affecting all NICMOS images. We discuss these recalibrations here.

Thompson et al. (2006) have recalibrated the zeropoints of their UDF images, resulting in objects brighter by $\sim 0.08$ and $\sim 0.09$ mag in $J$ and $H$, respectively. These offsets were due to $\mathrm{a} \sim 10 \%$ miscalibration of the filter sensitivity curves in their original analysis. Their catalogs (both versions $1 \& 2$ ) should be corrected for this recalibration (and that due to nonlinearity, as we discuss below). However, as the Thompson et al. images were not reduced by the standard STScI NICMOS pipeline, these offsets do not apply to any other (non-UDF) STScI NICMOS image reductions or catalogs. In fact, this correction brings the measured UDF NIC3 fluxes into better agreement with those measured internally and independently at STScI (Louis Bergeron, priv. comm.).

Meanwhile, STScI has been investigating issues of NICMOS non-linearity dependent on count rate (de Jong et al. 2006b). (This is not to be confused with the non-linearity inherent in all IR detectors which is dependent on total counts. This effect is well understood and corrected for in the NICMOS pipeline.) Apparently, brighter objects (with higher count rates) register slightly higher total fluxes than expected in NICMOS images, while fainter objects register slightly lower fluxes than expected. This effect was first discovered by Bohlin et al. (2005), followed up (Bohlin et al. 2006), and recently confirmed by robust lamp on/off tests (de Jong et al. 2006a). The results from this latter report show that for each dex (2.5 mag) decrease in incident flux, NIC3-observed $J$-band magnitudes drop $\sim 0.048$ more than expected. $H$-band magnitudes suffer a similar but weaker non-linearity of $\sim 0.016 \mathrm{mag} /$ dex. This presumably applies to all NICMOS images.

The UDF NIC3 images were calibrated relative to standard stars of $\sim 12^{\text {th }}$ mag which is $\sim 4$ dex (10 mag) brighter than the sky-background of the UDF. Thus sky-dominated objects in the UDF are expected to suffer offsets of $\sim 0.19 \mathrm{mag}$ in $J$ and $\sim 0.06 \mathrm{mag}$ in $H$ due to this count-rate dependent non-linearity. (By sky-dominated objects, we mean those objects with count rates less than that of the sky background. The total count rate of these objects (galaxy + sky) is therefore roughly equal to that of the sky itself.) For brighter UDF objects the offsets should be slightly less, decreasing by $\sim 0.048$ and $\sim 0.016 \mathrm{mag} / \mathrm{dex}$, respectively

for $J$ and $H$. Objects with $J \sim 22$ or $H \sim 22$ have roughly the same count rates as the sky in that filter, yielding total count rates $\sim 2 \times$ that of the sky. Thus the offset for a $J \sim 22$ object decreases slightly to $0.19-0.01=0.18\left(\right.$ where $\left.0.01 \sim 0.048 \times \log _{10}(2)\right)$. And an object 
1 dex fainter than that at $J \sim 19.5$ would have an offset of roughly $0.18-0.048=0.13$. But $J \sim 19.5$ objects are very rare in the UDF. Only 5 objects are brighter than $J<19.5$ with none brighter than $J<18$. In fact there are only 38 objects brighter than $J<22$. Thus to correct for this non-linearity, a constant offset of 0.19 mag in $J$ should prove an excellent approximation, especially for those $2,800+$ other objects detectable in $J$ but fainter than $J>22$. Similarly, a constant 0.06 mag offset should adequately correct the $H$ band magnitudes.

Proper corrections for non-linearity require corrections on a pixel-by-pixel basis, which will be implemented into a future version of the STScI NICMOS pipeline. As of April 2006, a beta version of software capable of performing this correction on NICMOS images was made available to the public. ${ }^{13}$ When run on the UDF, this software yields magnitude offsets similar to those quoted above, although small uncertainties still remain, pending further calibration tests (de Jong 2006).

When the magnitude offsets due to non-linearity are added to those due to the filter recalibrations described above, we find total offsets of $\sim 0.27$ and $\sim 0.15$ mag in $J$ and $H$, respectively. Thus, given the UDF NIC3 images with their original zeropoints, a $J=24$, $H=24$ object would be observed to have $J \sim 24.27$ and $H \sim 24.15$. Note that these offsets are very similar to those we quoted above, as derived empirically in $\S 4.2 .2$ from SED fitting using BPZ (based on the assumption that the ACS photometry was accurate). Thus we are encouraged to proceed with our analysis given our derived offsets: $-0.30 \pm 0.03$ in $J$ and $-0.18 \pm 0.04$ in $H$. (The uncertainties are added in quadrature to each object's NIC3 magnitude uncertainties.)

\subsection{Morphology}

To increase the utility of our catalog, we have included measures of several morphological parameters that are useful in automatic galaxy classification. These include Sérsic (1968) index $n$, asymmetry, and number of nearby neighbors.

For isolated and undisturbed galaxies, the Sérsic index $n$ alone is a fairly reliable indicator of morphological type (e.g., Andredakis et al. 1995). We adopt $n=2.5$ as the dividing line between disk- $(n<2.5)$ and spheroidal-dominated $(n>2.5)$ galaxies ("late" and "early" type, respectively), consistent with the analysis conducted by the Sloan Digital Sky Survey (SDSS; see Shen et al. 2003), and more recently the Galaxy Evolution by Morphology and

\footnotetext{
${ }^{13}$ http://www.stsci.edu/hst/nicmos/performance/anomalies/nonlinearity.html
} 
SEDs (GEMS) Survey (Rix et al. 2004). Simulations (§B) indicate that 80-95\% of galaxies in our catalog with $\sigma n / n<1$ (confident measures of $n$ ) have a correct morphological classification (late vs. early type, assuming that $n=2.5$ is a perfect discriminator). And this cut only discards $\sim 8 \%$ of the catalog.

Less well behaved galaxies, including mergers and irregulars, generally do not have well defined Sérsic indices. Fortunately these galaxies can generally be weeded out (or selected for) by measuring their large asymmetries (e.g., Conselice et al. 2003). Meanwhile, neighbors in projection can also affect the model fitting (stymieing even the most careful attempts to mask the neighbors out). Thus, in our catalog we also provide counts of nearby neighbors, which may be used to select well isolated galaxies, or alternatively, to help find interacting galaxies. Any reliable morphological classification should take all three parameters into account: Sérsic index, asymmetry, and nearby neighbors.

All of our morphological measurements are obtained from the $i^{\prime}$-band image (the deepest ACS image). We analyze every object in B04's $i^{\prime}$-band catalog ${ }^{14}$, beginning with the brightest galaxy and working our way down to the faintest. Along the way we subtract each galaxy model from the $i^{\prime}$-band image (see Fig. 9).

Thus we begin by creating a postage stamp, $5 \times r_{50}$ on a side, for the brightest galaxy, where $r_{50}$ is the galaxy's half-light radius, as given by SExtractor. Within that postage stamp, neighboring galaxies are masked out using ellipses, each ellipse given a minor axis length $b=2 \times r_{50}$ for that galaxy. (Note that we do not use our segmentation map (§3.1) to measure morphological parameters. We have not studied the effects of segmentation on such measurements, and thus we opt for a more traditional approach.) Using galfit (Peng et al. 2002), the brightest galaxy is fit to a single component Sérsic model $\Sigma(r) \propto$ $\exp \left(-\kappa_{n}\left[\left(R / R_{e}\right)^{1 / n}-1\right]\right)$, where $\kappa=\kappa(n)$ is a normalization constant and $R_{e}$ is the effective radius. The fit is constrained to $0.2<n<8$ and $0.3<R_{e}<500$ pixels, and the centroid is confined to within 2 pixels of the position derived by SExtractor. As initial guesses for the galfit parameters, we use the SExtractor output parameters given in B04's i'-band catalog. Lacking estimates for the Sérsic index from SExtractor, we start all fits with $n=1.5$.

Having been calculated for the brightest galaxy, the Sérsic model is subtracted from the $i^{\prime}$-band image. This subtraction benefits the subsequent modelling of all fainter nearby galaxies. We proceed to model the second-brightest galaxy, and continue in order of decreasing brightness, modelling and subtracting every galaxy in B04's i'-band catalog. Of the

\footnotetext{
${ }^{14}$ The relationship between our catalog and the ST catalog is well defined, with most objects being defined identically $(\S 3.1)$.
} 


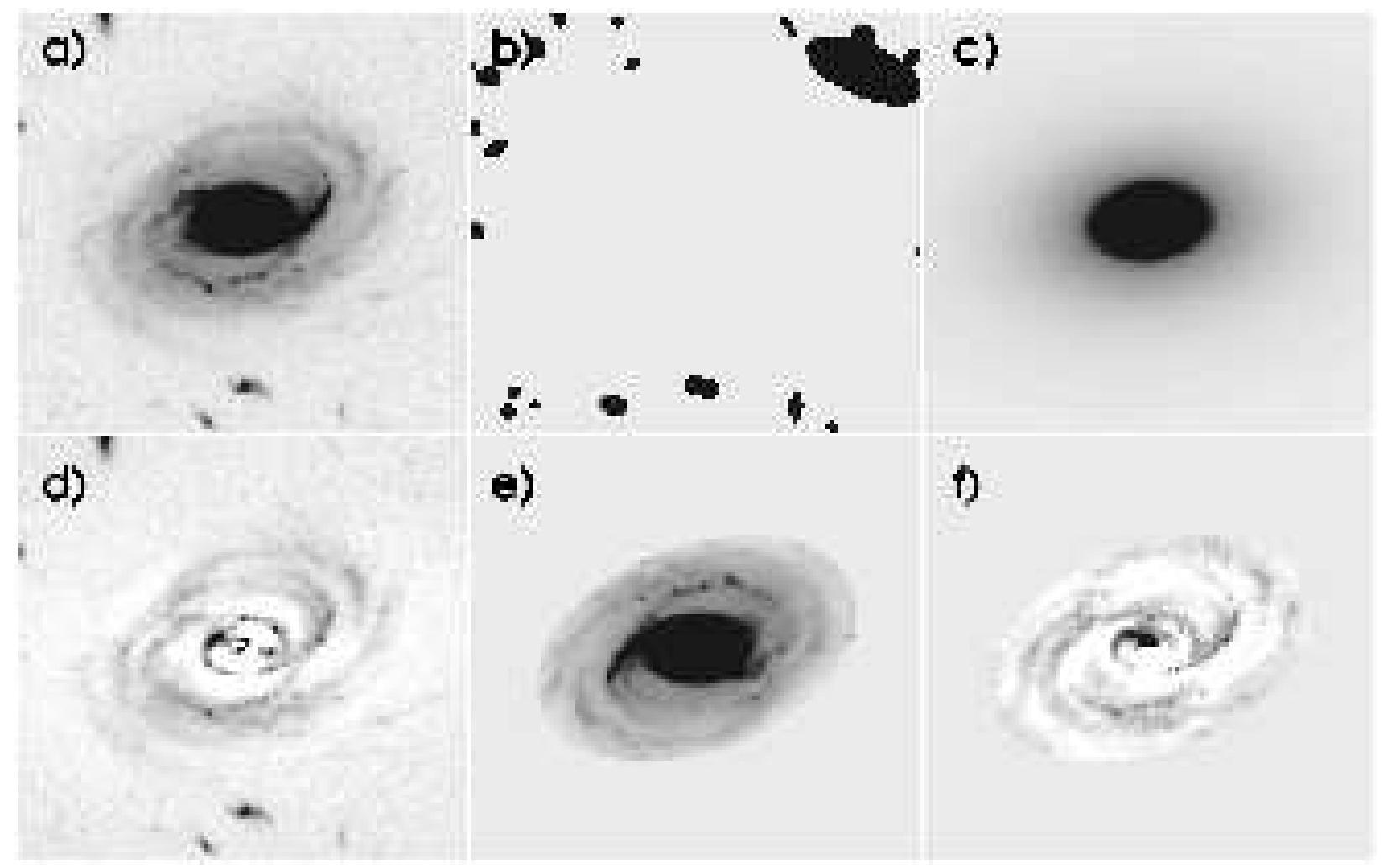

Fig. 9.- Recursive procedure used to obtain morphological measurements. (a): $i^{\prime}$-band image. (b): Ellipses used to mask out neighbors from the model fitting. $(c)$ : Resulting single component Sérsic model from galfit. $(d)$ : Model subtracted from $i^{\prime}$-band image. This galaxy will "remain" subtracted for the subsequent modelling of all fainter galaxies. $(e)$ : Galaxy rotated by $180^{\circ}$, and framed within an ellipse of $b=4 \times r_{50}$. $(f)$ : Difference of (a) and (e), used to measure galaxy asymmetry. This spiral galaxy shows a fair amount of asymmetry, but not enough to be flagged as "Irregular" or a merger (see Fig. 10). 
9,339 objects with stellarity $<0.9$, galfit derives meaningful output for 8,805 , or about $94 \%$ of the objects. Table 5 summarizes the resulting fit parameters and their uncertainties: magnitude $i^{\prime}$, effective radius $R_{e}$, ellipticity $a / b$, position angle $\theta$, and Sérsic index $n$. We also give the "badness" of each fit $\chi^{2} / \nu$.

Examples of early type $(n>2.5)$, late type $(n<2.5)$, and highly asymmetrical galaxies are given in Fig. 10. For the latter, Sérsic fits often prove unreliable, as mentioned above. Thus we measure asymmetry:

$$
A=\frac{\Sigma\left|I_{i, j}-I_{i, j}^{r o t}\right|}{2 \Sigma\left|I_{i, j}\right|}
$$

where $I_{i, j}$ are the pixel values and $I_{i, j}^{r o t}$ is the image rotated by $180^{\circ}$ (Schade et al. 1995; Abraham et al. 1996; Conselice et al. 2000). These measurements are obtained within an ellipse of $b=4 \times r_{50}$ drawn around the galaxy (with neighbors masked out and brighter galaxies subtracted as above, Fig. 9e). This index proves to be a good estimate of asymmetry for galaxy images with good signal-to-noise (Conselice et al. 2000). Our method does not minimize the asymmetry, and in that respect it is slightly different from the method of Conselice et al. (2000).

For each galaxy, we also give the number of nearby neighbors, or companions. Two galaxies are identified as companions if their centroids lie within twice the sum of their effective radii and their $i^{\prime}$-band photometry matches to within 0.5 mag.

The morphological parameters in our catalog may be used, for example, to address questions of "Nature vs. Nurture", including the well-studied morphology-density relation (e.g., Kauffmann et al. 2004). Are galaxy morphologies dictated mainly by their formation epoch, or are they shaped more by their environment (e.g., cluster vs. field)? We may also investigate the contributions of different galaxy types to star formation rates (e.g., Wolf et al. 2005, Paper II).

\section{Bayesian Photometric Redshifts (BPZ)}

We obtained photometric redshifts of the objects in our catalog using an updated version of the Bayesian photometric redshift software BPZ (Benítez 2000). In addition to re-calibrated SED (spectral energy distribution) templates introduced in Benítez et al. 2004, this new version also produces an enhanced summary of the redshift probability distribution $P(z)$ for each galaxy, reporting up to three peaks where warranted, along with their widths and

relative probabilities. And in this paper, we advocate the addition of two new templates to 

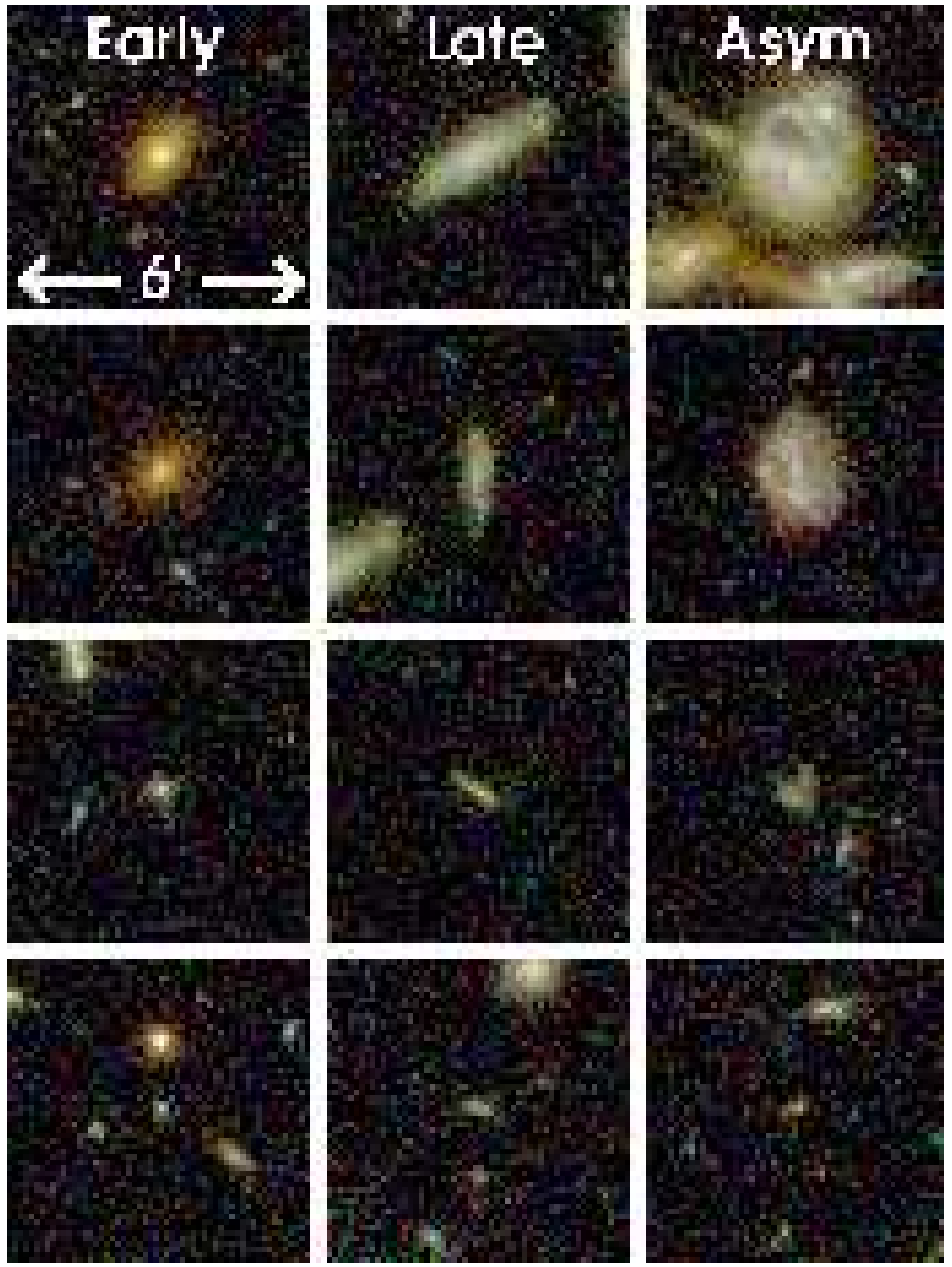

Fig. 10.- Examples of early type, late type, and highly asymmetrical galaxies. All postage stamps are $6^{\prime \prime} \times 6^{\prime \prime}$, taken from our $B V i^{\prime} z^{\prime}$ 4-color image. The first two columns show isolated and symmetrical galaxies with reliable measures of Sérsic index $\left(\sigma_{n} / n<1\right)$. Galaxies in the first column are morphologically classified as early $(n>2.5)$, while those in the second are classified as late $(n<2.5)$. The third column shows galaxies with clear asymmetries $(A>0.25)$. Galaxies in this column should not be classified by Sérsic index alone. Galaxy magnitudes range here range from roughly $i^{\prime} \sim 22.5$ to $i^{\prime} \sim 26.5$. 
the SED library $(\S 4.1)$.

We have experienced some numerical instabilities in BPZ for the extreme redshift and magnitude ranges present in the UDF. Future versions of BPZ will correct this problem, which lies in the normalization factor of the likelihood function $p(C \mid z, T) \propto F_{T T}(z)^{-1 / 2} \exp \left(-\frac{1}{2} \chi^{2}\left(z, T, a_{m}\right)\right)$ (Eq. 12 of Benítez 2000; $C$ represents the observed colors and $z, T, a_{m}$ are the model redshift, template, and amplitude, respectively). But for now, we simply remove the normalization factor, effectively reverting to the "frequentist" (ML) expression $p(C \mid z, T) \propto$ $\exp \left(-\frac{1}{2} \chi^{2}\left(z, T, a_{m}\right)\right)$. Of course every other aspect of the Bayesian method is retained, including the use of priors (which we have modified to accommodate our new templates).

Our BPZ catalog is available in Table 7 . Redshift probability distributions $P(z)$ are

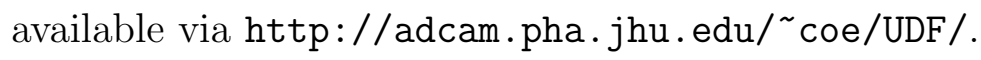


Table 7. Catalog: BPZ

\begin{tabular}{|c|c|c|c|c|c|c|c|c|c|c|c|c|c|c|}
\hline ID & $z_{b^{\mathrm{a}}}$ & $t_{b}{ }^{\mathrm{b}}$ & $\mathrm{ODDS}^{\mathrm{c}}$ & $\chi^{2 \mathrm{~d}}$ & $\chi_{\text {mod }}^{2}$ & $z_{b} 1^{\mathrm{f}}$ & $t_{b} 1^{\mathrm{b}}$ & $\mathrm{ODDS}^{\mathrm{g}}$ & $z_{b} 2^{\mathrm{f}}$ & $t_{b} 2^{\mathrm{b}}$ & ODDS2 ${ }^{\mathrm{g}}$ & $z_{b} 3^{\mathrm{f}}$ & $t_{b} 3^{\mathrm{b}}$ & ODDS3 $^{\mathrm{g}}$ \\
\hline 1 & $0.48 \pm 0.17$ & 3.67 & 1.000 & 2.429 & 0.087 & & & & & & & & & \\
\hline $2^{*}$ & $2.71_{-2.45}^{+0.49}$ & 6.00 & 0.500 & 0.118 & 0.669 & $2.71_{-0.55}^{+0.82}$ & 6.00 & 0.584 & $0.35_{-0.24}^{+0.47}$ & 6.67 & 0.095 & $\begin{array}{l}1.81_{-0.89}^{+0.35}\end{array}$ & 4.00 & 0.315 \\
\hline $3^{*}$ & $1.29_{-1.03}^{+1.31}$ & 7.33 & 0.359 & 0.147 & 0.689 & $\begin{array}{l}1.29_{-0.49}^{+1.78} \\
\end{array}$ & 7.33 & 0.873 & $0.55_{-0.54}^{+0.25}$ & 7.67 & 0.127 & $\ldots$ & $\ldots$ & $\ldots$ \\
\hline 4 & $3.80_{-0.87}^{+0.56}$ & 7.00 & 0.945 & 0.079 & 0.297 & $3.80_{-1.10}^{+0.52}$ & 7.00 & 0.984 & $0.32_{-0.16}^{+0.20}$ & 3.67 & 0.016 & & $\ldots$ & $\ldots$ \\
\hline 5 & $0.46_{-0.30}^{+2.78}$ & 6.00 & 0.592 & 0.586 & 0.431 & $0.46_{-0.08}^{+0.12}$ & 6.00 & 0.590 & $0.22_{-0.12}^{+0.08}$ & 5.00 & 0.180 & $3.18_{-0.21}^{+0.15}$ & 5.00 & 0.185 \\
\hline
\end{tabular}

Note. - Table 7 is published in its entirety in the electronic version of the Astronomical Journal. A portion is shown here for guidance regarding its form and content. The new version of BPZ summarizes each galaxy's redshift probability distribution $P(z)$ by giving the three highest peaks, where warranted. Here, galaxy $\# 1$ is well fit to a single redshift $z_{b}=0.48 \pm 0.17$ with ODDS $=1.0$ and $\chi_{\text {mod }}^{2}=0.087$. Galaxy \#2* instead may be anywhere between $2.71_{-2.45}^{+0.49}$ (95\% confidence limits). The three most likely redshifts for galaxy \# $2^{*}$ are given along with the redshift ranges for each peak and the fractions of $P(z)$ within those ranges. Due to space limitations, the last two columns of the table are not shown: $z_{M L} \& t_{M L}$ the maximum-likelihood redshift and SED fit.

${ }^{a}$ Most likely redshift and $95 \%$ confidence interval.

bED fit: $1=\mathrm{El}, 8=25 \mathrm{Myr}$ (Fig. 11)

${ }^{\mathrm{c}} P(z)$ contained within $0.12\left(1+z_{b}\right)$

${ }^{\mathrm{d}}$ Poorness of BPZ fit: observed vs. model fluxes.

e Modified $\chi^{2}$ : model fluxes given error bars.

${ }^{\mathrm{f}}$ Top three most likely redshifts and ranges.

${ }^{\mathrm{g}} P(z)$ contained within the redshift range of each peak. 


\subsection{Faint Blue Galaxy SEDs}

The SED template library of Benítez (2000) includes six templates for photometric redshifts, namely the Coleman et al. (1980) templates (used, for example, by FLY99 in their analysis of the HDF-N), plus two starburst templates from Kinney et al. (1996). These starburst templates were added to accommodate a population of "faint blue" galaxies revealed in the HDF-N. The addition of these templates significantly improved the accuracy of the photometric redshifts measured in the HDF-N (Benítez 2000).

The vast majority of galaxies in the HDF-N catalog (FLY99) can be roughly fit to one of these six templates (hereafter CWW+SB, Fig. 11). However, there are systematic differences between the observed and predicted colors of galaxies not only in the HDF-N catalog, but also in other spectroscopic catalogs. This issue was addressed in Benítez et al. (2004). The shapes of the CWW+SB templates were re-calibrated to more accurately reflect observed galaxy colors.

But with the increased depth of the UDF, we have discovered a large population of galaxies even "bluer" than those observed in the HDF-N (Fig. 12), and bluer than any of the (re-calibrated) CWW+SB templates (see Fig. 13). We are compelled to add SED templates to fit these galaxies.

This time we turn to GALAXEV, the synthetic template set produced and released by Bruzual \& Charlot (2003, hereafter BC03). The simple stellar population "SSP" models of BC03 span ages from $5 \mathrm{Myr}$ to $12 \mathrm{Gyr}$ and have metallicities of $Z=0.08,0.2$, and 0.5 (i.e., $Z=0.4 Z_{\odot}, Z_{\odot}$, and $\left.2.5 Z_{\odot}\right)$.

We experiment with the BC03 templates using the extensive spectroscopic redshift library of $1,800+$ galaxies in the GOODS-N field (Cowie et al. 2004; Wirth et al. 2004). High quality ACS $B V i^{\prime} z^{\prime}$ photometry for these galaxies is available via

http://www.stsci.edu/science/goods/ (Giavalisco et al. 2004). We first note that 3\% of these galaxies are in fact bluer than the CWW+SB templates. When we run BPZ on the GOODS-N photometry with the CWW+SB templates, our photometric redshifts match the spectroscopic redshifts with an RMS of $\Delta z=0.06\left(1+z_{\text {spec }}\right)$.

We then add BC03 templates to our CWW+SB template set one at a time to see if the accuracy and reliability improve. We also note how "popular" a given template is, i.e. how many galaxies "choose" the template as their best fit over the other six CWW+SB templates. The prior assigned to the template is exactly the same as that applied to our two SB templates. We set INTERP=2, so that two templates are interpolated between each set of adjacent templates. 


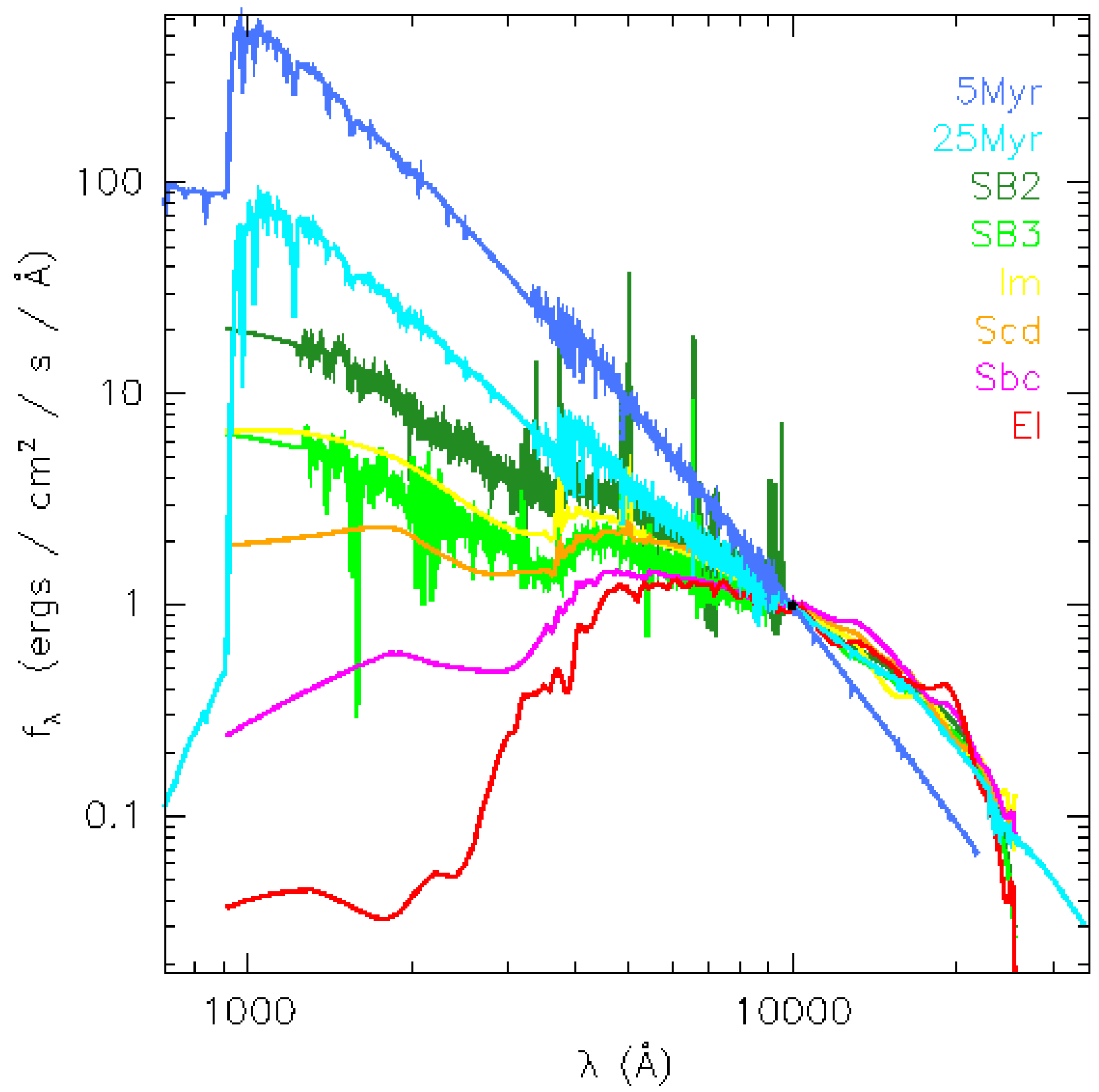

Fig. 11. - SED template set used with BPZ in this paper. All SEDs are normalized to $F_{\lambda}=1$ at $\lambda=10,000 \AA$. The bottom 6 are from Benítez et al. (2004). They are modified versions of the "CWW+SB" templates: El, Sbc, Scd, \& Im from Coleman et al. (1980) and SB3 \& SB2 starburst galaxies from Kinney et al. (1996). The steep ("blue") 25Myr \& 5Myr "SSP" SEDs (Bruzual \& Charlot 2003) have been added to accommodate the large population of faint blue galaxies observed in the UDF. Between each set of adjacent templates, we interpolate an additional two (not shown). 


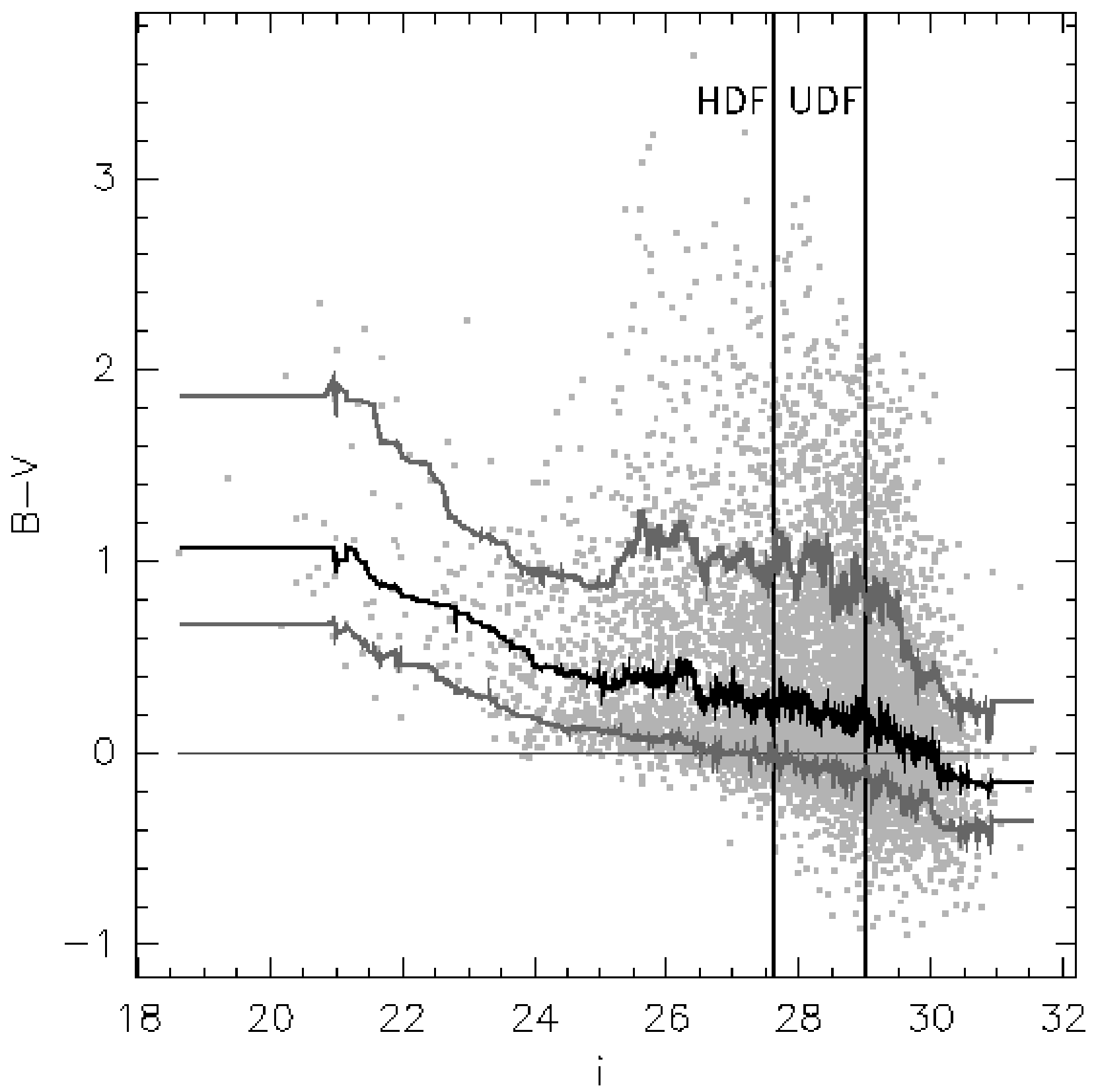

Fig. 12. $-B-V$ vs. $i^{\prime}$ for galaxies (stellarity $<0.8$ ) in our $10-\sigma$ catalog. The red line is a moving average (median) of 200 galaxies (or as few as 25 at the edges), while the magenta lines contain $68 \%(1-\sigma)$ of the galaxies. The vertical lines indicate the 10- $\sigma$ detection limits for the HDF and UDF in the $i^{\prime}$-band (0.5sq" aperture). As we probe to fainter magnitudes, we encounter bluer galaxies. 

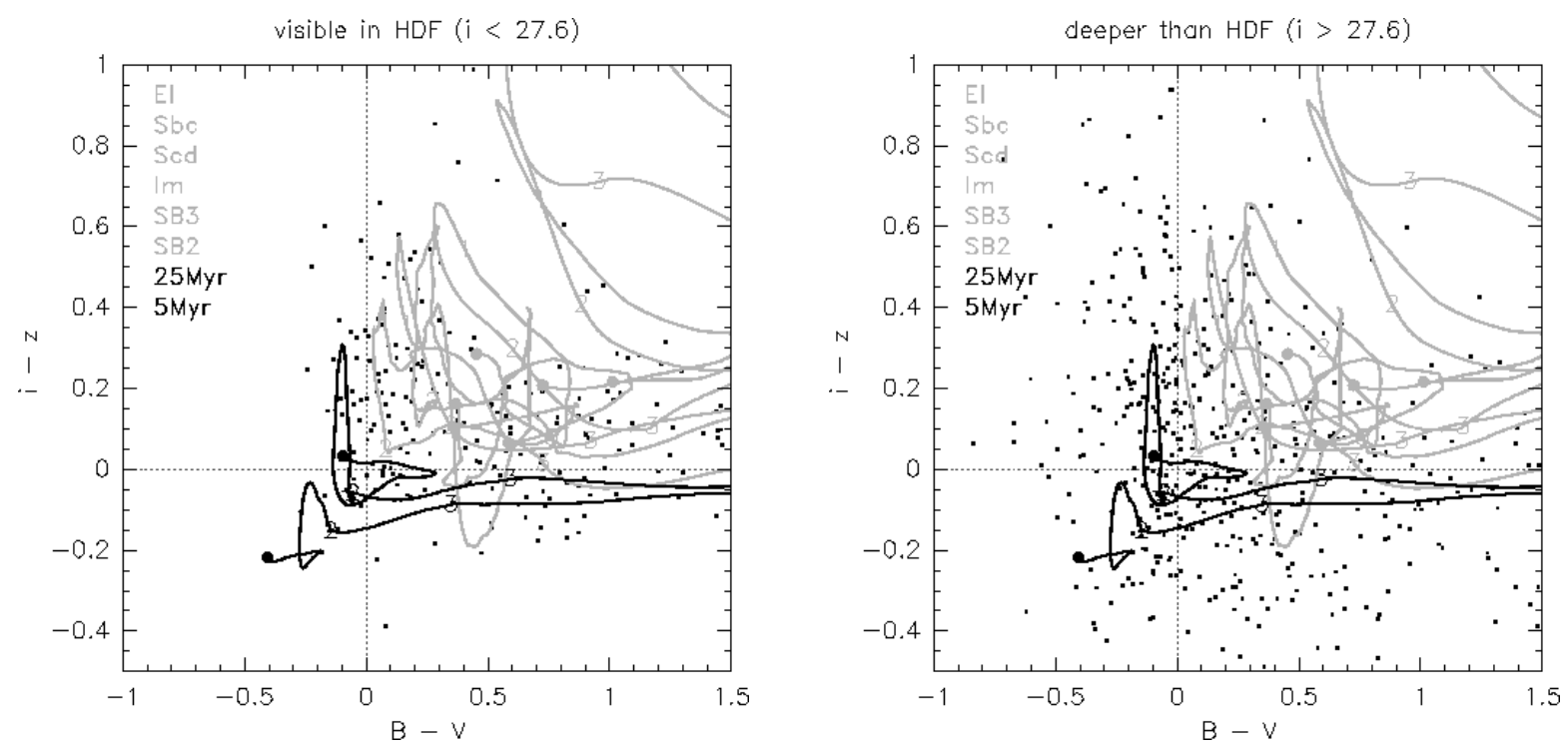

Fig. 13.- Color-color tracks for our SED templates plotted against observed colors of galaxies brighter (left) and fainter (right) than the HDF $i^{\prime}$-band detection limit $i^{\prime}=27.6$. For clarity, only 1/10 of the galaxies are plotted (small black squares). Each template's color-color track begins with a colored circle at $z=0$, and numbers along the track indicate other redshifts (most of these numbers are lost in the clutter of the plots). The young starburst BC03 templates (25Myr \& 5Myr) are required to fit the colors of the faint blue galaxy population revealed in the UDF. (These templates also slightly improve photo-z determinations in the HDF.) 
The "best" template is the $5 \mathrm{Myr}$ old $Z=0.08=0.4 Z_{\odot}$ SSP template. The addition of this template improves the accuracy of the photo-z's to an RMS of $\Delta z=0.04\left(1+z_{\text {spec }}\right)$. The "second best" template is the $25 \mathrm{Myr}$ old $Z=0.08 \mathrm{SSP}$ template. Both of these templates are bluer than the CWW+SB template set. Adding more templates does not improve the results, in fact it slightly worsens them (see Benítez 2000 for a discussion about the risks and meager benefits of including larger number of templates in the spectral library). Therefore, we decide we will incorporate both of these templates into our BPZ analysis of the UDF, with the reasonable expectation that they will describe a significant fraction of the very blue galaxy population. Our final set of 8 templates is shown in Fig. 11.

We expect our UDF BPZ accuracy to be $\Delta z=0.04\left(1+z_{\text {spec }}\right)$ or better, given the results obtained with the GOODS-N field, which do not include near-IR photometry. Our GOODS$\mathrm{N}$ BPZ results selected for ODDS $\geq 0.99$ has a catastrophic error rate of $<1 \%$, and that includes objects misclassified because they have AGN spectra. Of course it would be unwarranted to extend this statistic to magnitudes much fainter than the spectroscopic redshift limit, but it gives a good idea of the robustness of the BPZ results.

Unfortunately, a proper analysis of the nature of these faint blue galaxies is beyond the scope of this paper. Their redshift distribution is given in $\S 4.3$ and their contribution to the star formation rate density over time will be discussed in Paper II.

\subsection{Comparison with Spectroscopic Redshifts and COMBO-17}

\subsubsection{Spectroscopic Redshift Catalog}

Alessandro Rettura at ESO has compiled a list of all of the publicly available spectroscopic redshifts within the CDF-S. ${ }^{15} 76$ of these galaxies (and 3 stars) fall within the UDF ACS FOV. ${ }^{16}$ The GOODS VLT/FORS2 survey (Vanzella et al. 2005) obtained 22 of these redshifts. 20 of these are considered "solid" or "likely" (quality flags "A" or "B"). The VIMOS VLT Deep Survey (hereafter, VVDS; Le Fèvre et al. 2004) contributes another 41 redshifts, 25 of these being assigned $95 \%$ or $100 \%$ confidence. 7 redshifts come from Szokoly et al. (2004). 4 of these are deemed "reliable" (quality flags " 2 " or " $2+$ "). These 3 surveys yield $20+25+4=49$ "confident" redshifts. To those 49 , we add 6 redshifts which were not as-

\footnotetext{
${ }^{15}$ http://www.eso.org/science/goods/spectroscopy/ CDFS_Mastercat/
}

${ }^{16}$ Another 3 galaxies are either on or near the edge of the ACS FOV. These yield magnitudes in only 2 ACS filters, and we discard them in this paper. 
signed confidence levels: 5 obtained by Croom et al. (2001) and a $z=1.30$ type Ia supernova named "Aphrodite" by Strolger et al. (2004). Our final catalog contains 55 spectroscopic redshifts shown in Table 8 along with our photometry measurements and BPZ results. 
Table 8. Galaxies with confident spectroscopic redshifts in the UDF

\begin{tabular}{|c|c|c|c|c|c|c|c|c|c|c|c|c|}
\hline ID & \multicolumn{2}{|c|}{ RA \& DEC (J2000) } & Survey & $z_{\text {spec }}$ & $z_{b}$ & $\chi_{\text {mod }}^{2}$ & $B_{435}$ & $V_{606}$ & $i^{\prime} 775$ & $z^{\prime} 850$ & $J_{110}$ & $H_{160}$ \\
\hline $3088^{*}$ & $03: 32: 36.432$ & $-27: 47: 50.64$ & FORS2 & 0.127 & $0.19_{-0.14}^{+0.25}$ & 0.12 & $23.68 \pm 0.01$ & $22.85 \pm 0.00$ & $22.48 \pm 0.00$ & $22.37 \pm 0.00$ & $22.25 \pm 0.05$ & $22.08 \pm 0.06$ \\
\hline 57290 & $03: 32: 42.576$ & $-27: 45: 50.04$ & FORS2 & 0.218 & $0.30_{-0.21}^{+0.15}$ & 0.00 & $23.59 \pm 0.01$ & $\ldots$ & $22.36 \pm 0.00$ & $22.19 \pm 0.00$ & & $\cdots$ \\
\hline 1375 & 03:32:33.000 & $-27: 48: 29.52$ & FORS2 & 0.664 & $0.58 \pm 0.19$ & 0.16 & $24.27 \pm 0.01$ & $23.64 \pm 0.00$ & $23.01 \pm 0.00$ & $22.89 \pm 0.00$ & & $\ldots$ \\
\hline 8810 & $03: 32: 37.248$ & $-27: 46: 10.20$ & FORS2 & 0.736 & $0.72 \pm 0.20$ & 2.48 & $3.65 \pm 0.01$ & $23.10 \pm 0.00$ & $22.38 \pm 0.00$ & $22.11 \pm 0.00$ & $21.81 \pm 0.05$ & $21.45 \pm 0.06$ \\
\hline $4142^{*}$ & $03: 32: 44.208$ & $-27: 47: 33.36$ & FORS2 & 0.737 & $0.67 \pm 0.20$ & 0.16 & $22.93 \pm 0.00$ & $22.32 \pm 0.00$ & $21.66 \pm 0.00$ & $21.45 \pm 0.00$ & $21.21 \pm 0.05$ & $20.95 \pm 0.06$ \\
\hline 6206 & $03: 32: 38.496$ & $-27: 47: 02.40$ & FORS2 & 0.954 & $0.92 \pm 0.23$ & 0.02 & $24.70 \pm 0.02$ & $23.40 \pm 0.00$ & $21.94 \pm 0.00$ & $21.20 \pm 0.00$ & $20.71 \pm 0.05$ & $20.10 \pm 0.06$ \\
\hline $153^{*}$ & $03: 32: 39.600$ & $-27: 49: 09.48$ & FORS2 & 0.980 & $0.88 \pm 0.22$ & 0.03 & $25.06 \pm 0.04$ & $22.85 \pm 0.00$ & $21.43 \pm 0.00$ & $20.57 \pm 0.00$ & $\ldots$ & $\ldots$ \\
\hline 8261 & $03: 32: 35.784$ & $-27: 46: 27.48$ & FORS2 & 1.094 & $1.02 \pm 0.24$ & 0.04 & $25.75 \pm 0.03$ & $24.65 \pm 0.01$ & $23.53 \pm 0.00$ & $22.67 \pm 0.00$ & $\ldots$ & $\ldots$ \\
\hline $9264^{*}$ & $03: 32: 37.200$ & $-27: 46: 08.04$ & FORS2 & 1.096 & $1.17 \pm 0.26$ & 0.00 & $25.26 \pm 0.04$ & $23.41 \pm 0.01$ & $21.91 \pm 0.00$ & $20.84 \pm 0.00$ & $\ldots$ & $\ldots$ \\
\hline 8749 & $03: 32: 34.848$ & $-27: 46: 40.44$ & FORS2 & 1.099 & $0.82 \pm 0.21$ & 0.54 & $25.14 \pm 0.01$ & $24.21 \pm 0.00$ & $23.33 \pm 0.00$ & $22.71 \pm 0.00$ & $\ldots$ & $\ldots$ \\
\hline $4816^{*}$ & $03: 32: 44.184$ & $-27: 47: 29.40$ & FORS2 & 1.220 & $1.40 \pm 0.28$ & 0.52 & $24.93 \pm 0.02$ & $24.51 \pm 0.01$ & $23.97 \pm 0.00$ & $23.43 \pm 0.01$ & $22.88 \pm 0.06$ & $22.16 \pm 0.07$ \\
\hline $4396^{*}$ & $03: 32: 35.784$ & $-27: 47: 34.80$ & FORS2 & 1.223 & $1.26 \pm 0.27$ & 0.16 & $25.76 \pm 0.04$ & $25.34 \pm 0.02$ & $24.36 \pm 0.01$ & $23.57 \pm 0.01$ & $22.82 \pm$ & $22.11 \pm 0.07$ \\
\hline 1829 & $03: 32: 40.920$ & $-27: 48: 23.76$ & FORS2 & 1.244 & $1.29 \pm$ & 3.29 & $25.47 \pm 0.01$ & $25.34 \pm 0.01$ & $25.07 \pm 0.01$ & $24.45 \pm 0.01$ & $24.38 \pm 0.10$ & $24.22 \pm 0.12$ \\
\hline 1266 & $03: 32: 34.824$ & $-27: 48: 35.64$ & FORS2 & 1.245 & $1.40 \pm 0.28$ & 0.01 & $24.67 \pm 0.01$ & $24.25 \pm 0.00$ & $23.65 \pm 0.00$ & $22.97 \pm 0.00$ & $\ldots$ & $\ldots$ \\
\hline 7995 & $03: 32: 42.264$ & $-27: 46: 25.32$ & FORS2 & 1.288 & $1.26 \pm 0.27$ & 0.04 & $23.86 \pm 0.01$ & $23.58 \pm 0.00$ & $23.19 \pm 0.00$ & $22.53 \pm 0.00$ & $\ldots$ & $\ldots$ \\
\hline $6188^{*}$ & $03: 32: 42.384$ & $-27: 47: 07.80$ & FORS2 & 1.314 & $1.15 \pm 0.25$ & 3.62 & $26.57 \pm 0.09$ & $25.34 \pm 0.02$ & $24.14 \pm 0.01$ & $23.07 \pm 0.00$ & $22.18 \pm 0.05$ & $21.35 \pm 0.06$ \\
\hline 7725 & $03: 32: 35.088$ & $-27: 46: 15.60$ & FORS2 & 1.316 & $1.31 \pm 0.27$ & 0.00 & $24.35 \pm 0.01$ & $24.11 \pm 0.00$ & $23.73 \pm 0.00$ & $23.11 \pm 0.00$ & & $\ldots$ \\
\hline $6027^{*}$ & $03: 32: 39.648$ & $-27: 47: 09.24$ & FORS2 & 1.317 & $1.17 \pm 0.26$ & 0.31 & $26.01 \pm 0.06$ & $24.84 \pm 0.01$ & $23.63 \pm 0.00$ & $22.68 \pm 0.00$ & $21.80 \pm 0.05$ & $21.10 \pm 0.06$ \\
\hline 8461 & $03: 32: 44.616$ & $-27: 46: 32.16$ & FORS2 & 1.426 & $1.08_{-0.24}^{+0.47}$ & 0.04 & $24.39 \pm 0.01$ & $24.10 \pm 0.00$ & $23.70 \pm 0.00$ & $23.22 \pm 0.00$ & $\ldots$ & $\ldots$ \\
\hline $2225^{*}$ & 03:32:40.008 & $-27: 48: 15.12$ & FORS2 & 5.820 & $5.76 \pm 0.80$ & 0.13 & $>30.41$ & $29.34 \pm 0.25$ & $26.69 \pm 0.03$ & $25.11 \pm 0.01$ & $25.09 \pm 0.09$ & $25.19 \pm 0.10$ \\
\hline 5670 & $03: 32: 46.536$ & $-27: 47: 08.88$ & VVDS & 0.128 & $0.23_{-0.14}^{+0.17}$ & 0.00 & $22.11 \pm 0.00$ & $21.23 \pm 0.00$ & $20.84 \pm 0.00$ & $20.69 \pm 0.00$ & & $\cdots$ \\
\hline $1971^{*}$ & $03: 32: 41.928$ & $-27: 47: 57.48$ & VVDS & 0.151 & $0.17 \pm 0.14$ & 0.22 & $21.12 \pm 0.00$ & $20.46 \pm 0.00$ & $20.18 \pm 0.00$ & $20.09 \pm 0.00$ & $19.98 \pm 0.05$ & $19.80 \pm 0.06$ \\
\hline 5620 & $03: 32: 43.560$ & $-27: 47: 16.80$ & VVDS & 0.212 & $0.22 \pm 0.14$ & 0.86 & $23.87 \pm 0.00$ & $23.42 \pm 0.00$ & $23.34 \pm 0.00$ & $23.41 \pm 0.00$ & $23.28 \pm 0.07$ & $23.34 \pm 0.08$ \\
\hline 1000 & $03: 32: 36.744$ & $-27: 48: 43.56$ & VVDS & 0.213 & $3.13_{-2.95}^{+0.49}$ & 1.23 & $23.86 \pm 0.00$ & $23.39 \pm 0.00$ & $23.30 \pm 0.00$ & $23.40 \pm 0.00$ & & .. \\
\hline 5606 & $03: 32: 34.104$ & $-27: 47: 12.12$ & VVDS & 0.226 & $0.17 \pm 0.14$ & 0.06 & $22.11 \pm 0.00$ & $21.14 \pm 0.00$ & $20.73 \pm 0.00$ & $20.59 \pm 0.00$ & $20.36 \pm 0.05$ & $20.10 \pm 0.06$ \\
\hline 5190 & $03: 32: 34.824$ & $-27: 47: 21.84$ & VVDS & 0.315 & $1.23 \pm 0.26$ & 0.30 & $24.16 \pm 0.01$ & $23.97 \pm 0.00$ & $23.67 \pm 0.00$ & $23.13 \pm 0.00$ & $22.88 \pm 0.07$ & $22.56 \pm 0.08$ \\
\hline 7847 & $03: 32: 41.760$ & $-27: 46: 19.56$ & VVDS & 0.334 & $0.38 \pm 0.16$ & 0.02 & $23.60 \pm 0.01$ & $22.00 \pm 0.00$ & $21.25 \pm 0.00$ & $20.90 \pm 0.00$ & & $\ldots$ \\
\hline 3492 & $03: 32: 45.072$ & $-27: 47: 38.40$ & VVDS & 0.345 & $0.29 \pm 0.15$ & 0.67 & $21.75 \pm 0.00$ & $20.83 \pm 0.00$ & $20.58 \pm 0.00$ & $20.39 \pm 0.00$ & $20.32 \pm 0.05$ & $20.13 \pm 0.06$ \\
\hline $4267^{*}$ & 03:32:48.336 & $-27: 47: 38.76$ & VVDS & 0.347 & $3.15 \pm 0.49$ & 0.06 & $25.47 \pm 0.02$ & $24.63 \pm 0.01$ & $24.45 \pm 0.01$ & $24.42 \pm 0.01$ & $\ldots$ & $\ldots$ \\
\hline 3268 & $03: 32: 41.400$ & $-27: 47: 47.04$ & VVDS & 0.347 & $0.30 \pm 0.15$ & 0.36 & $22.96 \pm 0.00$ & $22.11 \pm 0.00$ & $21.84 \pm 0.00$ & $21.66 \pm 0.00$ & $21.56 \pm 0.05$ & $21.37 \pm 0.06$ \\
\hline $8585^{*}$ & $03: 32: 35.496$ & $-27: 46: 27.12$ & VVDS & 0.377 & $1.00 \pm 0.24$ & 0.00 & $22.36 \pm 0.00$ & $22.07 \pm 0.00$ & $21.58 \pm 0.00$ & $21.11 \pm 0.00$ & $\cdots$ & $\ldots$ \\
\hline $900^{*}$ & $03: 32: 44.448$ & $-27: 48: 19.08$ & VVDS & 0.417 & $0.43 \pm 0.17$ & 0.06 & $22.29 \pm 0.00$ & $21.07 \pm 0.00$ & $20.39 \pm 0.00$ & $20.06 \pm 0.00$ & $\because$ & \\
\hline 4929 & $03: 32: 45.120$ & $-27: 47: 24.00$ & VVDS & 0.4 & $0.50 \pm$ & 1.43 & $22.73 \pm 0.00$ & $21.53 \pm 0.00$ & $20.82 \pm 0.00$ & $20.43 \pm 0.00$ & $20.09 \pm 0.05$ & $19.57 \pm 0.06$ \\
\hline 2107 & $03: 32: 45.792$ & $-27: 48: 12.96$ & VVDS & 0.534 & $0.56 \pm$ & 0.02 & $24.38 \pm 0.01$ & $22.76 \pm 0.00$ & $21.75 \pm 0.00$ & $21.36 \pm 0.00$ & $\cdots$ & . \\
\hline $6747^{*}$ & $03: 32: 38.784$ & $-27: 46: 48.72$ & VVDS & 0.619 & $0.56 \pm 0.18$ & 0.82 & $25.01 \pm 0.03$ & $22.95 \pm 0.00$ & $21.67 \pm 0.00$ & $21.22 \pm 0.00$ & $20.75 \pm 0.05$ & $20.21 \pm 0.06$ \\
\hline
\end{tabular}


Table 8-Continued

\begin{tabular}{|c|c|c|c|c|c|c|c|c|c|c|c|c|}
\hline ID & $\mathrm{RA} \& \mathrm{DE}$ & $(\mathrm{J} 2000)$ & Survey & $z_{\text {spec }}$ & $z_{b}$ & $\chi_{\text {mod }}^{2}$ & $B_{435}$ & $V_{606}$ & $i^{\prime}{ }_{775}$ & $z_{850}^{\prime}$ & $J_{110}$ & $H_{160}$ \\
\hline 2607 & $03: 32: 43.248$ & $-27: 47: 56.04$ & VVDS & 0.666 & $0.63 \pm 0.19$ & 1.10 & $23.06 \pm 0.01$ & $21.94 \pm 0.00$ & $20.99 \pm 0.00$ & $20.66 \pm 0.00$ & $20.28 \pm 0.05$ & $19.77 \pm 0.06$ \\
\hline 968 & $03: 32: 37.536$ & $-27: 48: 38.88$ & VVDS & 0.666 & $0.58 \pm 0.19$ & 0.12 & $22.17 \pm 0.00$ & $21.56 \pm 0.00$ & $20.96 \pm 0.00$ & $20.84 \pm 0.00$ & $20.63 \pm 0.05$ & $20.38 \pm 0.06$ \\
\hline $662^{*}$ & 03:32:41.880 & $-27: 48: 54.00$ & VVDS & 0.666 & $0.58 \pm 0.19$ & 0.01 & $23.17 \pm 0.00$ & $22.56 \pm 0.00$ & $21.96 \pm 0.00$ & $21.85 \pm 0.00$ & $\ldots$ & $\ldots$ \\
\hline 355 & 03:32:38.808 & $-27: 49: 09.48$ & VVDS & 0.666 & $0.60 \pm 0.19$ & 0.17 & $24.49 \pm 0.01$ & $23.69 \pm 0.00$ & $22.92 \pm 0.00$ & $22.72 \pm 0.00$ & $\ldots$ & $\ldots$ \\
\hline 53380 & $03: 32: 29.952$ & $-27: 47: 57.12$ & VVDS & 0.667 & $0.62 \pm 0.19$ & 0.07 & $25.50 \pm 0.04$ & $23.87 \pm 0.01$ & $22.68 \pm 0.00$ & $22.19 \pm 0.00$ & $\ldots$ & $\ldots$ \\
\hline 6933 & 03:32:33.432 & $-27: 46: 50.52$ & VVDS & 0.733 & $0.61 \pm 0.19$ & 0.01 & $24.18 \pm 0.01$ & $23.67 \pm 0.00$ & $23.05 \pm 0.00$ & $22.90 \pm 0.00$ & $\ldots$ & $\ldots$ \\
\hline 2525 & $03: 32: 43.584$ & $-27: 48: 04.68$ & VVDS & 0.736 & $0.67 \pm 0.20$ & 0.02 & $24.21 \pm 0.01$ & $23.52 \pm 0.00$ & $22.72 \pm 0.00$ & $22.50 \pm 0.00$ & $22.31 \pm 0.06$ & $22.02 \pm 0.07$ \\
\hline $3372^{*}$ & $03: 32: 42.288$ & $-27: 47: 45.96$ & VVDS & 0.996 & $0.81 \pm 0.21$ & 0.60 & $22.86 \pm 0.00$ & $22.32 \pm 0.00$ & $21.62 \pm 0.00$ & $21.23 \pm 0.00$ & $20.91 \pm 0.05$ & $20.58 \pm 0.06$ \\
\hline 5417 & 03:32:39.888 & $-27: 47: 15.00$ & VVDS & 1.095 & $0.99 \pm 0.23$ & 0.29 & $23.03 \pm 0.00$ & $22.57 \pm 0.00$ & $21.95 \pm 0.00$ & $21.44 \pm 0.00$ & $21.09 \pm 0.05$ & $20.73 \pm 0.06$ \\
\hline $797^{*}$ & $03: 32: 35.976$ & $-27: 48: 50.40$ & VVDS & 1.306 & $1.44 \pm 0.29$ & 0.00 & $22.37 \pm 0.00$ & $22.18 \pm 0.00$ & $21.99 \pm 0.00$ & $21.57 \pm 0.00$ & $\ldots$ & \\
\hline 4445 & $03: 32: 38.784$ & $-27: 47: 32.28$ & Szokoly & 0.456 & $0.07_{-0.07}^{+0.13}$ & 4.82 & $21.95 \pm 0.00$ & $21.50 \pm 0.00$ & $21.15 \pm 0.00$ & $20.91 \pm 0.00$ & $20.68 \pm 0.05$ & $20.33 \pm 0.06$ \\
\hline 4394 & $03: 32: 31.368$ & $-27: 47: 25.08$ & Szokoly & 0.665 & $0.60 \pm 0.19$ & 0.05 & $22.45 \pm 0.00$ & $21.78 \pm 0.00$ & $21.15 \pm 0.00$ & $21.00 \pm 0.00$ & $\ldots$ & $\ldots$ \\
\hline 8275 & $03: 32: 36.504$ & $-27: 46: 29.28$ & Szokoly & 0.764 & $0.70 \pm 0.20$ & 0.05 & $22.65 \pm 0.00$ & $22.13 \pm 0.00$ & $21.40 \pm 0.00$ & $21.20 \pm 0.00$ & $20.98 \pm 0.05$ & $20.75 \pm 0.06$ \\
\hline $865^{*}$ & $03: 32: 39.672$ & $-27: 48: 50.76$ & Szokoly & 3.064 & $3.67_{-3.34}^{+0.55}$ & 30.80 & $27.16 \pm 0.10$ & $25.30 \pm 0.01$ & $24.57 \pm 0.01$ & $24.39 \pm 0.01$ & $23.78 \pm 0.07$ & $22.32 \pm 0.08$ \\
\hline 8015 & $03: 32: 33.528$ & $-27: 46: 23.52$ & Croom & 0.276 & $0.34_{-0.23}^{+0.16}$ & 0.00 & $22.89 \pm 0.00$ & $21.78 \pm 0.00$ & $21.28 \pm 0.00$ & $21.05 \pm 0.00$ & $\ldots$ & \\
\hline 3822 & $03: 32: 44.856$ & $-27: 47: 27.60$ & Croom & 0.437 & $0.14 \pm 0.13$ & 0.02 & $20.18 \pm 0.00$ & $19.14 \pm 0.00$ & $18.62 \pm 0.00$ & $18.44 \pm 0.00$ & $18.09 \pm 0.05$ & $17.71 \pm 0.06$ \\
\hline $2387^{*}$ & $03: 32: 35.760$ & $-27: 47: 58.92$ & Croom & 0.665 & $0.63 \pm 0.19$ & 0.11 & $24.49 \pm 0.02$ & $22.14 \pm 0.00$ & $20.76 \pm 0.00$ & $20.31 \pm 0.00$ & $19.84 \pm 0.05$ & $19.27 \pm 0.06$ \\
\hline $4587^{*}$ & $03: 32: 40.656$ & $-27: 47: 30.84$ & Croom & 0.667 & $0.68 \pm 0.20$ & 0.26 & $24.79 \pm 0.02$ & $22.98 \pm 0.00$ & $21.69 \pm 0.00$ & $21.24 \pm 0.00$ & $20.76 \pm 0.05$ & $20.20 \pm 0.06$ \\
\hline $3677^{*}$ & 03:32:37.296 & $-27: 47: 29.40$ & Croom & 0.669 & $0.57 \pm 0.19$ & 2.45 & $23.59 \pm 0.01$ & $21.62 \pm 0.00$ & $20.23 \pm 0.00$ & $19.76 \pm 0.00$ & $19.25 \pm 0.05$ & $18.64 \pm 0.06$ \\
\hline 7705 & $03: 32: 37.560$ & $-27: 46: 46.56$ & Strolger & 1.300 & $1.33 \pm 0.27$ & 0.29 & $25.83 \pm 0.02$ & $25.82 \pm 0.01$ & $25.70 \pm 0.01$ & $25.15 \pm 0.01$ & $24.93 \pm 0.09$ & $24.77 \pm 0.10$ \\
\hline
\end{tabular}

Note. - Redshift surveys are FORS2 (Vanzella et al. 2005), VVDS (Le Fèvre et al. 2004), Szokoly et al. (2004), Croom et al. (2001), and Strolger et al. (2004). ID numbers below 41000 correspond to B04 \& T04 detections; asterisks $\left.*^{*}\right)$ indicate that object definitions have been altered $(\S 3.1)$. $z_{b}$ gives the peak of the Bayesian photometric redshift distribution $P(z)$ along with a $95 \%$ confidence interval, while $\chi_{m o d}^{2}$ measures how poorly the best fitting SED template at $z_{b}$ fits the observed colors. Magnitudes are "total" AB magnitudes with isophotal colors: NIC3 magnitudes are corrected to the PSF of the ACS images (§3.3). We have also applied offsets of $(J:-0.30 \pm 0.03, H:-0.18 \pm 0.04)$ to the NIC3 magnitudes $(\S 4.2 .2)$. And all of our magnitudes have been corrected for galactic extinction (Table 2). Non-detections (listed, for example, as > 31.05) quote the 1- $\sigma$ detection limit of the aperture used on the given object. Magnitudes are left blank where objects are unobserved (outside the NIC3 FOV) or contain saturated or other bad pixels. Color images of these objects along with SED fits and more are available at http://adcam.pha.jhu.edu/ ${ }^{\sim}$ coe/UDF/zsconf/. 
The UDF ACS \& NIC3 FOVs were oriented to contain Strolger et al.'s supernova and a spectroscopically confirmed $z=5.8$ object, corresponding to our \#2225*. ${ }^{17}$ \#2225* was originally detected as an $i^{\prime}$-dropout by Stanway et al. (2003), and has since been known as SBM03\#1. Since then, multiple spectra have been taken of this object (Dickinson et al. 2004, Stanway et al. 2004, and most recently FORS2). The first two papers list SBM03\#1 at $z=5.83$, while FORS2 favors a slightly lower $z=5.82$. (We find $z_{b}=5.78 \pm 0.80$.)

Multiple spectra have been obtained for several other objects in the UDF as well. For all but two of these objects, the different authors claim nearly identical spectroscopic redshifts $(\Delta z<0.005)$. In both of the discrepant cases, the FORS2 authors reject the earlier " $75 \%$ confident" VVDS redshifts in favor of their own "solid" or "likely" redshifts, citing superior classification of emission lines. Our BPZ values also support the FORS2 values. These objects are our \#57290 $\left(z_{b}=0.31_{-.22}^{+.15}\right.$; FORS2 GDS J033242.56-274550.2 $z=0.218$; VVDS \#28150 $z=0.6354)$ and our \#6188* $\left(z_{b}=1.17 \pm 0.25\right.$; GDS J033242.38-274707.6 $z=1.314$; VVDS \#72036 z $=0.6885)$.

\subsubsection{UDF NIC3 Recalibration: Empirical Derivation}

Here we consider the 23 galaxies within the NIC3 FOV and with confident spectroscopic redshifts from FORS2 and VVDS. When BPZ SED templates are fit to the photometry of these galaxies, we find that the NIC3 fluxes are below those expected given the ACS fluxes and the known redshifts (see Fig. 14). We find weighted average magnitude offsets of $-0.30 \pm 0.03$ for $J$ and $-0.18 \pm 0.04$ for $H$. This appears to be a normal sample of relatively bright galaxies $\left(20.3<z^{\prime}<25.2\right.$, with half having $\left.z^{\prime}<21.7\right)$. Thus these magnitude biases cannot be explained by our choice of apertures or our "PSF corrections", as neither of these significantly affects the magnitudes of such bright objects (Fig. 6). The galaxies belong to all of our different SED types, which means that the problem cannot be traced to a single bad template. Few of the galaxies were fit to the new BC03 templates; most were instead classified as one of the widely used and well-calibrated CWW+SB templates (see $§ 4.1$ ).

To further test the UDF NIC3 calibration, we compared our photometry to photometry we obtained from the VLT $J$ image (Vandame et al., in prep.) ${ }^{18}$. As the PSF corrections are small for these bright objects, "quick and dirty" photometry is sufficient here. We simply

\footnotetext{
${ }^{17}$ Again, the asterisk $(*)$ indicates that B04's $i^{\prime}$-band segment for object \#2225 was altered. Here it was replaced with their $z^{\prime}$-band segment (\#31526).

${ }^{18}$ VLT observations have been carried out at the ESO Paranal Observatory under Program ID: LP168.A0485
} 
took our VLT MAG_AUTO measurements and added these to our main catalog, matching objects by position. (Further tests confirm that these "quick" magnitudes are accurate to within \pm 0.06 mags.) A straight comparison between the VLT $J$ and NIC3 $J$-band magnitudes shows that the NIC3 $J$-band magnitudes are about 0.3 magnitudes too faint. When we apply corrections to account for the different filter shapes (VLT $J$ vs. NIC3 $J$ ), this difference is slightly reduced, by 0.05 mags. (Note that these corrections require assumptions of redshift and SED for each galaxy.) Given the uncertainties involved, these results are consistent with our above analysis. We note that nearly identical offsets were independently derived for the UDF in a similar analysis by Gwyn \& Hartwick (2005, and priv. comm.). Similar deficits in NICMOS fluxes have been observed by Mobasher \& Riess (2005) in the UDF and by Adam Riess in observations of supernovae (priv. comm.).

Other UDF studies have not questioned the NIC3 calibration; this can be understood since the uncorrected magnitudes will often produce photometric redshifts which are roughly correct. For example, when we revert the NIC3 photometry of galaxy \#2525 to prerecalibration magnitudes, the derived redshift remains the same: $z_{b}=0.68$ (close to the spectroscopic value of $z_{\text {spec }}=0.74$ ). As we see in Fig. 15, the best fit SED simply "splits the difference" between NIC3 fluxes that are a bit too low and ACS fluxes that are a bit too high. (Compare to Fig. 14a, although keep in mind that the BPZ fit in that figure was constrained to the spectroscopic redshift $z_{b}=z_{\text {spec }}=0.74$.) Galaxies such as \#2525 with spectroscopic redshift available are sufficiently bright that accurate photometric redshifts may often be obtained with less than perfect photometry. But by looking for and correcting for magnitude offsets in the individual filters (Fig. 14b), we help ensure that our photometry is robust for the more challenging fainter galaxies.

Our offsets appear to be supported by a recent recalibration of the UDF NIC3 images combined with non-linearity measured in NICMOS itself (§3.4). Thus we are encouraged to proceed with our analysis given our derived offsets: $-0.30 \pm 0.03$ in $J$ and $-0.18 \pm 0.04$ in $H$.

\subsubsection{Spectroscopic Redshift Comparison}

After our recalibration of the UDF NIC3 photometry, our photometric redshifts agree very well with the 55 spectroscopic redshifts described in $§ 4.2 .1$ (see Fig. 16). Among the 41 galaxies with ODDS $\geq 0.95 \& \chi_{\text {mod }}^{2}<1$, we find an RMS of $\Delta z=0.04\left(1+z_{\text {spec }}\right)$, but only after we exclude 4 outliers. 3 of these outliers are from the VVDS, while one is from Croom et al. (2001) (and was not assigned a confidence level). The outliers are clearly visible in Fig. 16a. 

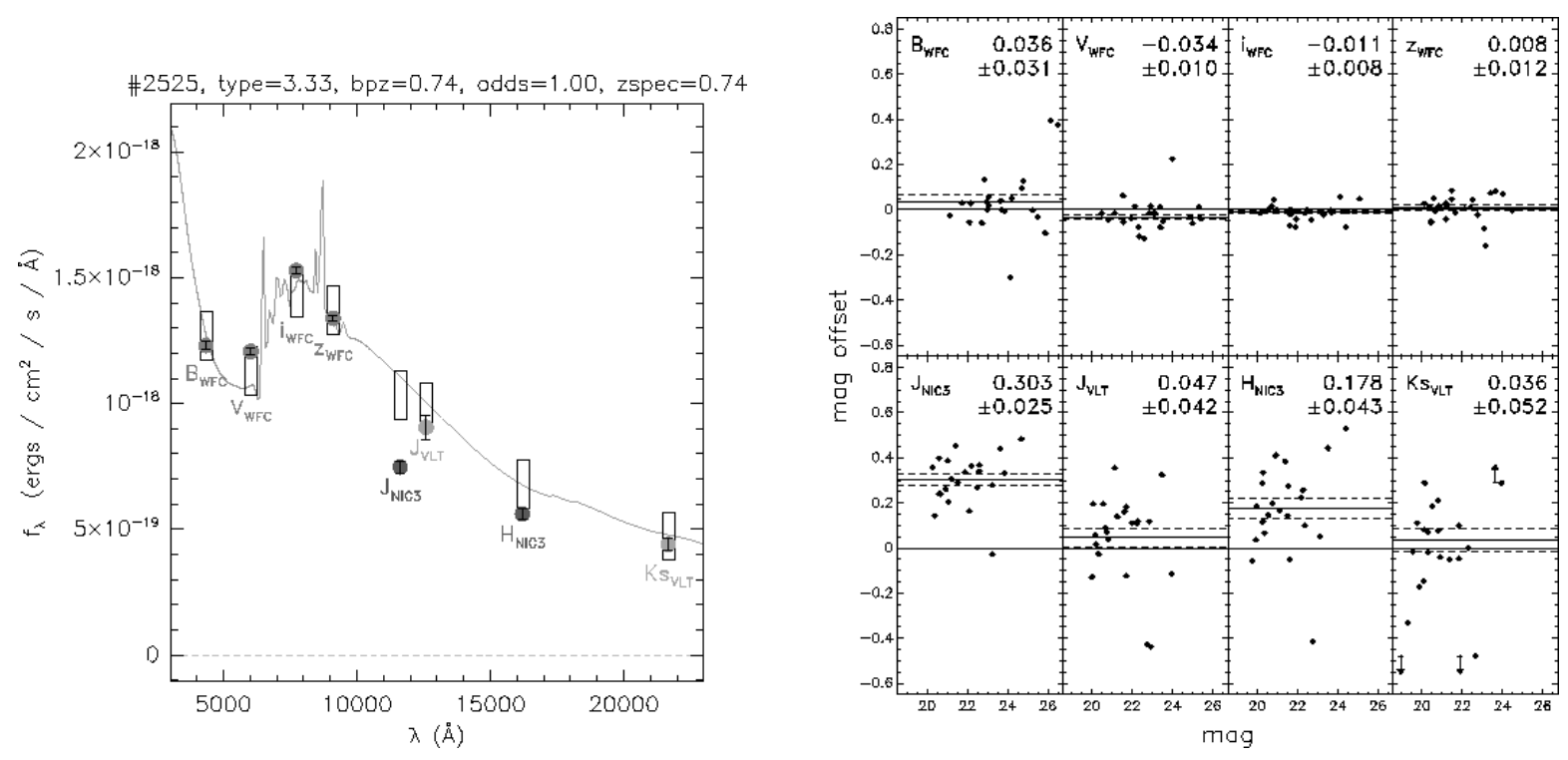

Fig. 14. - Left: BPZ SED fit to an object with a "95\% confident" spectroscopic redshift in the VVDS. We supplied BPZ with the known redshift, and BPZ chose to fit a hybrid Scd-Im SED to our observed fluxes (filled circles with filters labeled). The SED itself is drawn in black. It is integrated over each filter to yield the model fluxes shown as blue rectangles (given height to represent uncertainty, §4.3). The $J \& H$ NIC3 fluxes are too low to fit the SED (especially the $J$ flux). Meanwhile, the $J \& K_{s}$ VLT fluxes fit the SED well. This is typical of all of the galaxies with "confident" spectroscopic redshifts available, as we see in the plot on the right, where magnitude offsets are plotted versus magnitude for each filter. The solid red line indicates the $\sigma$-clipped mean of the offsets, while the dashed red lines demark the $\sigma$-clipped $1-\sigma$ scatter divided by $\sqrt{N}$. The ACS WFC and VLT magnitudes fit the SEDs well and have no significant offsets. But the NIC3 magnitudes have weighted average offsets of $0.30 \pm 0.03$ for $J$ and $0.18 \pm 0.04$ for $H$. We correct for these offsets by adjusting the NIC3 magnitudes. 


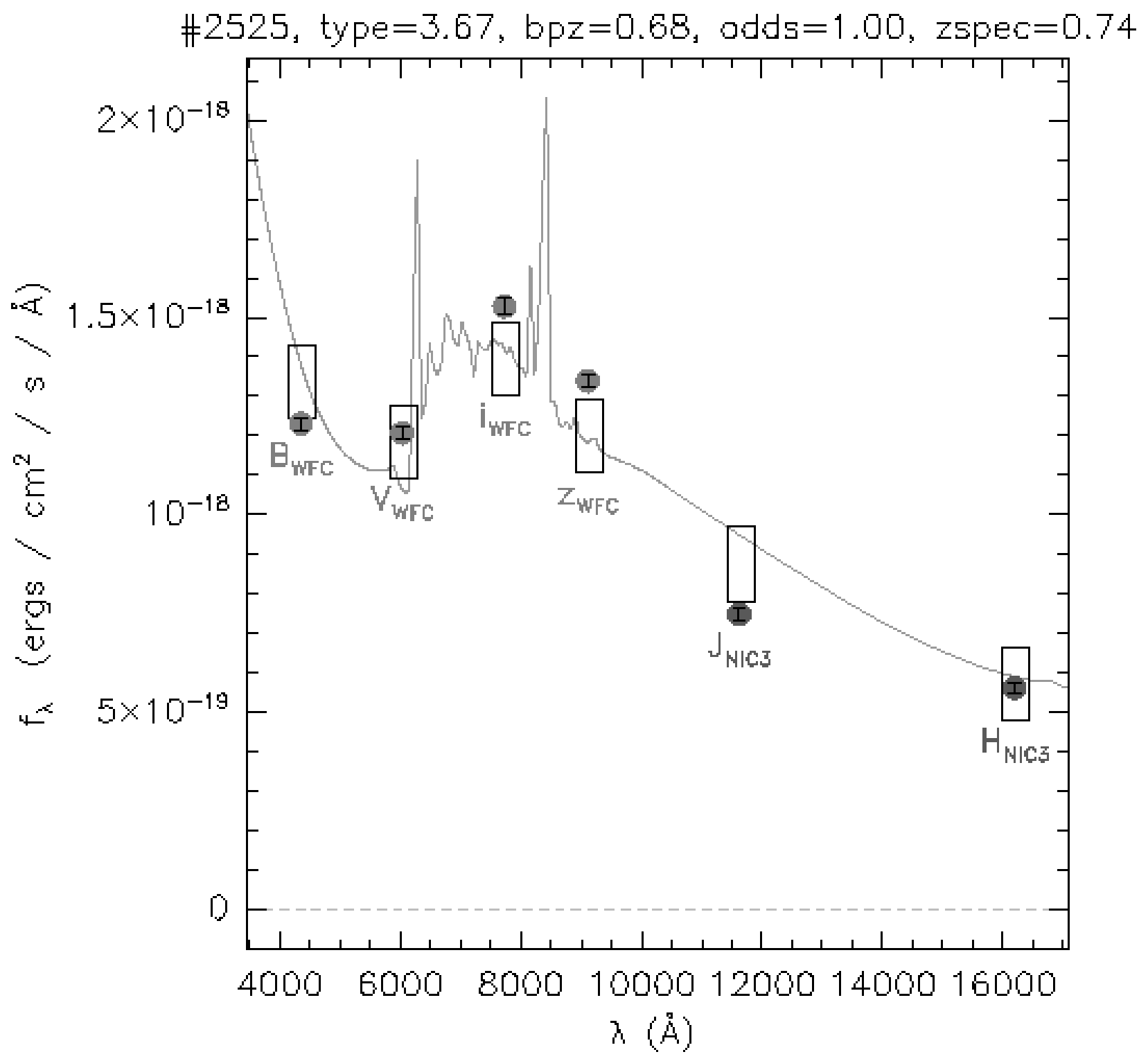

Fig. 15. - BPZ SED fit to \#2525 without our NIC3 magnitude offsets applied. The photometric redshift $z_{b}=0.68$ obtained for this galaxy is the same whether our offsets are applied or not. Here the best fitting SED simply "splits the difference" between ACS fluxes that are "too high" and NIC3 fluxes that are "too low". Compare to Fig. 14a (but keep in mind that the BPZ fit in that figure was constrained to the correct redshift $\left.z_{b}=z_{s p e c}=0.74\right)$ Accurate photometric redshifts are relatively easy to come by for bright galaxies such as this with a spectroscopic redshift available. Thus this agreement is no guarantee that the underlying photometry is robust. 
The $V i^{\prime} z^{\prime}$ filter set covers the entire spectral range covered by these spectroscopic surveys (for example, FORS2: 6000 - 10800A; VVDS: $5500-9500 \AA$ ). Inside the NIC3 FOV, our Bayesian photometric redshifts benefit from three "extra" filters, as the $B V i^{\prime} z^{\prime} J H$ filter set covers $\sim 4000-18000 \AA$. And of course the UDF photometry extends much deeper than the spectroscopic surveys. Thus, even when discrepancies do occur, it is unclear whether to favor the photometric or spectroscopic redshifts (especially for those spectroscopic redshifts that are assigned low confidence). In the case of the HDF, Fernández-Soto et al. (2001) showed that most of the discrepancies were likely the result of incorrect spectroscopic redshifts which were overruled by more reliable photometric redshifts.

Outside the NIC3 FOV, we expect the four ACS filters $\left(B V i^{\prime} z^{\prime}\right)$ to continue to deliver high quality photometric redshifts. Fig. 17b presents BPZ results obtained using only the ACS photometry for galaxies with spectroscopic redshifts and within the NIC3 FOV. The results are on par with those obtained using all 6 filters (Fig. 17a). (Of course galaxies with confident spectroscopic redshifts are relatively easy tests, as they are usually bright with dominant spectral features.)

\subsection{4. $C O M B O-17$}

We also found good agreement with most of the photometric redshifts obtained by the COMBO-17 survey (Wolf et al. 2004) for bright galaxies (Fig. 18). COMBO-17 covers a wide spectral range with very good resolution for a photometric redshift survey. But of course, it does not penetrate nearly as deep as the UDF; COMBO-17 only claims to yield reliable redshifts up to $R \lesssim 24$. In addition, COMBO-17 does not attempt to model galaxies beyond $z>1$.4. So any galaxies at $z>1.4$ will have been reassigned $z_{C O M B O-17}<1.4$. Of course as Wolf et al. (2004) point out, such bright galaxies are unlikely to be at $z>1.4$.

We find that our best fit redshifts $z_{b}$ agree well with those of COMBO-17 for $R \lesssim 23.7$ galaxies. The relationship is especially tight for $R<23$, with a few notable exceptions. Two $R \sim 22, z_{\text {СОм ВО-17 }} \sim 0.2$ galaxies $\left(\# 5491 \& \# 6082\right.$ ) are assigned $z_{b} \gtrsim 3$. (This redshift degeneracy is also documented for similar galaxies in Fig. 19.) These two galaxies truly stand out in our catalog. To be at $z \sim 3$ these $R \sim 22$ galaxies would have to be monsters $(M(1400 \AA) \sim-23$; see Paper II). (Note that BPZ's priors usually help to resolve such redshift degeneracies in favor of the more reasonable choice, given the galaxy's magnitude. But in the case of these two galaxies, the $z \sim 3$ fits were deemed sufficiently superior to rule out the more reasonable $z \sim 0.2$ fits.) Thus for these bright galaxies we are inclined to believe the COMBO-17 results, which benefit from observations in many more filters. 

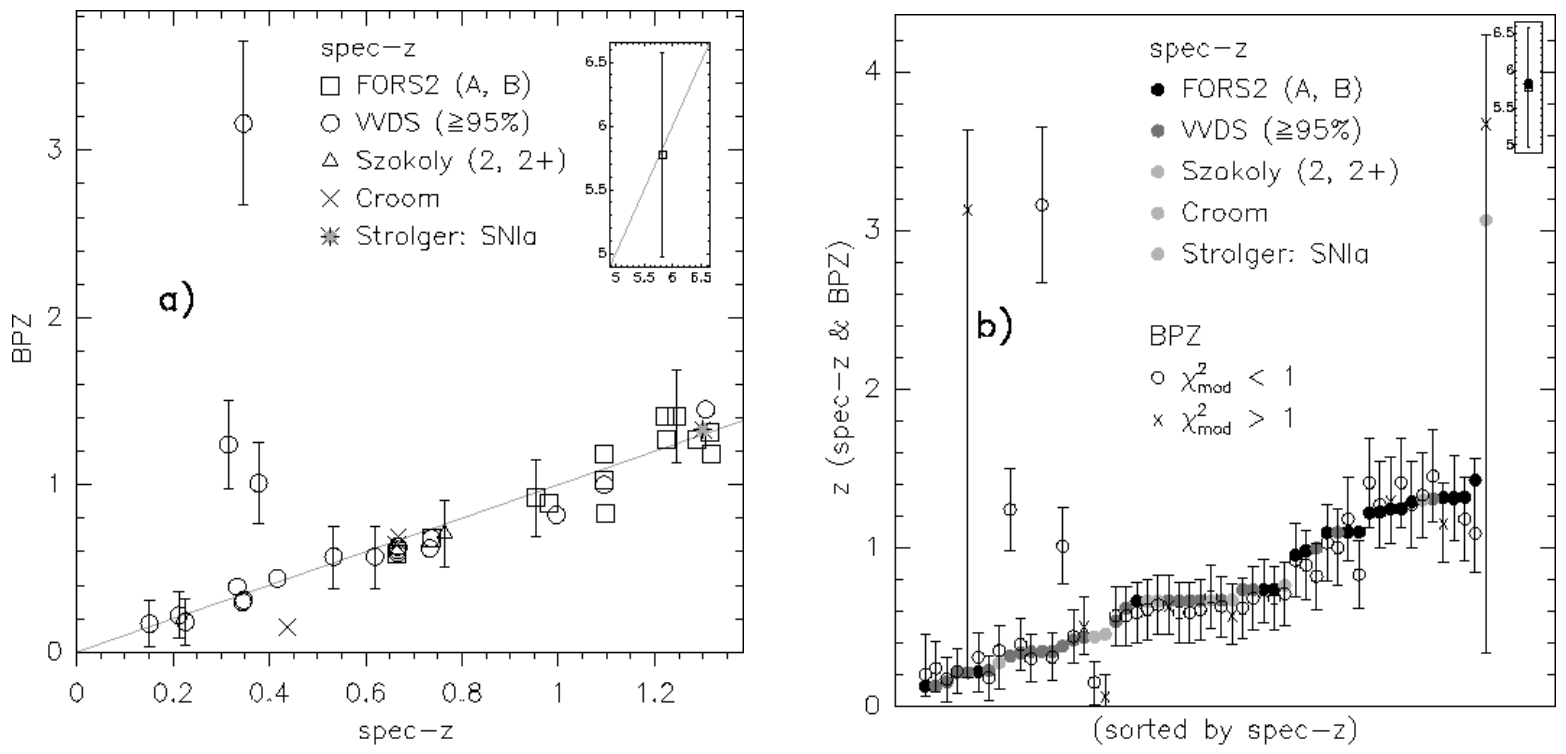

Fig. 16.- Bayesian photometric redshifts compared to the 55 spectroscopic redshifts described in $§ 4.2 .1$. On the left we plot the 41 of those galaxies which have BPZ ODDS $\geq 0.95$ $\& \chi_{\text {mod }}^{2}<1$. The high ODDS values ensure small well-behaved confidence intervals. We plot some of these $95 \%$ confidence intervals, but we suppress most to avoid clutter. We zoom in on those galaxies with lower spec-z and reserve an inset for galaxy \#2225* with $z_{\text {spec }}=5.82$ $\left(z_{b}=5.77 \pm 0.796\right)$. Colors provide the best (or at least most recent) reference for each redshift. On the right we plot the same data, but all redshifts are plotted along the $y$-axis. This plot is less cluttered so we are able to include all 55 galaxies. We are able to plot confidence intervals for all galaxies, so outliers are clearly identified (and we no longer need to restrict ODDS $\geq 0.95$ ). And we are able to include galaxies with $\chi_{\text {mod }}^{2}>1$ by plotting them with a different symbol (an " $\mathrm{x}$ "). To be clear, the $x$-axis is not to scale. It merely serves to spread galaxies across the plot and sort them according to spec-z. 
Wolf et al. (2004) identify a new galaxy cluster at $z \sim 0.15$ within the wider CDF-S field. We are unable to confirm this overdensity in our catalog ( $\$ 4.4)$. We cannot even confirm the redshifts of the $z_{C O M B O-17} \sim 0.15$ galaxies within the UDF. All of these galaxies are faint ( $R \geq 23.5$ ), and BPZ reassigns most of them to $z>1.4$, or outside the redshift range modeled by COMBO-17 (Fig. 18a). The BPZ results are presumably more reliable than COMBO-17 at these faint magnitudes. However Wolf et al. (2004) observe the $z_{C O M B O-17} \sim 0.15$ cluster even in their brightest $R<21$ galaxy sample, which we are in no position to question.

While we are unable to confirm any overdensity at $z \sim 0.15$, we do support COMBO-17's detection of the known overdensity at $z \sim 0.67$ (see discussion and references in $§ 4.4$ )

\subsection{BPZ Histogram}

For each galaxy, BPZ returns a full probability distribution $P(z, t)$ (a function of redshift and type). (The new version of BPZ also returns a catalog summarizing the redshift, width, and ODDS of the three highest peaks.) Fig. 19 shows an example of $P(z)$ for two galaxies, demonstrating how NIC3 photometry helps constrain the fit to a single redshift.

By adding the probability histograms $P(z)$ of individual galaxies, we obtain the redshift probability histogram of the UDF (Fig. 20). Attempting to construct a histogram by binning $z_{b}$ (each galaxy's best fit; the peak of $P(z)$ ) yields a fairly different shape. Fig. 20 only includes galaxies that are detected at the $10-\sigma$ level in at least one filter or detection image. And to exclude stars, we discard all objects with SExtractor stellarity $\geq 0.8$ in the $i^{\prime}$ band image. This eliminates all of the obvious stars (those with diffraction spikes) without removing any obvious high redshift candidates (which may also appear to be pointlike). (All of our best $z \geq 6$ candidate galaxies (Table 10) have stellarity $\leq 0.72$.)

We do not need to discard galaxies based on the BPZ output parameter ODDS. ODDS measures the reliability of each galaxy's most likely redshift $z_{b}$. A galaxy with high ODDS has $P(z)$ with a narrow single peak. Multiple and/or broad peaks yield low ODDS. But all shapes of $P(z)$ will be reflected accurately in our histogram which is a sum of galaxies' $P(z)$ 's.

While we don't need to eliminate galaxies based on low ODDS values, we would like to eliminate those galaxies with ill-fitting SEDs. If BPZ was not able to fit a galaxy well to an SED, then the resulting redshift is probably not accurate. BPZ does return a $\chi^{2}$ goodness-offit value for each galaxy. But low $\chi^{2}$ values (indicating good fits) do not guarantee reliable redshifts. In fact, the opposite was shown to be true in Fig. 8 in the original BPZ paper (Benítez 2000). Galaxies with high $\chi^{2}$ (poor fits) actually have very reliable redshifts. 
The reason for this apparent paradox is that all galaxies with high $\chi^{2}$ are bright galaxies, which have more accurate photometry, in turn yielding more accurate redshifts. Compare the two SED fits shown in Fig. 21. The left panel shows the photometry of a bright galaxy \#6206. The photometric uncertainties are small, typical of bright galaxies. Object \#6206 has been fit to a hybrid El-Sbc SED template at a redshift of $z_{b}=0.92$, which agrees well with the spectroscopic redshift $z_{\text {spec }}=0.95$. The SED fits well but was unable to "thread the needle" of small photometric error bars. Many of the model fluxes are off by several $\sigma$, yielding $\chi^{2}=4.26$.

Faint galaxies on the other hand have much larger error bars, making high values of $\chi^{2}$ almost impossible to achieve, no matter how poor the fit. Object \#7156, only detected at $5-\sigma$, is an extreme example. The photometric uncertainties are so large, that no model fluxes can possibly be off by more than $1-\sigma$, or so. This guarantees a low value for $\chi^{2}$.

Thus we find more reliable redshifts if we restrict our sample to those galaxies with high $\chi^{2}$. Bright galaxies are the only galaxies capable of producing such high values for $\chi^{2}$. But of course high values of $\chi^{2}$ are supposed to indicate poor SED fits.

The solution is to assign an uncertainty to the SED itself. This is represented in Fig. 21 by the blue rectangles. These indicate the model fluxes with uncertainties given by the heights. Our modified version of $\chi^{2}$ is defined as:

$$
\chi_{\text {mod }}^{2}=\sum_{\alpha} \frac{\left(f_{\alpha}-f_{T \alpha}\right)^{2}}{\sigma_{f_{\alpha}}^{2}+\sigma_{f_{T}}^{2}} / \text { d.o.f. }
$$

where $f_{\alpha} \& \sigma_{f_{\alpha}}$ are the observed fluxes and flux errors and $f_{T \alpha}$ are the model fluxes, normalized to fit the observed fluxes. $\sigma_{f_{T}}$ serves as our model flux errors, and we have rather arbitrarily assigned $\sigma_{f_{T}}=\max _{\alpha}\left(f_{T \alpha}\right) / 15$. In other words, $\sigma_{f_{T}}=1 / 15$ on a scale where the highest model flux for a given fit is normalized $f_{T \alpha} \equiv 1$. This "model thickness" dominates over the small flux errors of bright galaxies, yielding a more realistic measure of the goodness of fit. To obtain a "reduced" $\chi^{2}$, we divide by the number of degrees of freedom d.o.f. $=$ \# filters observed -3 . (3 is the number of fit parameters: $z_{b}, t_{b}, a$, or redshift, template, and amplitude. If the object was observed in fewer than 4 filters, we set d.o.f. $=1$.) We now find that object \#6206 is fit almost perfectly by the $z_{b}=0.92$ SED, with $\chi_{\text {mod }}^{2}=0.03$.

$\chi_{\text {mod }}^{2}<1$ roughly corresponds to all model fluxes fitting the observed fluxes within their error bars. By eye, we confirm that galaxies with $\chi_{m o d}^{2}>1$ generally have ill-fitting SEDs. In Fig. 22 we replot our redshift probability histogram but this time only for those galaxies with $\chi_{\text {mod }}^{2}<1$. 
Fig. 23 again replots our redshift probability histogram (Figs. 20 and 22), this time plotting each magnitude range individually. We observe that the peak of the histogram shifts to higher redshift for fainter galaxies. And Fig. 24 breaks our histogram down by spectral type.

Another useful relation is median redshift vs. limiting magnitude (Fig. 25 and Table 9). For each magnitude cut $\left(i^{\prime}<27\right.$, for example), we obtain the redshift probability histogram and find its median. Clustering within the UDF (for example at $z=0.67$, see $\$ 4.4$ ) may affect these results. Of course, whenever possible we encourage the use of photometric redshifts rather than relying on this redshift-magnitude relation. 

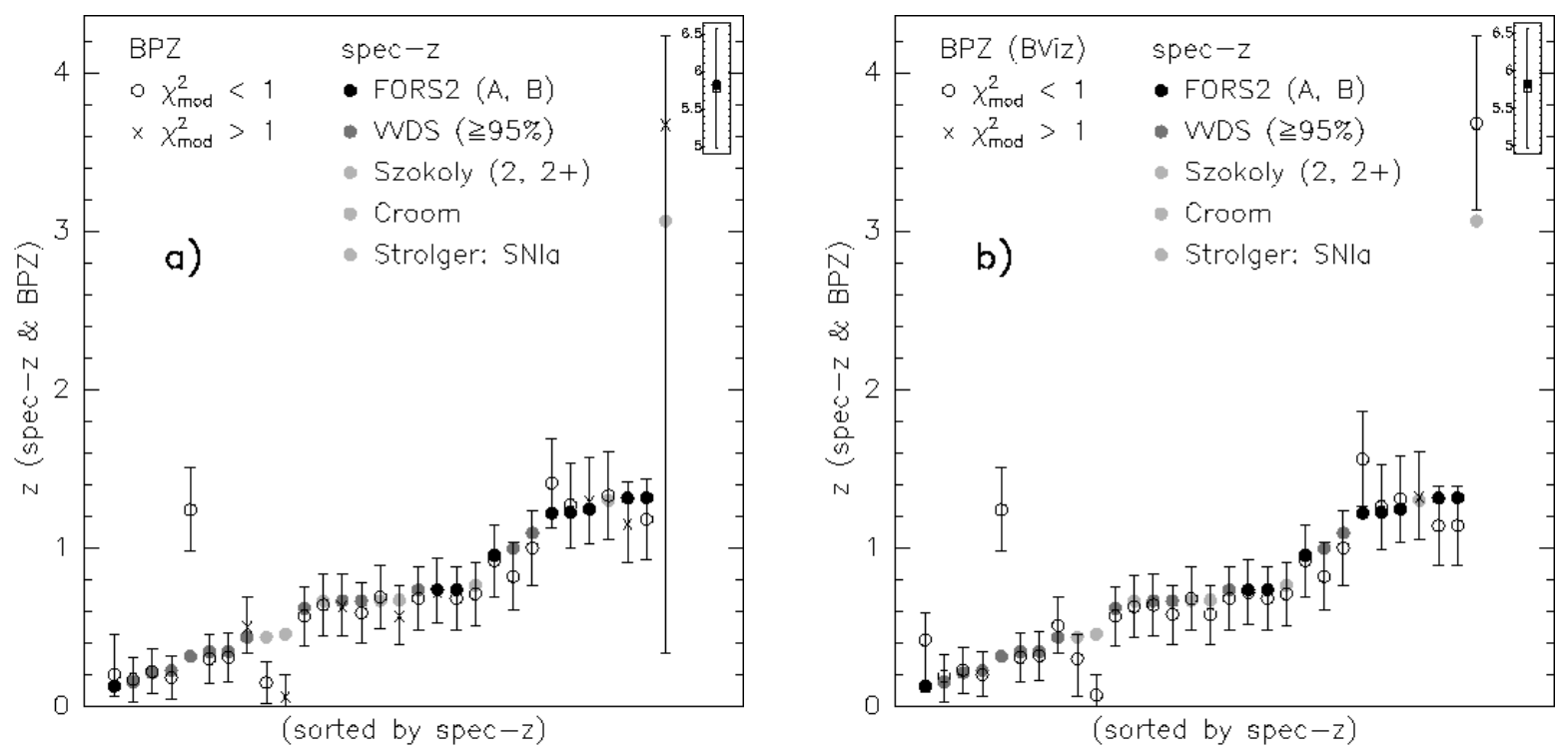

Fig. 17. - Left: same as Fig. 16b, but only for the 31 galaxies within the NIC3 FOV. Right: BPZ results obtained using $B V i^{\prime} z^{\prime}$ filters only. The results degrade very little when the NIC3 filters are omitted, as most of these galaxies are relatively bright (with high signal-to-noise) in $B V i^{\prime} z^{\prime}$. 

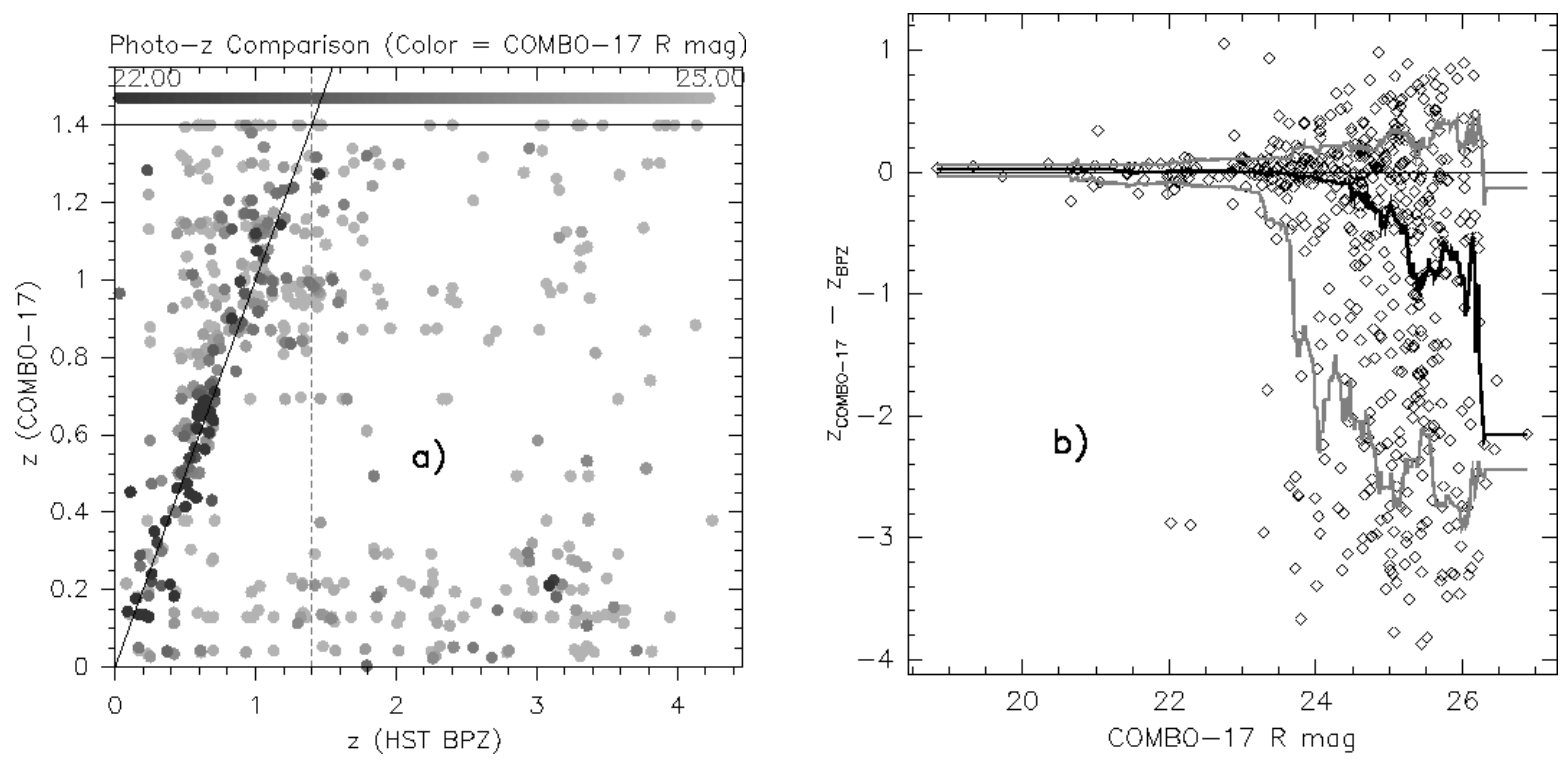

Fig. 18. - Left: Photometric redshifts from COMBO-17 plotted against our Bayesian photometric redshifts. We plot only those galaxies with ODDS $\geq 0.95$ and $\chi_{\text {mod }}^{2}<1$. Colors represent $R$ magnitudes in COMBO-17. COMBO-17 claims to yield reliable redshifts for $R \lesssim 24$, and then only attempts to model redshifts of $z<1.4$. Note that many of the galaxies with COMBO $z \sim 0.13$ have been reassigned BPZ $>1.4$. All but two of these are faint $(R>23.5)$. Right: The same data points replotted as the difference between our redshifts and those obtained by COMBO-17 versus COMBO-17 $R$ magnitude. The inner red line is a moving average (median) of 100 galaxies (or as few as 10 at the edges), while the outer magenta lines contain $68 \%(1-\sigma)$ of the galaxies. The relationship is very good for $R \lesssim 23.7$ and especially tight for $R \lesssim 23$ galaxies, with the notable exception of two outliers with $z_{b} \sim 3$. At fainter magnitudes, a significant fraction of the COMBO-17 redshifts deviate far from our values. 

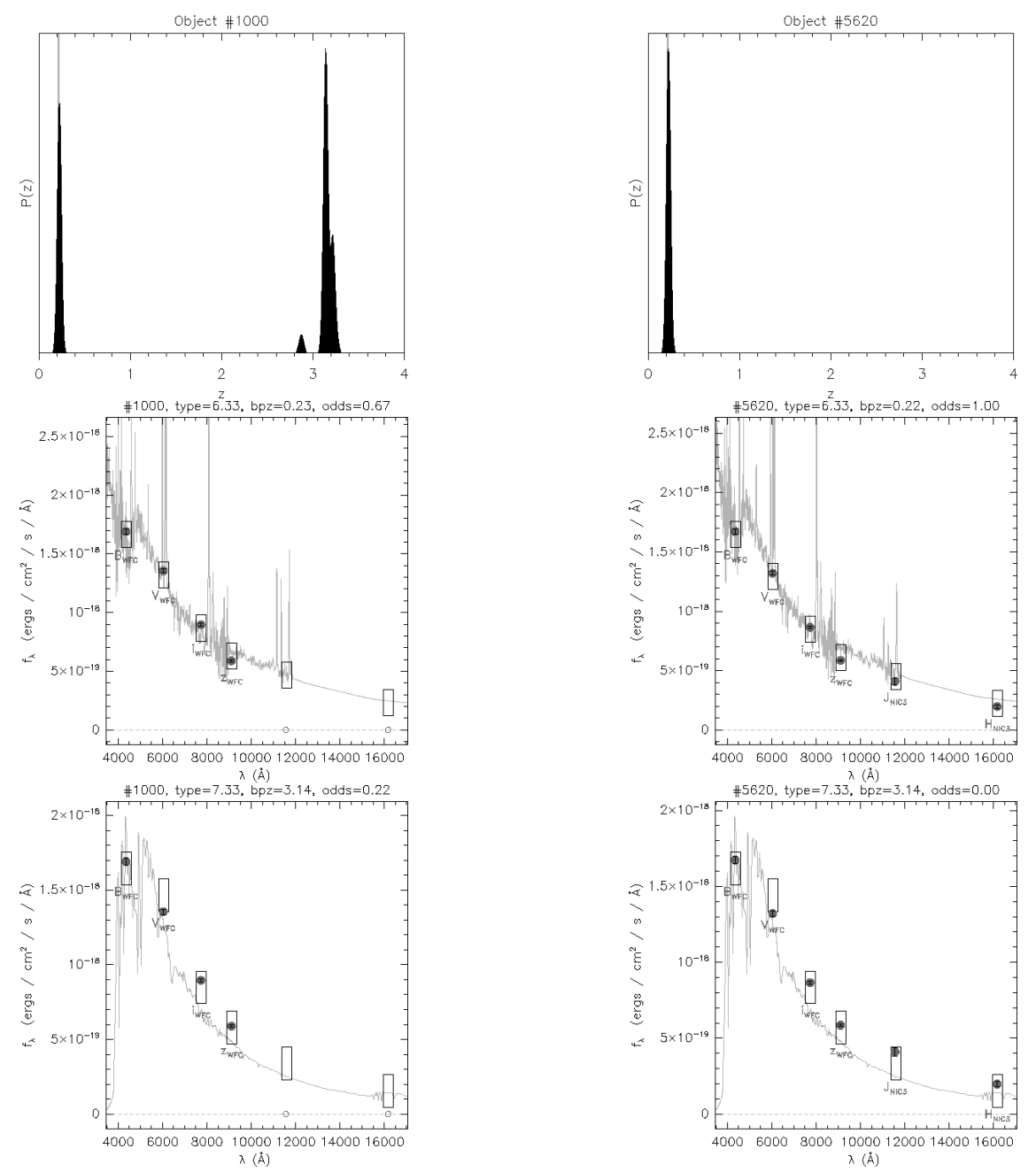

Fig. 19. - Two starburst galaxies $\left(\# 1000 \&\right.$ \&5620) at a spectroscopic redshift of $z_{\text {spec }}=$ 0.21. BPZ returns several possible redshifts for galaxy \#1000. Four peaks are visible in the probability histogram (top left), each corresponding to a different SED fit. The highest peak corresponds to a SB2-25Myr hybrid SED template at $z \sim 0.22$ (middle left). BPZ is $67 \%$ certain (ODDS $=0.67$ ) that this is the correct fit. The second highest peak is a $25 \mathrm{Myr}-5 \mathrm{Myr}$ hybrid at $z \sim 3.14$ (bottom left), which also yields a reasonable fit to the observed $B V i^{\prime} z^{\prime}$ photometry. But because this fit is slightly worse, it earns lower ODDS: a $22 \%$ chance of being the correct fit. Meanwhile, galaxy \#5620 is within the NIC3 FOV. BPZ assigns \#5620 a single redshift peak at the correct redshift (top right). The NIC3 photometry helps constrain the SED fit (middle right). A 25Myr-5Myr SED at $z \sim 3.14$ (bottom right) yields a significantly poorer fit, essentially ruling it out. 

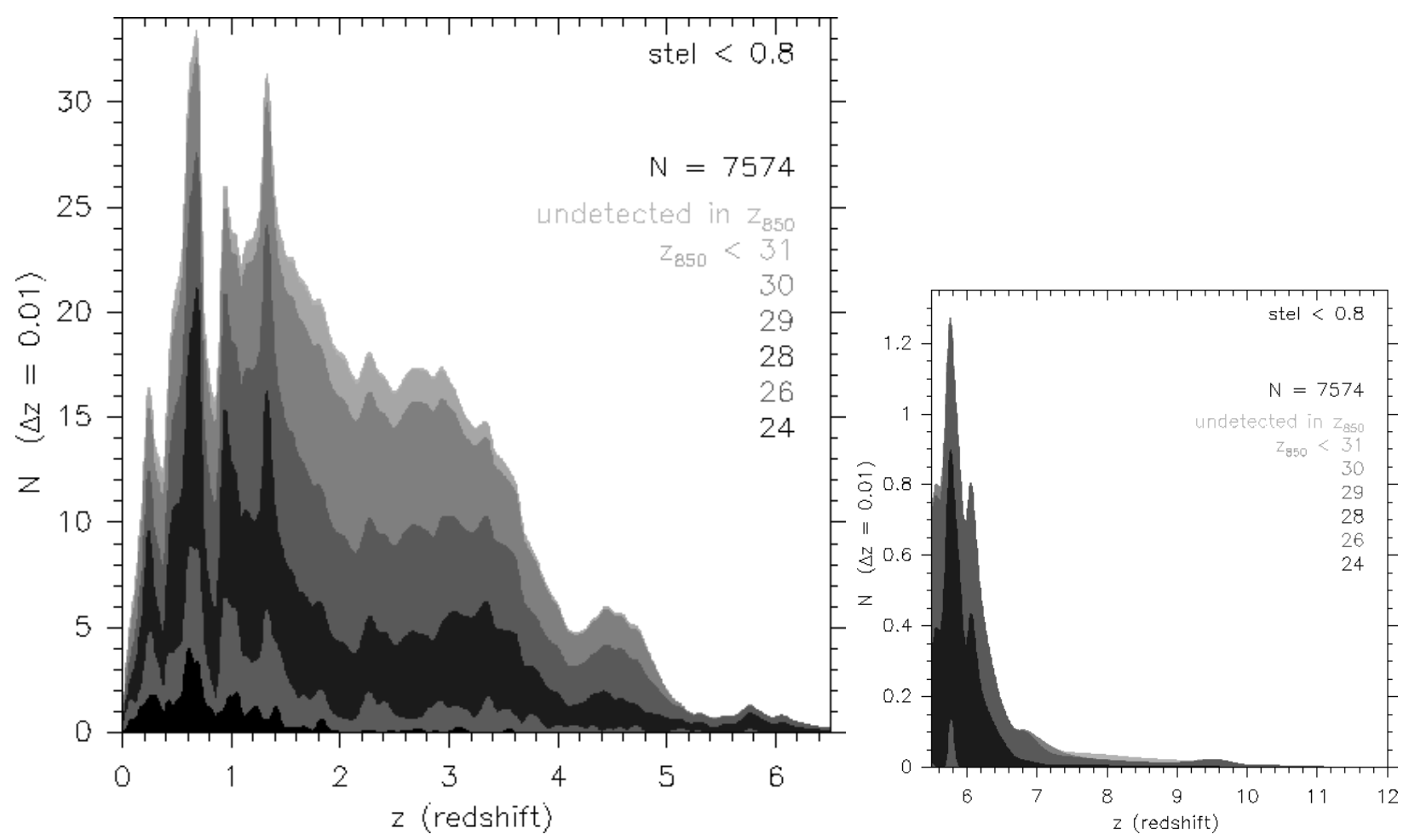

Fig. 20. - BPZ probability histogram obtained by adding the redshift probability distributions $(P(z))$ for 7,574 galaxies. These galaxies have been detected at the $10-\sigma$ level in at least one filter or detection image and have stellarity $<0.8$ in the $i^{\prime}$-band image. We have also excluded galaxies with particularly egregious SED fits: theoretical fluxes of zero or infinite. The redshift interval is 0.01. $z^{\prime}$-band magnitude contours are plotted within the histogram at irregular intervals: undetected in $z^{\prime}$, then $z^{\prime}<31,30,29,28,26,24$. We plot two different redshift ranges: $0<z<6.5$ and $5.5<z<12$. A single galaxy is responsible for the peak at $z_{b}=9.58$ ( see $\left.\S 4.5\right)$. 

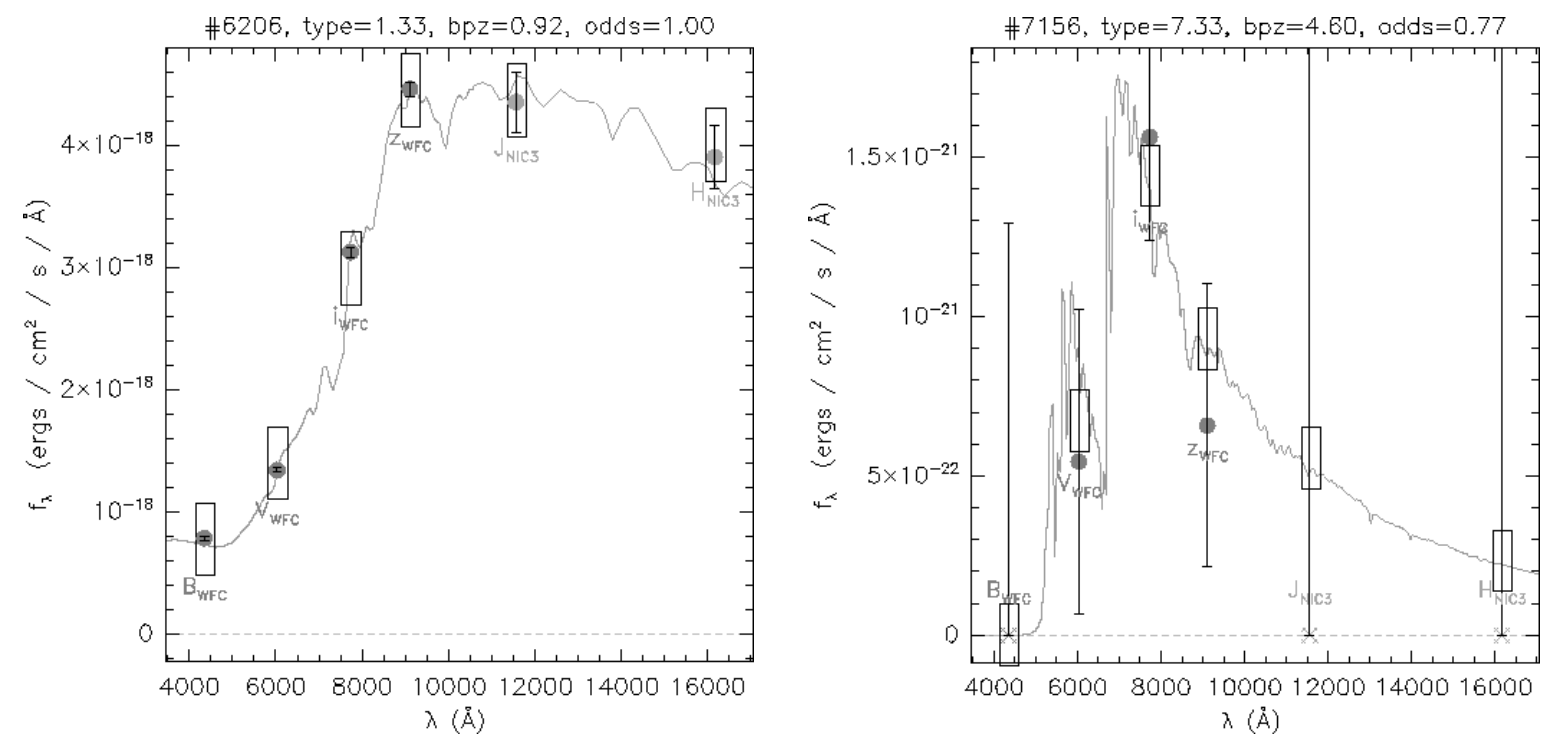

Fig. 21. - SED fits to a bright galaxy (left) and a faint galaxy (right). Bright galaxies yield more accurate photometric redshifts, but the small photometric uncertainties almost ensure a high value of $\chi^{2}$. For this fit, $\chi^{2}=4.27$, even though the photometric redshift $z_{b}=0.92$ matches the spectroscopic redshift $z_{\text {spec }}=0.95$ very well. Meanwhile, the huge photometric uncertainties of object \#7156 (only detected at 5- $\sigma$ ) guarantee a much lower value of $\chi^{2}$. For this fit, we find $\chi^{2}=0.11$. This issue is resolved by assigning uncertainty to the model fluxes, as represented by the heights of the blue rectangles in the plots. The bright galaxy is now a perfect fit with $\chi_{\text {mod }}^{2}=0.03$. The $\chi^{2}$ value of the faint galaxy rises slightly: $\chi_{\text {mod }}^{2}=0.19$. 

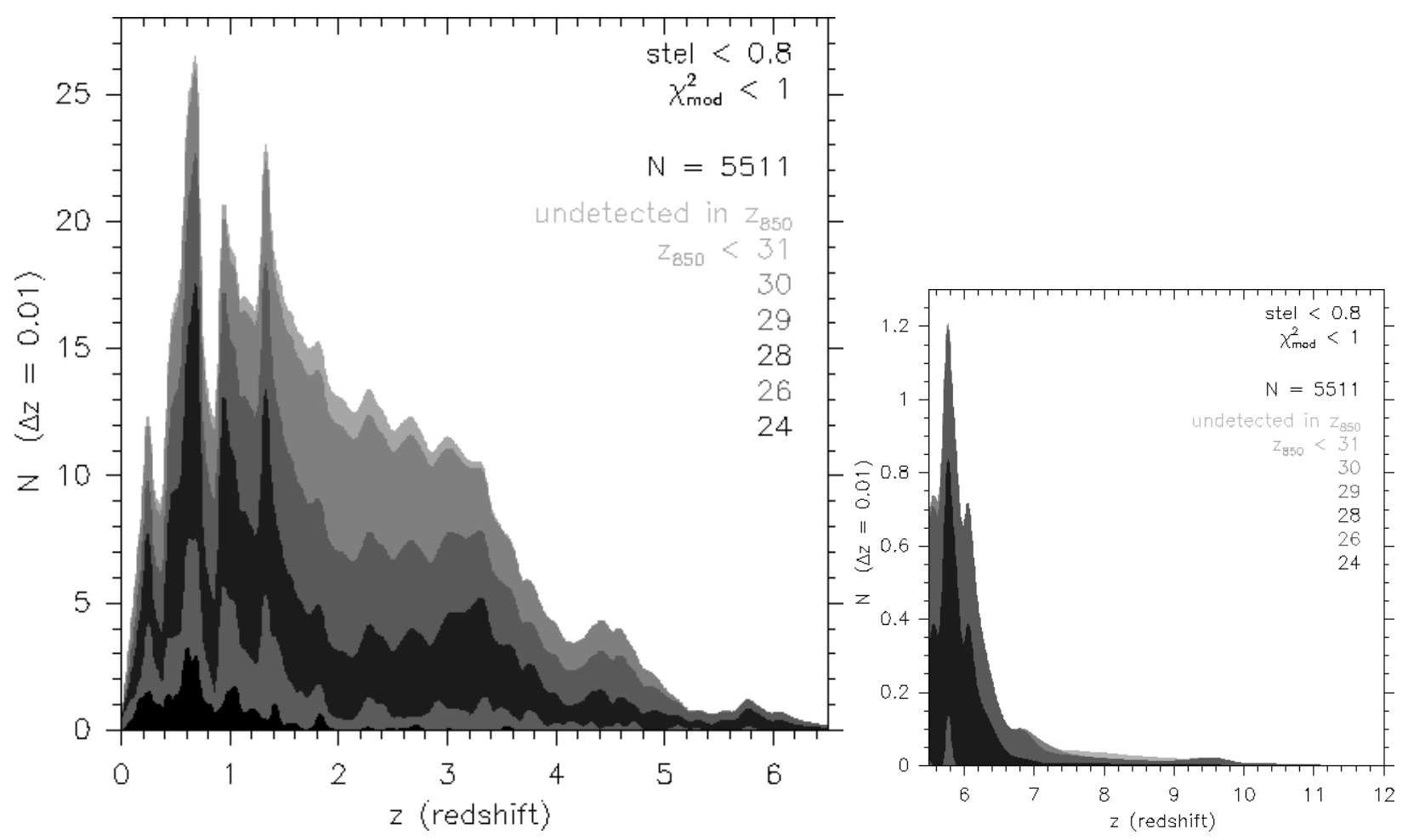

Fig. 22. - A subset of the galaxies plotted in Fig. 20. These 5,511 galaxies all have good SED fits $\left(\chi_{\text {mod }}^{2}<1\right)$. 


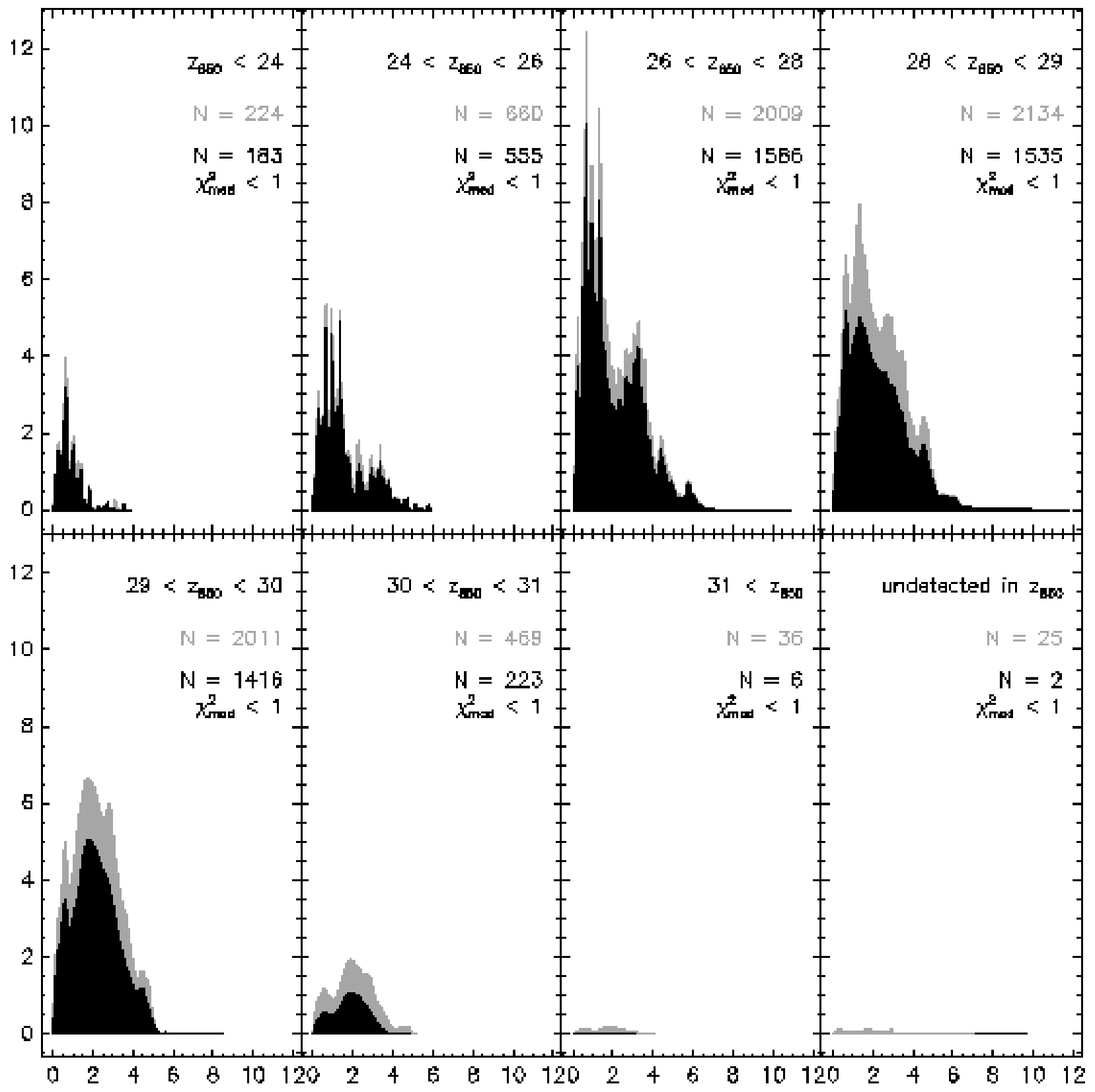

Fig. 23. - Same as Figs. 20 (green) and 22 (black), but broken down into magnitude ranges. The outer green contours plot all galaxies, while the inner black contours plot only those with good SED fits $\left(\chi_{m o d}^{2}<1\right)$. 


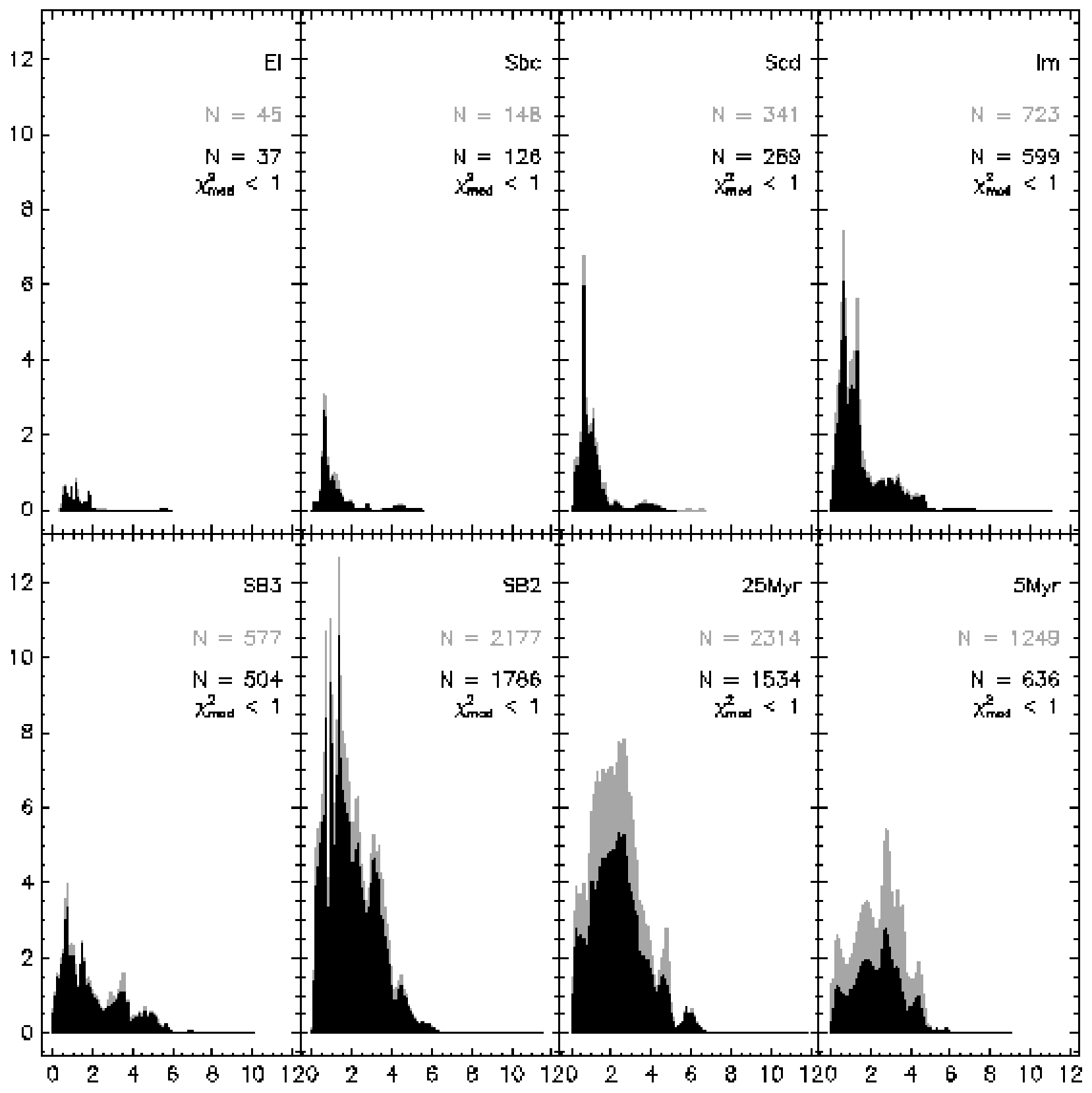

Fig. 24. - Same as Figs. 20 (green) and 22 (black), but broken down into spectral types, as fit by BPZ. Galaxies fit to "interpolated" spectral types are "rounded" to the nearest type. The outer green contours plot all galaxies, while the inner black contours plot only those with good SED fits $\left(\chi_{\text {mod }}^{2}<1\right)$. 


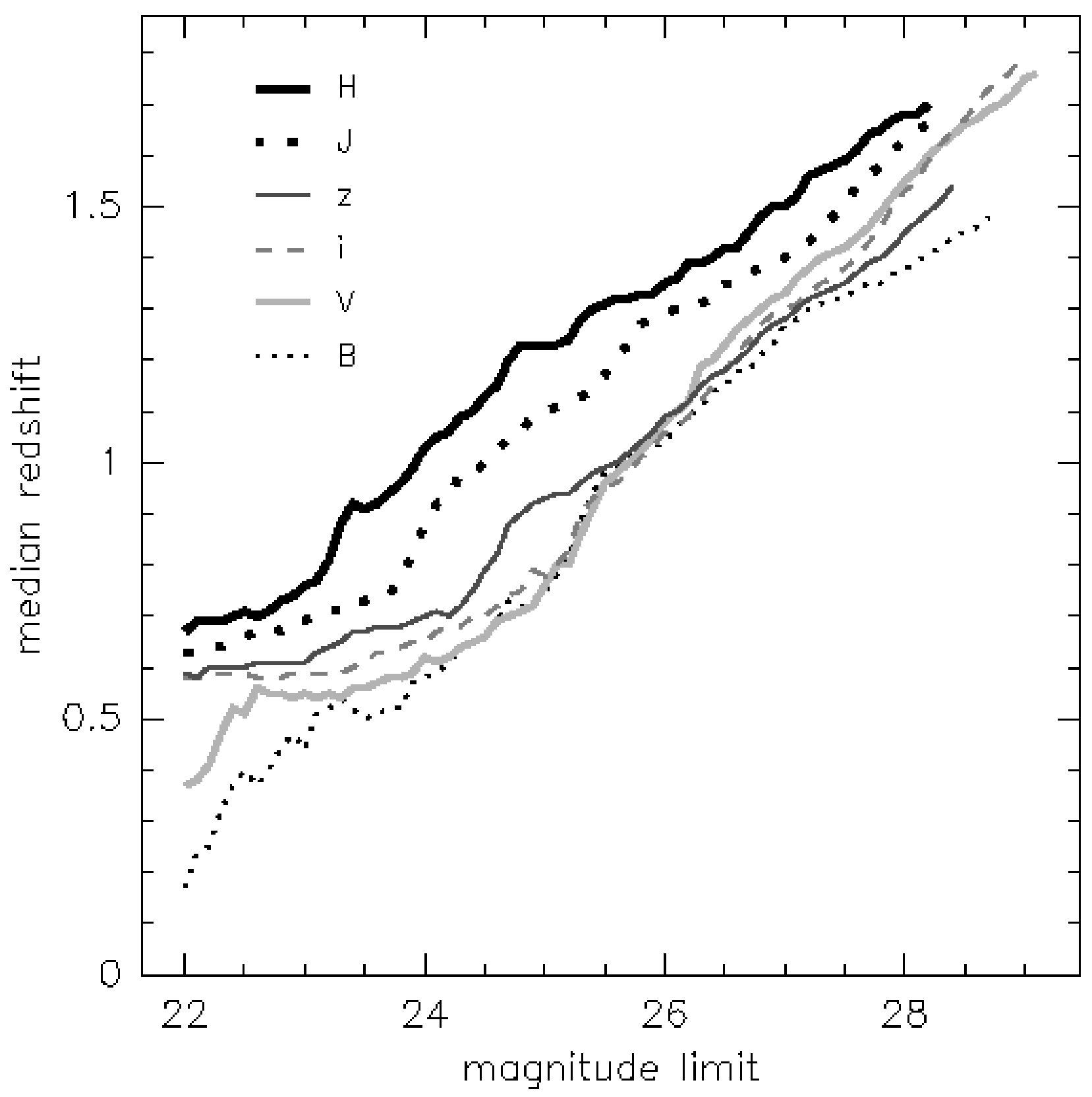

Fig. 25.- Median redshift as a function of limiting magnitude (based on magnitude cuts of our redshift probability histogram). For example, a sample of galaxies complete to $i^{\prime}<27$ will have a median redshift of $z=1.29$. See also Table 9. All curves are truncated at their 10- $\sigma$ completeness limits (e.g., $i^{\prime}=29.01$ ). Offsets have been applied to the NIC3 magnitudes $(\S 4.2 .2)$. 
Table 9. Median Redshifts

\begin{tabular}{ccccccc}
\hline \hline \multirow{2}{*}{$\begin{array}{c}\text { magnitude } \\
\text { limit (AB) }\end{array}$} & $B_{435}$ & $V_{606}$ & $i_{775}^{\prime}$ & $z^{\prime}{ }^{\prime}(z 0$ & $J_{110}{ }^{\mathrm{a}}$ & $H_{160}{ }^{\mathrm{a}}$ \\
\hline 22 & 0.17 & 0.37 & 0.58 & 0.59 & 0.63 & 0.67 \\
23 & 0.44 & 0.55 & 0.59 & 0.61 & 0.69 & 0.76 \\
24 & 0.58 & 0.62 & 0.65 & 0.70 & 0.88 & 1.03 \\
25 & 0.75 & 0.76 & 0.78 & 0.93 & 1.10 & 1.23 \\
26 & 1.04 & 1.08 & 1.06 & 1.09 & 1.29 & 1.35 \\
27 & 1.27 & 1.33 & 1.30 & 1.28 & 1.40 & 1.50 \\
28 & 1.38 & 1.55 & 1.53 & 1.45 & 1.63 & 1.68 \\
29 & 1.51 & 1.75 & 1.79 & 1.70 & 1.74 & 1.74 \\
\hline
\end{tabular}

Note. - Example: a sample of galaxies complete down to a limiting magnitude of $i^{\prime}<27$ will have a median redshift of $z=1.29$. See also Fig. 25 .

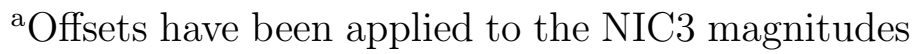
$(\S 4.2 .2)$. 
Fig. 26 examines the 3,783 galaxies $(10-\sigma$, stellarity $<0.8)$ within the NIC3 FOV and how their BPZ results are affected by the availability of NIC3 photometry. Each galaxy's $P(z)$ is compared to that obtained when using only the ACS photometry. For most galaxies, $P(z)$ remains virtually unchanged. This coincident $P(z)$ is plotted as the dark diagonal line. But we also see migration of $P(z)$, for example, from $z \sim 2.5$ to $z \sim 0.3$ and vice versa. This migration pattern is also plotted in greyscale. ${ }^{19}$ The $B V i^{\prime} z^{\prime}$ colors of $z \sim 2.5$ late type galaxies are very similar to the $B V i^{\prime} z^{\prime}$ colors of $z \sim 0.3$ earlier type galaxies. Thus without NIC3 photometry, each of these galaxies is assigned $P(z)$ with two peaks: roughly equal probability of $z \sim 2.5$ and $z \sim 0.3$. But the NIC3 photometry is able to resolve this degeneracy (the nearby earlier type galaxies are brighter in the near IR), reassigning a single redshift (and uncertainty) to each galaxy. In Fig. 26, this appears as symmetric migration between $z \sim 2.5$ and $z \sim 0.3$. We see similar migration between other pairs of redshifts. And at $z>6$ we see a "tail": without NIC3 photometry, all $i^{\prime}$-dropouts (only detected in $z^{\prime}$ ) are simply assigned $z \sim 6$.

\subsubsection{BPZ vs. Maximum Likelihood}

Fig. 27 compares our BPZ results to those obtained from a Maximum Likelihood (ML) approach. Our best fit $z_{b}$ redshifts generally match very well with the ML redshifts, especially for bright galaxies. However, at faint magnitudes, there are a significant number of discrepancies between the two methods. In most of these cases, BPZ realizes its limitations, assigning low ODDS to these galaxies, indicating a broad or multi-peaked redshift distribution $P(z)$. ML methods offer no such measure. And of course, ML does not take advantage of prior knowledge: redshift likelihood as a function of magnitude and type (see Benítez 2000 for details).

Fig. 28 compares redshift histograms of the single values $z_{b}$ and $z_{M L}$ for each galaxy. The $z_{b}$ histogram retains much of the shape of our full probability distribution histogram (Fig. 20), although some differences are apparent. The $z_{M L}$ histogram is fairly similar for $z^{\prime}<28$ galaxies, but markedly different for fainter galaxies.

Most bright $\left(i^{\prime}<26\right)$ galaxies are well defined by their single value redshifts $z_{b}$. But fainter galaxies tend to have broader probability distributions $P(z)$ (i.e., low ODDS). Fig. 29a demonstrates this trend. Fig. 29b replots the same histograms versus redshift $z_{b}$. The low fraction of high ODDS galaxies in the $z_{b}<4$ redshift bins is simply due to the abundance

\footnotetext{
${ }^{19}$ The migration is given by the matrix $A\left[z, z_{A C S}\right]=\sqrt{d P_{z}(z) \times d P_{z A C S}\left(z_{A C S}\right)}$, where $d P_{z}(z)$ and $d P_{z A C S}(z)$ are the positive and negative parts respectively of $P(z)-P_{A C S}(z)$.
} 


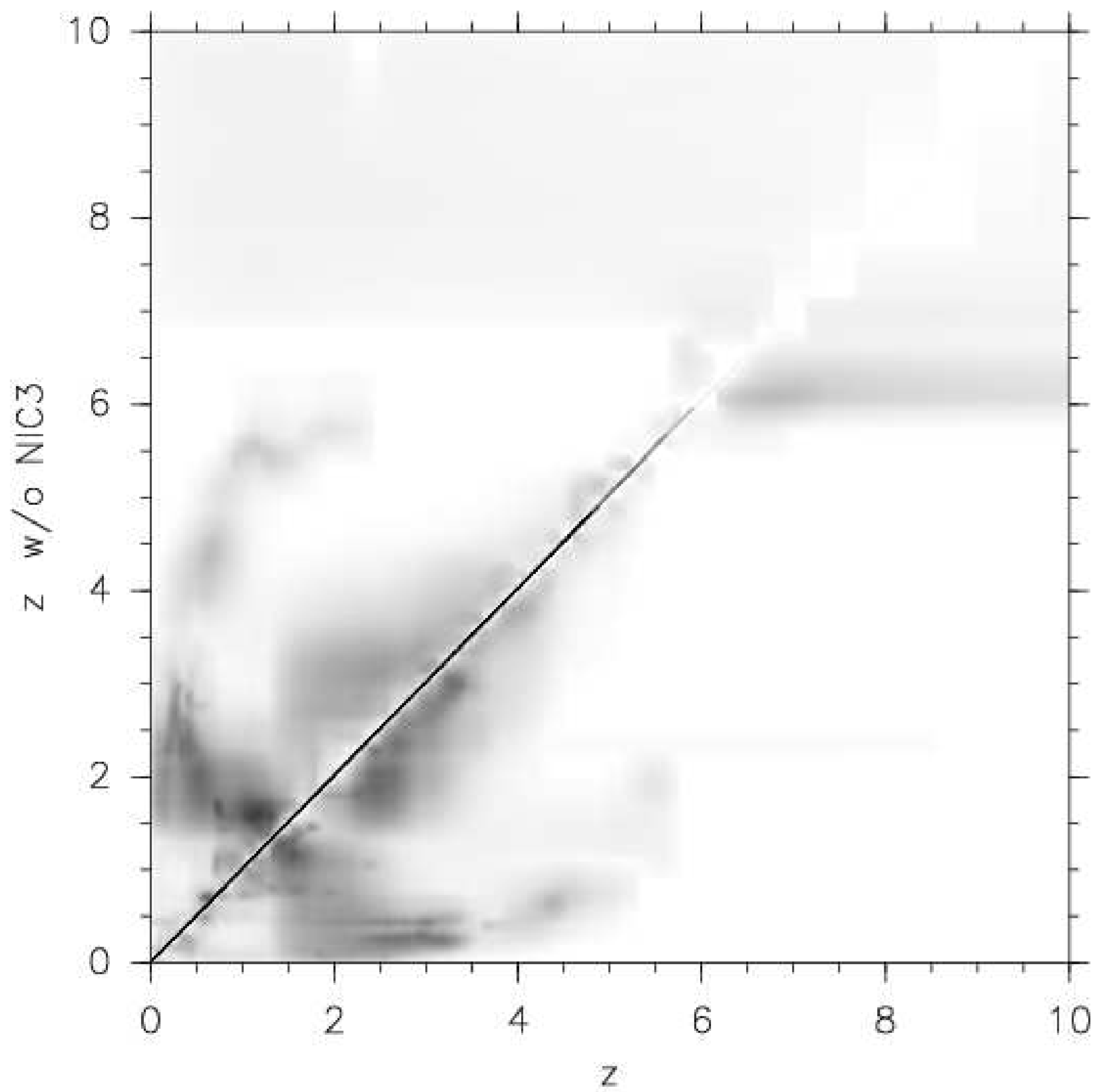

Fig. 26. - BPZ comparison of the 3,783 galaxies $(10-\sigma$, stellarity $<0.8)$ within the NIC3 FOV. BPZ was re-run on these galaxies using only the ACS magnitudes (without NIC3). For each galaxy, we measure the correlation and migration of $P(z)$ between the $B V i^{\prime} z^{\prime}$ and $B V i^{\prime} z^{\prime} J H \mathrm{BPZ}$ runs. The totals are plotted here as greyscale: a clipped square root scale is used to exaggerate the low-level migration. 

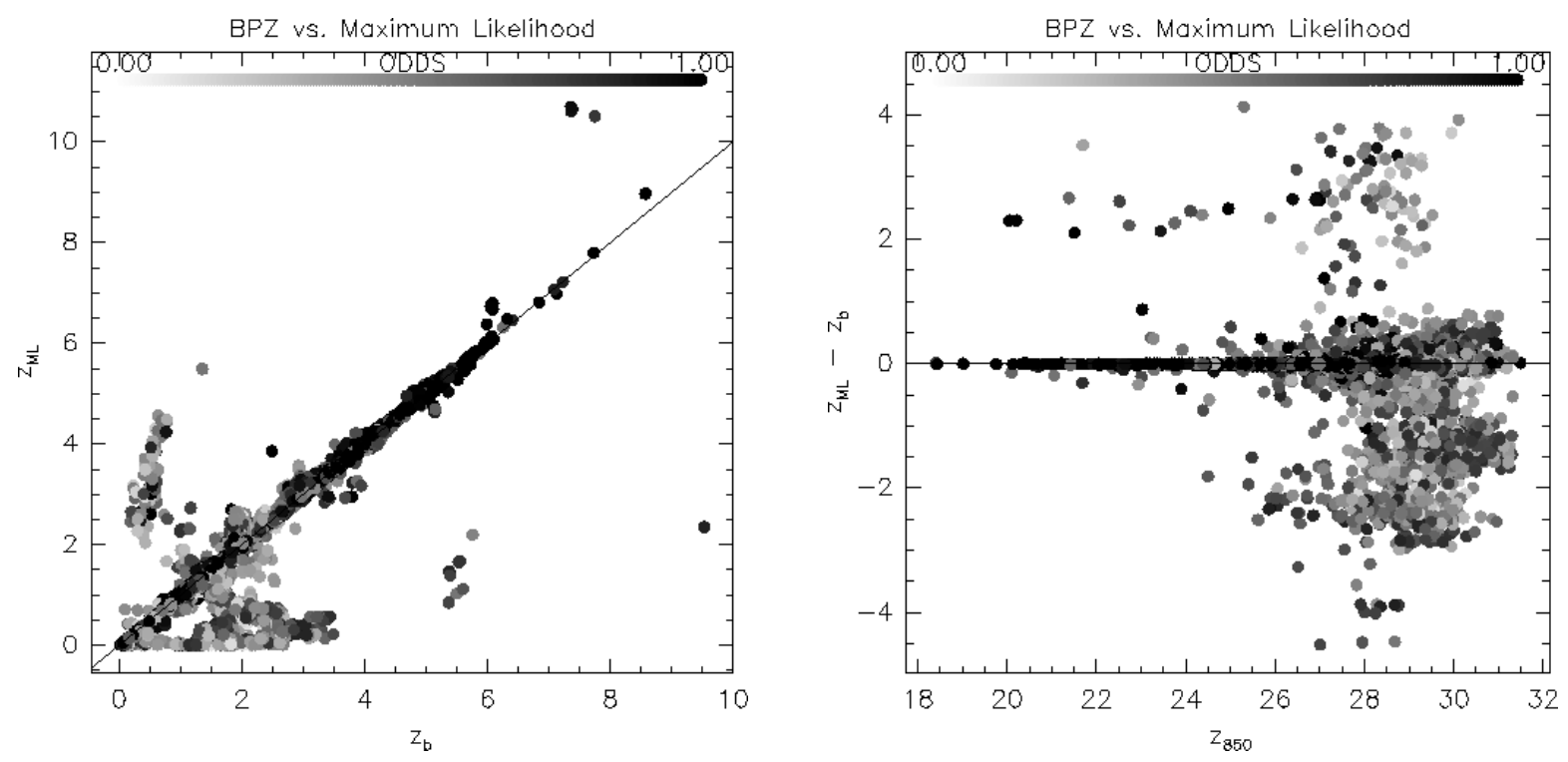

Fig. 27. - BPZ $z_{b}$ vs. Maximum Likelihood redshifts for the $10-\sigma$, stellarity $<0.8$ galaxies. BPZ ODDS values are plotted in greyscale. Trimmed from the plot on the right are galaxies with undetected or "unobserved" (saturated, etc.) $z^{\prime}$-band magnitudes and a few extreme outliers (visible in the plot on the left).
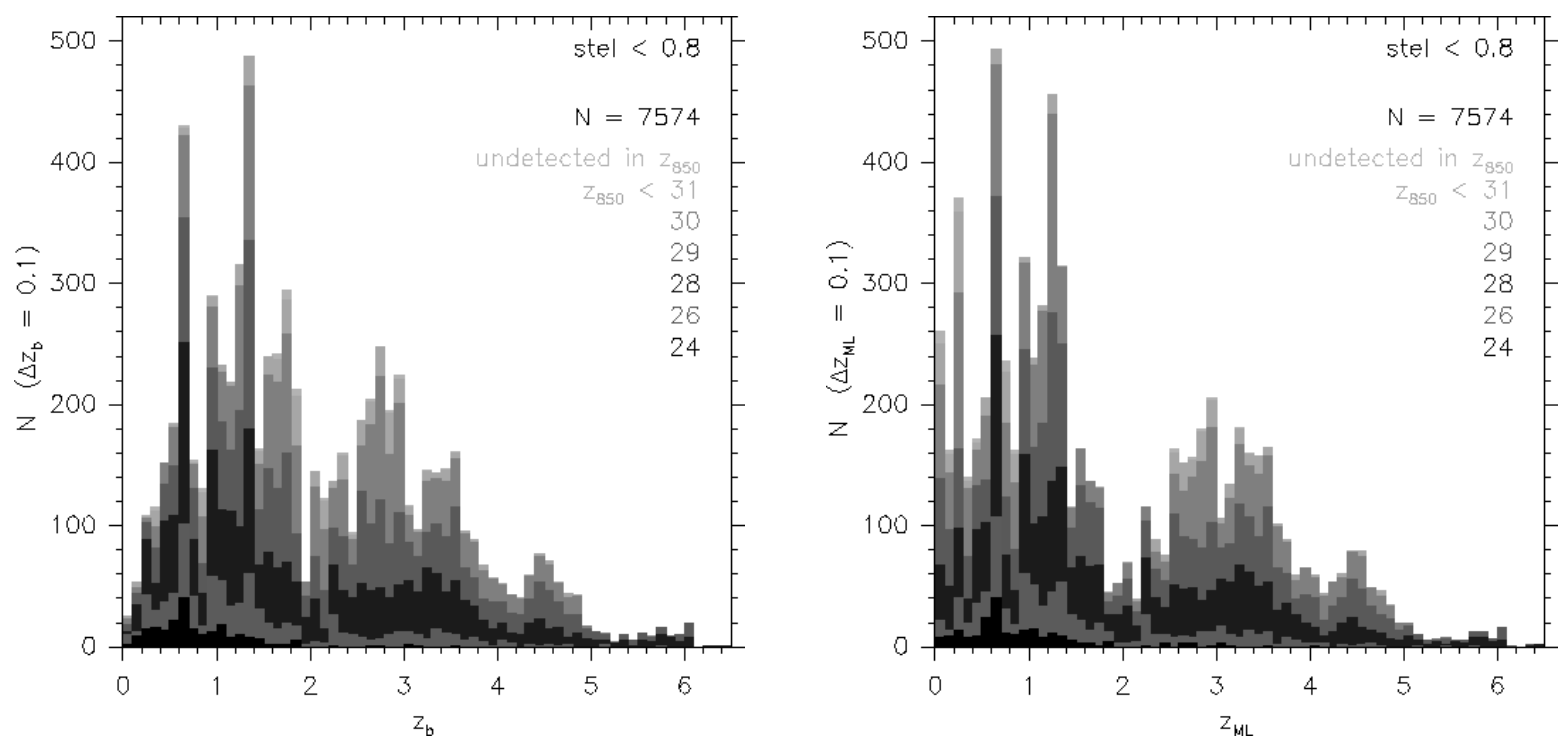

Fig. 28.- Redshift histograms: same as Fig. 20, except binning single value redshifts rather than probability distributions $P(z)$. Left: BPZ best fit redshifts $z_{b}$. Right: Maximum Likelihood redshifts $z_{M L}$. The redshift interval is 0.1 . 
of faint galaxies with uncertain photometry. Galaxies are especially hard to pin down to a single redshift between $2<z_{b}<3$. But note that higher redshift $\left(z_{b}>4\right)$ galaxies are typically dropouts, leaving little doubt about their redshifts.

A plot similar to Fig. 29 can be found in Benítez (2000, Fig. 9). The results for the HDF-N were the same: $i^{\prime}<26$ galaxies were generally well defined by their single value redshifts. $i^{\prime} \gtrsim 27$ galaxies were hard to pin down, and unfortunately they are still hard to pin down in the UDF. There seems to be a strong redshift degeneracy affecting such faint galaxies, probably due to the fact that their average color becomes very blue. This may represent a barrier to the effectiveness of typical photometric redshifts based on a few broad band colors.

\subsection{Clustering}

We detect a strong peak of galaxies at $z \sim 0.67$ (see Fig. 20). Previous studies have identified a group of galaxies at $z=0.67$ and a (denser) cluster of galaxies at $z=0.73$ in the wider CDF-S field (Cimatti et al. 2002, Gilli et al. 2003, Croom et al. 2001, VVDS, FORS2). These galaxies are plotted on the sky in Fig. 30a using the spectroscopic redshifts obtained by VVDS (which is more densely populated than FORS2). The UDF appears to be situated along a clump of the $z=0.67$ group but perhaps within a void of the $z=0.73$ cluster. This claim relies on small number statistics, but our BPZ results do lend some credence to it. When we zoom in on Fig. 20, we find that our redshift distribution exhibits a strong peak at $z \sim 0.67$, but then falls off sharply at $z \sim 0.73$. However it is questionable whether our $\mathrm{BPZ}$ results can be trusted down to this resolution.

Clusters have also been previously identified within the larger field at $z=1.04,1.10$, 1.61, \& 2.57 (Gilli et al. 2003, FORS2). These clusters also seem to avoid the UDF. The right panel of Fig. 30 shows two of these overdensities, again using VVDS. We find no evidence for any of these overdensities within the UDF FOV.

But how sensitive is BPZ to overdensities? In Fig. 31a we plot a histogram of spectroscopic redshifts from the VVDS. Fig. 31b plots this same histogram as it might be observed by BPZ, allowing for redshift uncertainties of $\Delta z=0.04\left(1+z_{\text {spec }}\right)$. (Note that we should expect BPZ to perform this well here, as the VVDS galaxies are all bright: $I<24$.) Note that some of the smaller peaks (e.g., $z=0.13$ ) get washed out by the uncertainty. Redshift peaks only remain at $z \sim 0.7,1.05, \& 0.3$ (in order of decreasing prominence). These broad peaks do show up in the UDF FOV, as we see in Fig. 31c which plots our BPZ probability histogram for similarly bright galaxies: $i^{\prime}<24$. But again, some of the narrower peaks are 


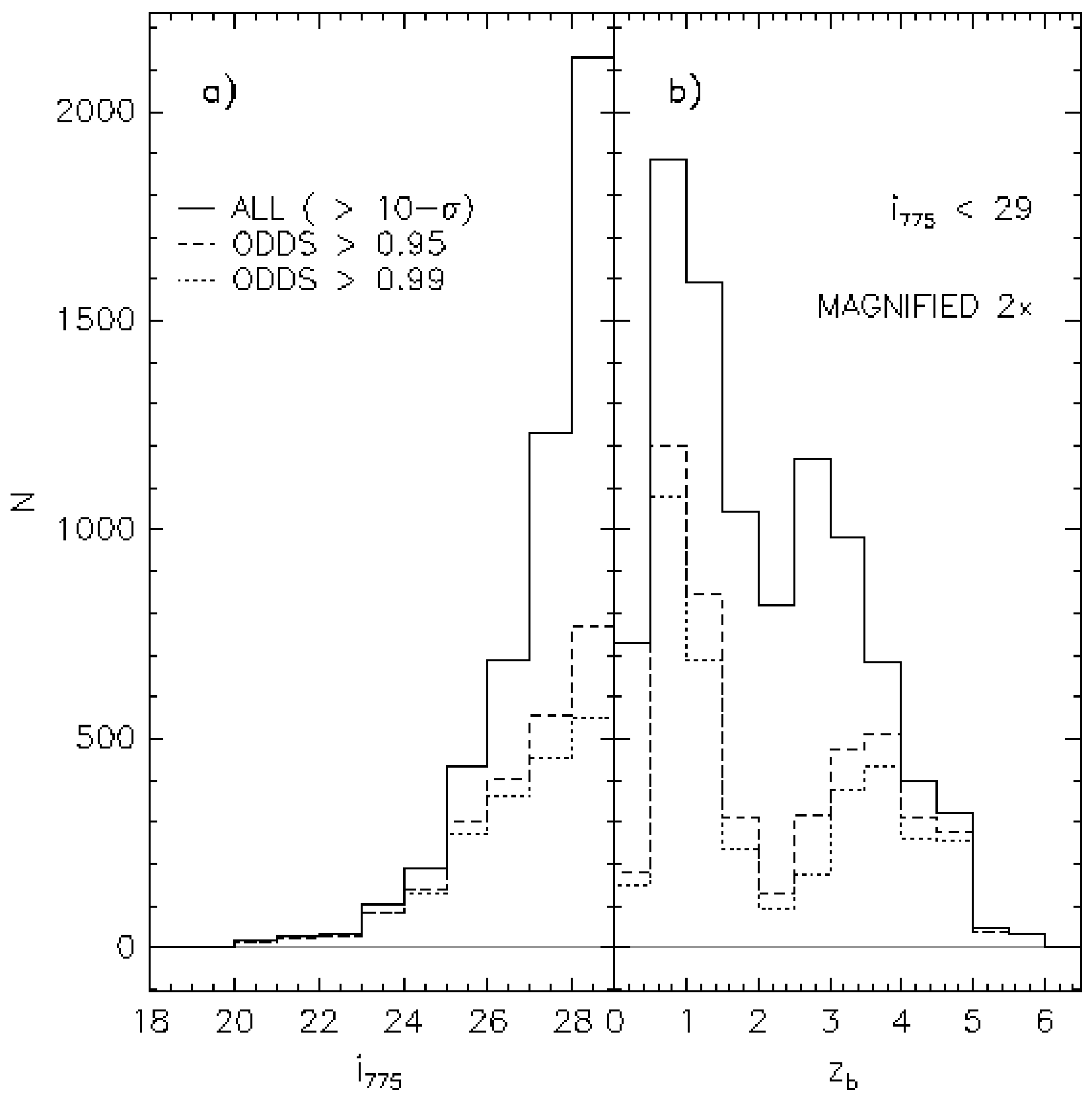

Fig. 29.- Left: Histograms of galaxies $(10-\sigma$, stellarity $<0.8)$ meeting ODDS thresholds of 0.95 and 0.99 as a function of magnitude. Bright galaxies often yield reliable single value redshifts (high ODDS), but fainter galaxies tend to give a broader probability distribution $P(z)$ (low ODDS). Right: Same as left panel, but as a function of redshift and magnified $2 \times$. A similar figure is plotted for the HDF-N in Benítez (2000, Fig. 9). 

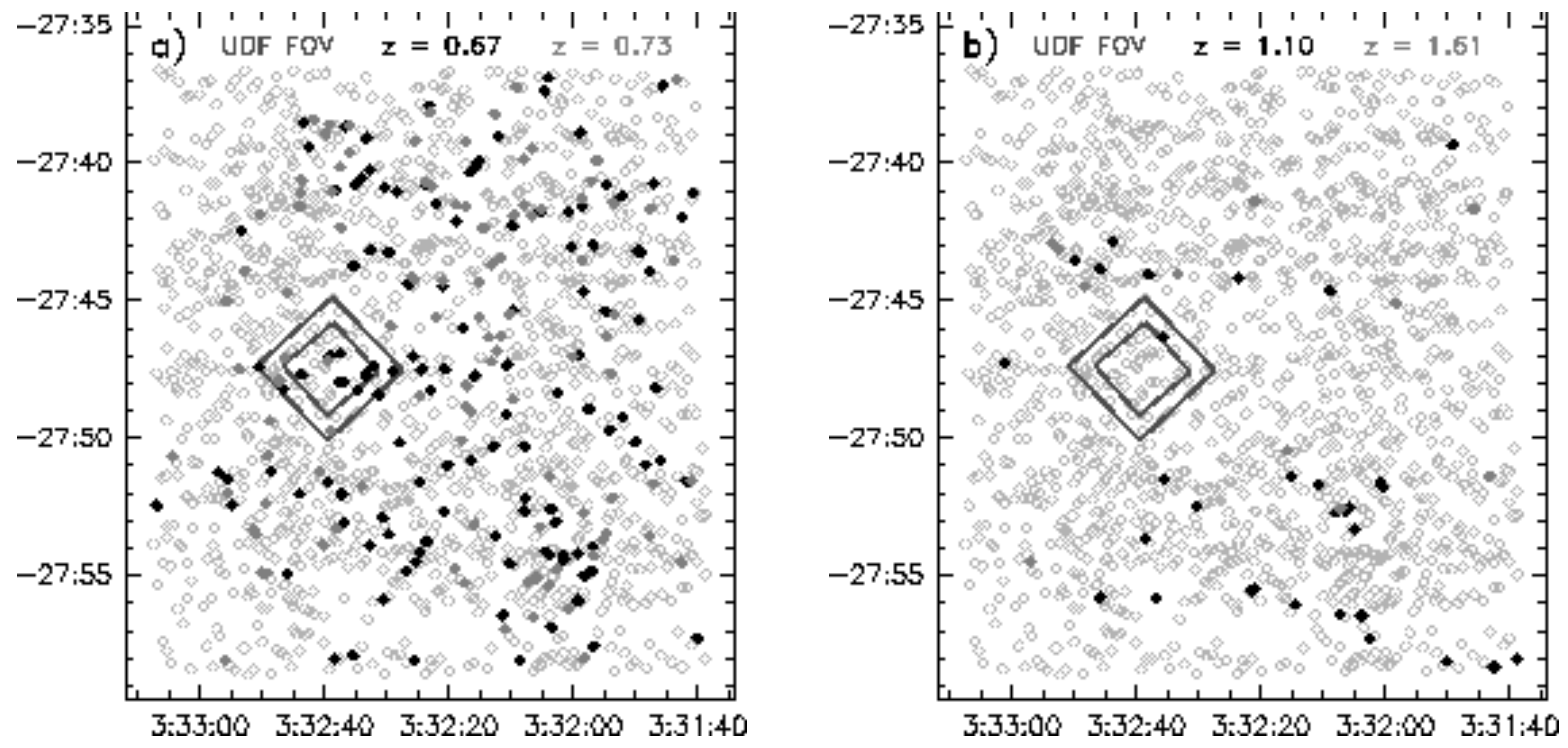

Fig. 30.- Spectroscopic redshifts obtained by VVDS plotted on the sky (RA \& Dec J2000). Left: the $z=0.67$ group $(0.66 \leq z \leq 0.685)$ is plotted in blue. The $z=0.73$ cluster $(0.72 \leq z \leq 0.74)$ is plotted in red. The WFC \& NIC3 FOVs of the UDF are shown in green. Note that the UDF appears to be along a filament of the $z=0.67$ group, but perhaps within a void of the $z=0.73$ cluster. Our BPZ results support this. Right: galaxies with $1.09 \leq z \leq 1.11$ (blue) and $1.60 \leq z \leq 1.64$ (red) cluster in wall-like patterns, deftly avoiding the UDF. 
not apparent.

\subsection{High Redshift Candidates}

Addressing the issue of reionization, previous authors (Bunker et al. 2004; Stanway et al. 2005; Yan \& Windhorst 2004; Bouwens et al. 2005) have identified $i^{\prime}$-dropouts in the UDF. Their catalogs are in good general agreement. When we apply similar criteria to our catalog, we also find decent agreement. The $i^{\prime}$-dropout technique is robust: BPZ is not significantly better at identifying $z \sim 6$ galaxies. (At lower redshifts, photometric redshifts do yield a more complete catalog than Lyman-break techniques.)

There is little disagreement among the various authors about the number of $z \sim 6$ galaxies, and yet these $50+$ objects leave much open to interpretation. For example, a luminosity function must be assumed to address the issue of completeness. There is even some debate surrounding the exact conditions required for reionization (Stiavelli et al. 2004). Sorting out these issues is beyond the scope of this paper and has been addressed in depth elsewhere (e.g., Bouwens et al. 2005). For now we simply present our "best" $z \geq 6$ candidates within the NIC3 FOV (Table 10).

In Paper II, we will return to all of these issues briefly, including detailed comparisons of our $z \sim 6$ results with previous authors. Yet, there is much to be learned at $z<6$. The SFR history of the universe remains fairly uncertain between $3 \lesssim z \lesssim 6$. The UDF provides allows us to probe the $z \sim 4$ luminosity function with a complete sample of galaxies all the way down to $M_{\star}+3$. Thus Paper II will concentrate on the $z<6$ SFR history in the UDF. 

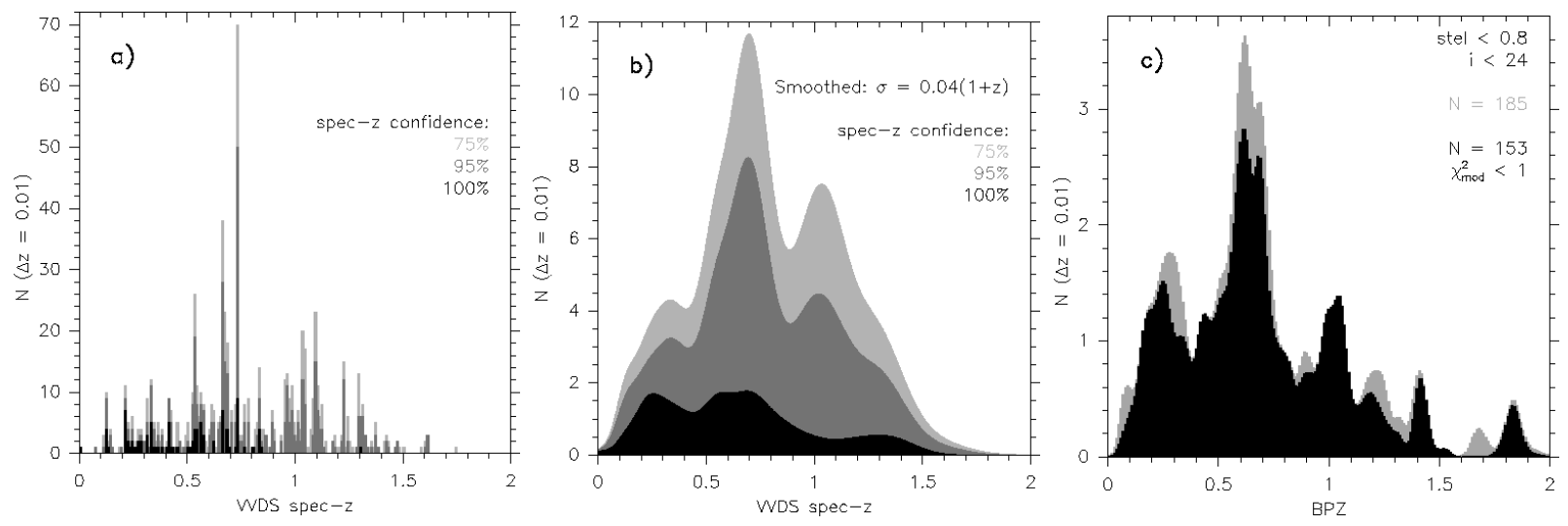

Fig. 31. - Left: Histogram of VVDS spectroscopic redshifts for galaxies within the larger CDF-S field. All galaxies are $I<24$ and have spec-z confidence $\geq 75 \%$. Galaxies at $z>2$ are not plotted. Center: The same histogram smoothed by $\Delta z=0.04(1+z)$, or how it might be observed by BPZ. Right: Our UDF BPZ probability histogram (also cut off at $z<2$ ) for similarly bright galaxies: $i^{\prime}<24$. Note the general good agreement with the center panel. 
Table 10. "Best" High Redshift $\left(z_{b} \geq 6\right)$ Candidates

\begin{tabular}{|c|c|c|c|c|c|c|c|c|c|c|}
\hline \multirow{2}{*}{$\frac{\mathrm{ID}}{32521^{*}}$} & \multicolumn{2}{|c|}{ RA \& DEC (J2000) } & \multirow{2}{*}{$\frac{z_{b}}{6.03 \pm 0.83}$} & \multirow{2}{*}{$\frac{\chi_{\text {mod }}^{2}}{0.01}$} & \multirow{2}{*}{$\frac{B_{435}}{>30.37}$} & \multirow{2}{*}{$\frac{V_{606}}{>30.76}$} & \multirow{2}{*}{$\frac{i^{\prime} 775}{29.31 \pm 0.28}$} & \multirow{2}{*}{$\frac{z^{\prime} 850}{26.83 \pm 0.06}$} & \multirow{2}{*}{$\frac{J_{110}}{26.76 \pm 0.30}$} & \multirow{2}{*}{$\frac{H_{160}}{26.74 \pm 0.33}$} \\
\hline & $03: 32: 36.625$ & $-27: 47: 50.06$ & & & & & & & & \\
\hline $4110^{*}$ & $03: 32: 41.573$ & $-27: 47: 44.24$ & $6.03 \pm 0.83$ & 0.21 & $>30.75$ & $>31.17$ & $29.18 \pm 0.18$ & $26.73 \pm 0.04$ & $26.84 \pm 0.19$ & $26.71 \pm 0.20$ \\
\hline 32042 & 03:32:40.553 & $-27: 48: 02.60$ & $6.03 \pm 0.83$ & 0.45 & $>31.48$ & $>31.91$ & $31.11 \pm 0.47$ & $28.43 \pm 0.09$ & $28.25 \pm 0.70$ & $>28.64$ \\
\hline 31496 & 03:32:39.127 & $-27: 48: 18.47$ & $6.04 \pm 0.83$ & 0.13 & $>31.97$ & $>32.41$ & $31.46 \pm 0.42$ & $28.96 \pm 0.11$ & $28.42 \pm 0.67$ & $>28.26$ \\
\hline 34321 & 03:32:44.701 & $-27: 47: 11.57$ & $6.04 \pm 0.83$ & 0.53 & $30.67 \pm 0.44$ & $>31.87$ & $30.69 \pm 0.36$ & $28.06 \pm 0.07$ & $27.97 \pm 0.28$ & $28.18 \pm 0.34$ \\
\hline 33268 & $03: 32: 34.526$ & $-27: 47: 34.84$ & $6.05 \pm 0.83$ & 0.65 & $>31.75$ & $>32.16$ & $31.24 \pm 0.43$ & $28.59 \pm 0.09$ & $28.01 \pm 0.39$ & $>28.77$ \\
\hline 34942 & $03: 32: 34.575$ & $-27: 46: 58.00$ & $6.05 \pm 0.83$ & 0.75 & $31.21 \pm 0.73$ & $31.19 \pm 0.54$ & $31.56 \pm 0.75$ & $28.27 \pm 0.10$ & $27.99 \pm 0.61$ & $>28.20$ \\
\hline $8033^{*}$ & $03: 32: 36.473$ & $-27: 46: 41.45$ & $6.05 \pm 0.83$ & 0.92 & $>30.63$ & $30.93 \pm 0.72$ & $28.64 \pm 0.13$ & $26.05 \pm 0.02$ & $26.13 \pm 0.14$ & $25.71 \pm 0.14$ \\
\hline 32007 & 03:32:42.797 & $-27: 48: 03.24$ & $6.07 \pm 0.83$ & 0.24 & $>31.59$ & $>32.01$ & $31.16 \pm 0.46$ & $28.16 \pm 0.07$ & $27.80 \pm 0.31$ & $28.22 \pm 0.49$ \\
\hline $7730^{*}$ & $03: 32: 38.282$ & $-27: 46: 17.22$ & $6.08 \pm 0.83$ & 0.28 & $29.79 \pm 0.30$ & $30.48 \pm 0.38$ & $29.84 \pm 0.25$ & $26.67 \pm 0.03$ & $26.39 \pm 0.16$ & $26.25 \pm 0.17$ \\
\hline 35616 & 03:32:37.690 & $-27: 46: 21.57$ & $6.10 \pm 0.84$ & 0.21 & $>31.66$ & $>32.05$ & $>31.93$ & $28.63 \pm 0.11$ & $28.32 \pm 0.64$ & $28.14 \pm 0.67$ \\
\hline $8545^{*}$ & $03: 32: 37.465$ & $-27: 46: 32.67$ & $6.26 \pm 0.85$ & 0.39 & $>30.26$ & $>30.66$ & $>30.54$ & $26.66 \pm 0.06$ & $26.19 \pm 0.12$ & $26.22 \pm 0.13$ \\
\hline $33003^{*}$ & 03:32:35.053 & $-27: 47: 40.18$ & $6.32 \pm 0.86$ & 0.06 & $>31.20$ & $>31.60$ & $31.20 \pm 0.63$ & $27.81 \pm 0.07$ & $27.11 \pm 0.17$ & $26.96 \pm 0.17$ \\
\hline $4050^{*}$ & 03:32:33.427 & $-27: 47: 44.88$ & $6.40 \pm 0.87$ & 0.17 & $30.35 \pm 0.45$ & $31.30 \pm 0.67$ & $29.63 \pm 0.20$ & $27.29 \pm 0.05$ & $26.60 \pm 0.16$ & $26.68 \pm 0.17$ \\
\hline $34987^{*}$ & 03:32:42.560 & $-27: 46: 56.62$ & $6.84 \pm 0.92$ & 0.02 & $>30.90$ & $>31.31$ & $>31.18$ & $28.04 \pm 0.12$ & $26.54 \pm 0.16$ & $26.02 \pm 0.14$ \\
\hline 41066 & $03: 32: 42.558$ & $-27: 47: 31.39$ & $7.13 \pm 0.96$ & 0.04 & $>31.20$ & $>31.62$ & $>31.50$ & $29.53 \pm 0.32$ & $27.51 \pm 0.23$ & $26.76 \pm 0.19$ \\
\hline 41092 & 03:32:38.798 & $-27: 47: 07.11$ & $7.73 \pm 1.03$ & 0.30 & $>31.06$ & $>31.48$ & $>31.37$ & $>30.67$ & $26.81 \pm 0.19$ & $26.66 \pm 0.19$ \\
\hline 41107 & $03: 32: 40.937$ & $-27: 47: 41.83$ & $8.57 \pm 1.12$ & 0.00 & $>30.82$ & $31.09 \pm 0.67$ & $>31.18$ & $>30.35$ & $24.86 \pm 0.16$ & $23.71 \pm 0.18$ \\
\hline
\end{tabular}

Note. - We select here only galaxies detected in multiple filters including at least one NIC3 filter, with ODDS $\geq 0.95$, and with $\chi_{\text {mod }}^{2}<1$. ID numbers below 41000 correspond to B04 \& T04 detections; asterisks (*) indicate that object definitions have been altered (§3.1). Magnitudes are "total" AB magnitudes with isophotal colors: NIC3 magnitudes are corrected to the PSF of the ACS images $(\S 3.3)$. We have also applied offsets of $(J$ : $-0.30 \pm 0.03$, $H:-0.18 \pm 0.04)$ to the NIC3 magnitudes (\$4.2.2). And all of our magnitudes have been corrected for galactic extinction (Table 2). Non-detections (listed, for example, as $>31.05)$ quote the $1-\sigma$ detection limit of the aperture used on the given object. $z_{b}$ gives the peak of the Bayesian photometric redshift distribution $P(z)$, while $\chi_{m o d}^{2}$ measures how poorly the best fitting SED template at $z_{b}$ fits the observed colors. Color images of these objects along with SED fits and more are available at http://adcam.pha.jhu.edu/ ${ }^{\sim} \operatorname{coe} / \mathrm{UDF} / z 6 \mathrm{~g} /$. 


\section{Summary}

We have presented a catalog of photometry, Bayesian photometric redshifts, and morphological parameters for galaxies in the UDF (8,042 of which are detected at 10- $\sigma$ ). Our comprehensive catalog combines $i^{\prime}, z^{\prime}, J+H$, and $B+V+i^{\prime}+z^{\prime}$ detections. To facilitate comparison with catalogs released by B04 and T04, most of our object definitions are taken directly from their segmentation maps. Our robust photometric method corrects the $z^{\prime}, J$, \& $H$ magnitudes for the wider PSFs observed in those bands. NIC3 magnitudes proved too faint relative to ACS magnitudes of galaxies with known spectroscopic redshift. To correct for this, magnitude offsets $(J:-0.30 \pm 0.03, H:-0.18 \pm 0.04)$ were applied to our catalog. Part of these offsets $(J: 0.08, H: 0.09)$ have since been attributed to a slight miscalibration of the filter response curves used to produce the NICMOS Treasury catalog (Thompson et al. 2006). While the rest of the offsets appear to stem from a count-rate dependent non-linearity in NICMOS (de Jong et al. 2006b).

The UDF reveals a large population of faint blue galaxies (presumably young starbursts), bluer than those observed in the original Hubble Deep Fields (HDF). We present a redshift histogram derived from full BPZ probability distributions. A strong peak is observed at $z \sim 0.67$, corresponding to a known group of galaxies in the wider CDF-S. Our results and software packages are available at http://adcam.pha.jhu.edu/ coe/UDF/.

We are indebted to Steven Beckwith, Rodger Thompson, their teams, and to everyone who has worked to provide the astronomical community with this tremendous dataset. We would also like to thank Roelof de Jong, Rodger Thompson, Bahram Mobasher, Louis Eddie Bergeron, and Adam Riess for numerous valuable conversations regarding the UDF NIC3 calibration. And we thank Stephen Gwyn for discussing his independent derivation of identical empirical offsets. We especially thank the referee for helpful comments that have improved the paper. ACS was developed under NASA contract NAS 5-32865, and this research is supported by NASA grant NAG5-7697. We are grateful for an equipment grant from the Sun Microsystems, Inc. This work has also been supported by the European Commission Marie Curie International Reintegration Grant 017288-BPZ and the PNAYA grant AYA2005-09413-C02. 


\section{REFERENCES}

Abraham, R. G., van den Bergh, S., Glazebrook, K., Ellis, R. S., Santiago, B. X., Surma, P., \& Griffiths, R. E. 1996, ApJS, 107, 1

Andredakis, Y. C., Peletier, R. F., \& Balcells, M. 1995, MNRAS, 275, 874

Beckwith, S., Somerville, R., Stiavelli, M. 2003, STScI Newsletter vol 20 issue 04

Benítez, N., Broadhurst, T., Bouwens, R., Silk, J. \& Rosati, P. 1999, ApJ, 515, L65

Benítez, N. 2000, ApJ, 536, 571

Benítez, N., et al. 2004, ApJS, 150, 1

Bertin, E., \& Arnouts, S. 1996, A\&AS, 117, 393

Bohlin, R. C., Lindler, D. J., \& Riess, A. 2005 NICMOS Instrument Science Report 2005-002 (Baltimore: STScI)

Bohlin, R. C., Riess, A., \& de Jong, R. 2006 NICMOS Instrument Science Report 2006-002 (Baltimore: STScI)

Bouwens, R. J., Illingworth, G. D., Blakeslee, J. P., \& Franx, M. 2005, ArXiv Astrophysics e-prints, arXiv:astro-ph/0509641

Bruzual, G., \& Charlot, S. 2003, MNRAS, 344, 1000

Bunker, A. J., Stanway, E. R., Ellis, R. S., \& McMahon, R. G. 2004, MNRAS, 355, 374

Casertano, S., et al. 2000, AJ, 120, 2747

Cimatti, A., et al. 2002, A\&A, 392, 395

Coleman, G. D., Wu, C.-C., \& Weedman, D. W. 1980, ApJS, 43, 393

Conselice, C. J., Bershady, M. A., \& Jangren, A. 2000, ApJ, 529, 886

Conselice, C. J., Bershady, M. A., Dickinson, M., \& Papovich, C. 2003, AJ, 126, 1183

Cowie, L. L., Barger, A. J., Hu, E. M., Capak, P., \& Songaila, A. 2004, AJ, 127, 3137

Croom, S. M., Warren, S. J., \& Glazebrook, K. 2001, MNRAS, 328, 150

Dickinson, M., et al. 2004, ApJ, 600, L99 
Fernández-Soto, A., Lanzetta, K. M., \& Yahil, A. 1999, ApJ, 513, 34 (FLY99)

Fernández-Soto, A., Lanzetta, K. M., Chen, H.-W., Pascarelle, S. M., \& Yahata, N. 2001, ApJS, 135, 41

Ford, H. C., et al. 2002, Bulletin of the American Astronomical Society, 34, 675

Fruchter, A. S., \& Hook, R. N. 2002, PASP, 114, 144

Giavalisco, M., et al. 2004, ApJ, 600, L93

Gilli, R., et al. 2003, ApJ, 592, 721

Gwyn, S. D. J., \& Hartwick, F. D. A. 1996, ApJ, 468, L77

Gwyn, S. D. J., \& Hartwick, F. D. A. 2005, AJ, 130, 1337

de Jong, R. S., et al. 2006, NICMOS Instrument Science Report 2006-001 (Baltimore: STScI)

de Jong, R. S., et al. 2006, 2005 HST Calibration Workshop (Baltimore: STScI)

de Jong, R. S. 2006, NICMOS Instrument Science Report 2006-003 (Baltimore: STScI)

Kinney, A. L., Calzetti, D., Bohlin, R. C., McQuade, K., Storchi-Bergmann, T., \& Schmitt, H. R. 1996, ApJ, 467, 38

Koo, D. C. 1985, AJ, 90, 418

Kauffmann, G., White, S. D. M., Heckman, T. M., Ménard, B., Brinchmann, J., Charlot, S., Tremonti, C., \& Brinkmann, J. 2004, MNRAS, 353, 713

Le Fèvre, O., et al. 2004, A\&A, 428, 1043

Mobasher, B., \& Riess, A. 2005 NICMOS Instrument Science Report 2005-003 (Baltimore: $\mathrm{STScI})$

Mobasher, B., et al. 2005, ApJ, 635, 832

Peng, C. Y., Ho, L. C., Impey, C. D., \& Rix, H.-W. 2002, AJ, 124, 266

Rix, H.-W., et al. 2004, ApJS, 152, 163

Sánchez, S. F., et al. 2004, ApJ, 614, 586

Schade, D., Lilly, S. J., Crampton, D., Hammer, F., Le Fevre, O., \& Tresse, L. 1995, ApJ, 451, L1 
Schlegel, D. J., Finkbeiner, D. P., \& Davis, M. 1998, ApJ, 500, 525

Sérsic, J. L. 1968, Atlas de Galaxias Australes (Córdoba, Argentina: Press of the Universidad Nacional)

Shen, S., Mo, H. J., White, S. D. M., Blanton, M. R., Kauffmann, G., Voges, W., Brinkmann, J., \& Csabai, I. 2003, MNRAS, 343, 978

Sirianni, M., et al. 2003, American Astronomical Society Meeting Abstracts, 202,

Stanway, E. R., Bunker, A. J., \& McMahon, R. G. 2003, MNRAS, 342, 439

Stanway, E. R., Bunker, A. J., McMahon, R. G., Ellis, R. S., Treu, T., \& McCarthy, P. J. 2004, ApJ, 607, 704

Stanway, E. R., McMahon, R. G., \& Bunker, A. J. 2005, MNRAS, 359, 1184

Steidel, C. C., \& Hamilton, D. 1992, AJ, 104, 941

Stetson, P. B. 1994, PASP, 106, 250

Stiavelli, M., Fall, S. M., \& Panagia, N. 2004, ApJ, 610, L1

Strolger, L.-G., et al. 2004, ApJ, 613, 200

Szokoly, G. P., et al. 2004, ApJS, 155, 271

Thompson, R. I., et al. 2005, ArXiv Astrophysics e-prints, arXiv:astro-ph/0503504

Thompson, R. I., et al. 2006, ApJ, submitted

Vanzella, E., et al. 2001, AJ, 122, 2190

Vanzella, E., et al. 2005, A\&A, 434, 53

Williams, R. E., et al. 1996, AJ, 112, 1335

Williams, R., et al. 1998, Bulletin of the American Astronomical Society, 30, 1366

Wirth, G. D., et al. 2004, AJ, 127, 3121

Wolf, C., et al. 2004, A\&A, 421, 913

Wolf, C., et al. 2005, ApJ, 630, 771

Yan, H., \& Windhorst, R. A. 2004, ApJ, 612, L93 
This preprint was prepared with the AAS LATEX macros v5.2. 


\section{A. PSF Matching}

We used the DAOPHOT II/ALLSTAR software package (Stetson 1994) to determine the $i^{\prime}$, $J$, \& $H$ PSFs in the reduced images provided by STScI (that is after drizzling but before any re-mapping). The brightest non-saturated stars in the $i^{\prime}$ image were sufficient for DAOPHOT to compute an average PSF. The $i^{\prime}$ PSF was accepted as the PSF for all WFC images, including the $B+V+i^{\prime}+z^{\prime}$ detection image $d$. (We verified that the PSFs for all five images are nearly identical. The PSF of the $z^{\prime}$ image is slightly worse, and we deal with it separately in $§ 3.3 .1$.)

Unfortunately these stars alone could not be used to determine the NIC3 PSFs. The NIC3 images of these stars are highly asymmetric (perhaps due to the image reduction). So we allowed DAOPHOT to average many much fainter "stars" to determine the NIC3 PSFs. Many of these objects are not stars at all; they are resolved in the WFC images as extended objects. But these objects are still narrow enough to appear as point sources to NIC3. Thus they are suitable for use in the NIC3 PSF determination.

Once the $i^{\prime}, J, \& H$ PSFs are determined, the $J \& H$ PSFs are re-mapped to the WFC frame (using IRAF's wregister, interp=spline3). (The re-mapping process is controlled to ensure that each re-mapped NIC3 PSF is centered on a pixel in the WFC frame.) Then IRAF's psfmatch is used to determine the kernels necessary to degrade the $i^{\prime}$-band PSF to match the re-mapped NIC3 PSFs. We use these psfmatching kernels to degrade $d$ (which has a PSF nearly identical to $i^{\prime}$ ) to the NIC3 PSFs, yielding images $d^{J} \& d^{H}$. Meanwhile, the NIC3 images are re-mapped to the WFC frame, yielding images $J^{A} \& H^{A}$ (see Fig. 3). Finally, by training identical apertures on a given galaxy in $d^{J} \& J^{A}$, we obtain a robust measure of the $d-J$ color for that galaxy. Similarly, we obtain the $d-H$ color. The $d-B$, $d-V, d-i^{\prime}, \& d-z^{\prime}$ colors are all obtained without the need for such PSF matching gymnastics, and we arrive at 6 colors referenced against the same image, $d$. (Again, the $z^{\prime}$ PSF correction is handled separately in $§ 3.3 .1$.

A much simpler PSF matching approach proves unreliable: instead of determining the PSFs explicitly, one could simply use IRAF's psfmatch to degrade $d$ directly to the PSF of the remapped NIC3 images $J^{A} \& H^{A}$. But psfmatch has a difficult time determining the $J$ \& $H$ PSFs from the re-mapped NIC3 images. Each star in the NIC3 images is pixelized and therefore slightly asymmetric. These asymmetries are greatly magnified by the re-mapping. psfmatch returns highly distorted kernels, with very significant effects on the magnitudes of faint objects. The asymmetries can be averaged out much more effectively before re-mapping. 


\section{B. Morphology Simulations}

To determine the limits of reliability of the extracted galaxy profile parameters we have performed a set of simulations. Simulated galaxies were created using galfit assuming a Sérsic profile and fixing all the model parameters to a set of input values. Particular care was taken to resample the noise histogram and noise pattern seen in the UDF ACS dithered images, as described in Sánchez et al. (2004). Our first simulation reproduces the UDF, with all galaxy positions and morphological parameters as derived from the UDF $i^{\prime}$ band image. Our second simulation retains the galaxy positions (preserving the observed clumpiness) but shuffles the galaxies among them. Also, random scatter is added to the observed morphological parameters. Each galaxy's effective radius $R_{e}$, semiaxis ratio $a / b$, and Sérsic index $n$ were changed randomly within $20 \%$ of their original values. Magnitude $i^{\prime}$ was altered within \pm 0.25 mags, and the position angle $\theta$ was randomly rotated. Our third and final simulation bears little resemblance to the UDF. The observed catalog is scrapped in favor of new galaxies which are distributed homogeneously throughout the image. Galaxy parameters are chosen randomly with flat distributions: $21<i^{\prime}<31,0.2<b / a<0.8,0.2<$ $n<8, \quad 0<\theta<2 \pi$. The effective radii follow a relation $\log _{10}\left(R_{e}\right) \sim 5.62-0.18 \times i^{\prime} \pm 0.5$, where $R_{e}$ is measured in pixels, and a random scatter of 0.5 has been added to the logarithm. Due to the flat magnitude distribution, this last simulation yields many more large and bright galaxies. To avoid overcrowding the field, we create only 5,000 galaxies.

The simulated images are analyzed by running SExtractor and galfit as was done for the UDF $i^{\prime}$-band image. Output galaxies are matched to input galaxies by object position (centroids within $R_{e} / 4$ and within 10 pixels). We find agreement between input and output parameters, with no appreciable biases and the following scatters: $\Delta i^{\prime}=0.35, \Delta R_{e} / R_{e} \sim$ $0.2, \Delta(b / a)=0.17, \Delta n=1.6$ (see Fig. 32).

As mentioned in $\S 3.5$, we adopt $n=2.5$ as the dividing line between late $(n<2.5)$ and early $(n>2.5)$ type galaxies. But our ability to classify galaxies as late or early type depends on the galfit uncertainty $\sigma n / n$. (Note that galfit's $\sigma n$ is not defined as a typical RMS uncertainty.) From our simulations, we find we retrieve $n$ to within $\Delta n=1$ for bright $\left(i^{\prime}<26\right)$ and large $\left(R_{e}>10\right.$ pixels $)$ galaxies and to within $\Delta n=2$ for faint $\left(i^{\prime}>28\right)$ and small ( $R_{e}<3$ pixels) galaxies (Fig. 33). If we assume that $n=2.5$ is a perfect discriminator between late and early type galaxies, then we can make some simple predictions about our ability to classify galaxies. For example, if we select only those galaxies with $\sigma n / n<1$, then $80-95 \%$ of them will be correctly classified as late $(n<2.5)$ or early $(n>2.5)$ (Figs. 34a \& 34b). (Fig. 34c is based on simulation \#3 which contains too many bright, and thus easily classifiable, galaxies.) This $\sigma n / n<1$ cut only discards $\sim 8 \%$ of the catalog. Thus we recommend it as a good compromise between selecting the maximum number of objects and 

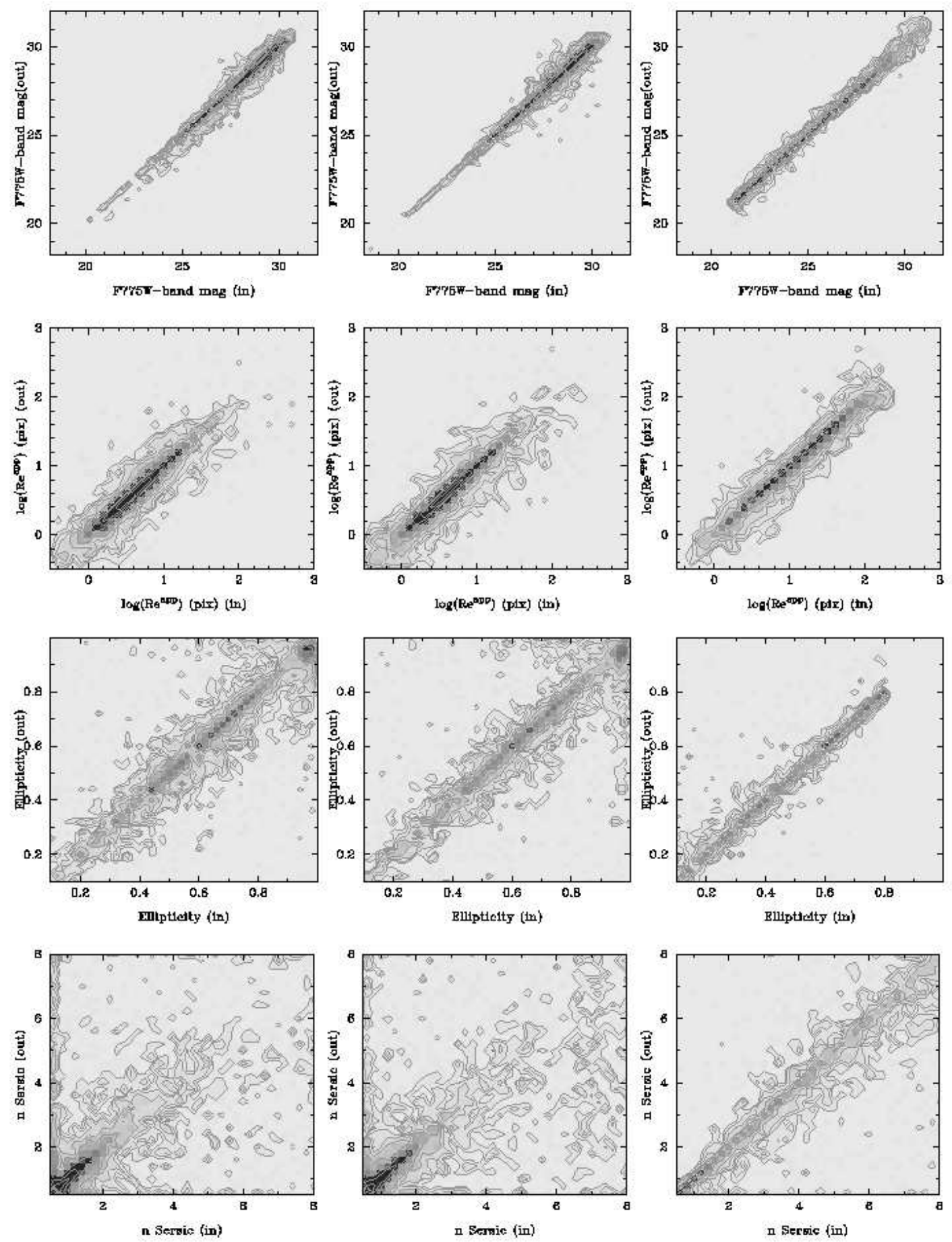

Fig. 32.- Distribution of the output parameters as a function of the input parameters for the three simulations. The panels include from top to bottom the distributions of the $i^{\prime}$-band magnitudes, the effective radii $R_{e}$, the ellipticities and the Sérsic indices $n$, and from left to right, the results from the first, second and third simulations. 
selecting a reliable sample.

Figure 35 compares the UDF galaxy asymmetries with those from simulation \#1. The simulated galaxies are intrinsically symmetric, so any observed asymmetry is due to noise. Asymmetry measurements of real galaxies that fall below those typical of simulated galaxies of similar magnitude should not be considered reliable. 

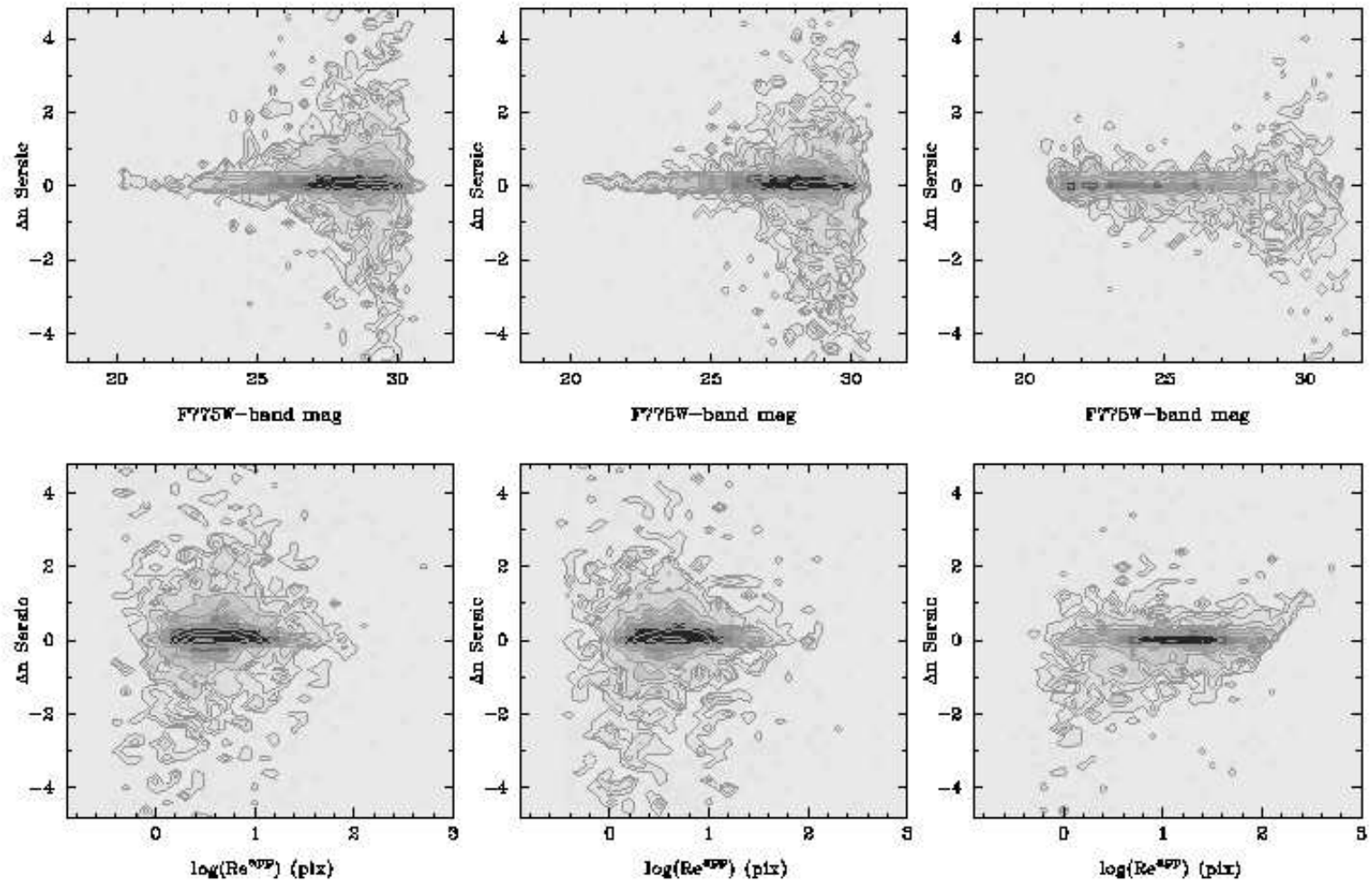

Fig. 33. - Sérsic index errors $\Delta n=n_{\text {out }}-n_{\text {in }}$ as a function of the $i^{\prime}$-band magnitudes (top row) and as a function of the effective radii $R_{e}$ (bottom row) for the three simulations (left to right).
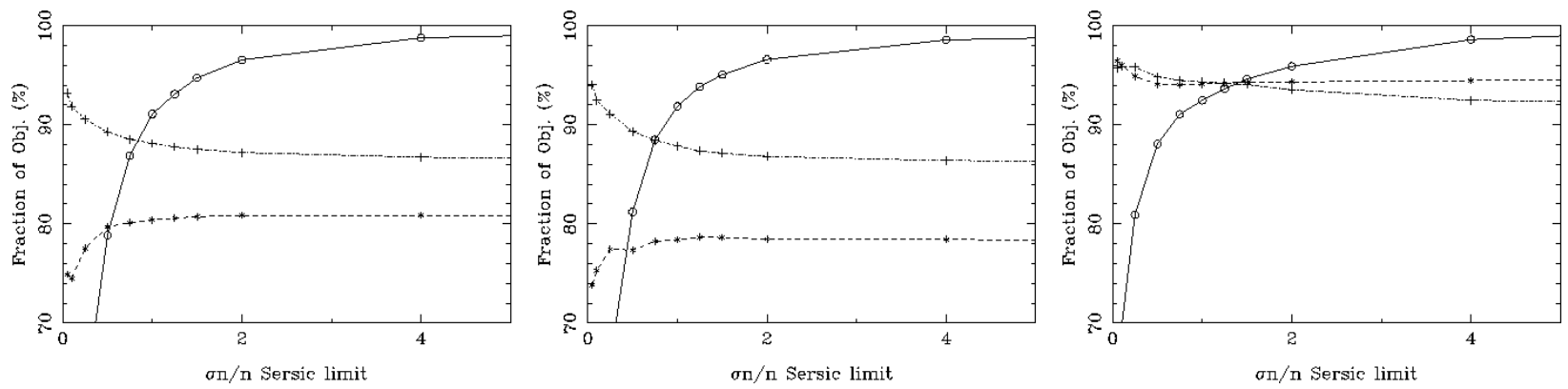

Fig. 34.- Effects of pruning the catalogs resulting from the three simulations (left to right) based on Sérsic index uncertainty (e.g., $\sigma n / n<1$ ). The solid black lines (with circles) show the total fraction of galaxies spared by the cuts. The dot-dashed green (stars) and dashed red (crosses) lines show the fraction of well classified late and early type galaxies in the pruned catalogs (assuming that $n=2.5$ is a perfect discriminator). 


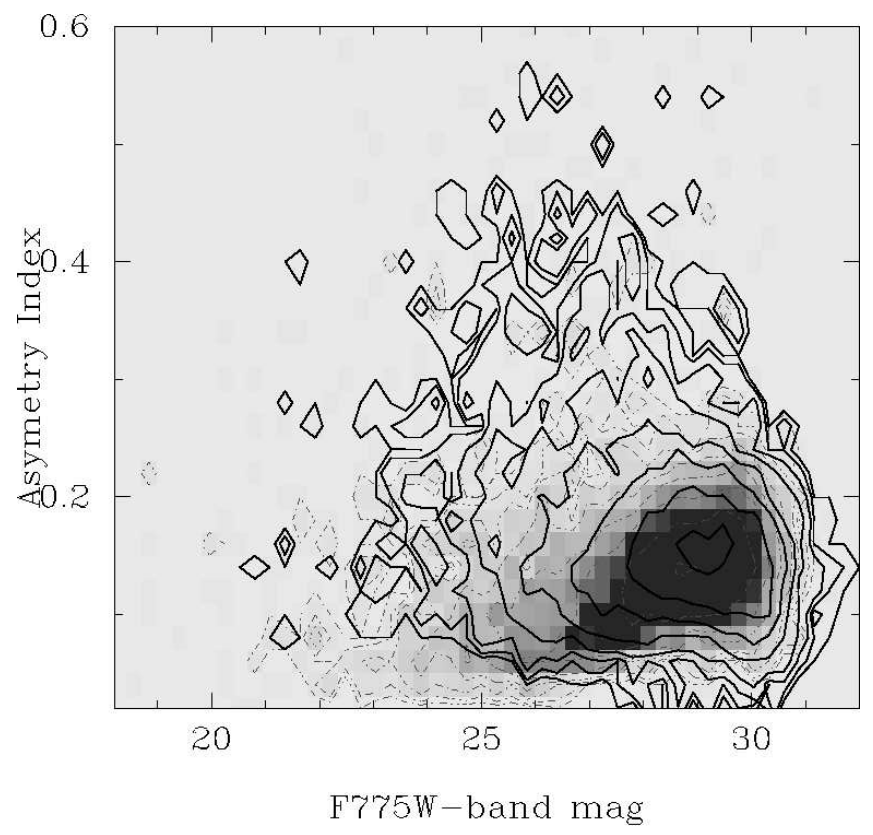

Fig. 35.- Distribution of the asymmetry indices as a function of $i^{\prime}$-band magnitude for the UDF galaxies (continuous contours) overlaid on top of the distribution for galaxies in simulation \#1 (greyscale and discontinuous contours). 